\title{
Analytische und numerische Untersuchung von direkten und inversen Randwertproblemen in Gebieten mit Ecken mittels Integralgleichungsmethoden
}

\author{
Dissertation \\ zur Erlangung des Doktorgrades \\ der Mathematisch-Naturwissenschaftlichen Fakultäten \\ der Georg-August-Universität zu Göttingen
}

\author{
vorgelegt von \\ ANDREAS VOGT \\ aus \\ Leinefelde
}


D7

Referent:

Korreferent:

Tag der mündlichen Prüfung:
Prof. Dr. R. Kreß

Prof. Dr. G. Lube

31. Oktober 2001 


\section{Inhaltsverzeichnis}

\begin{tabular}{lll}
\hline & Einleitung & 3 \\
\hline
\end{tabular}

$\begin{array}{llr}2 & \text { Grundlagen } & 8\end{array}$

$2.1 \quad$ Zu den Grundlösungen von Laplace- und Helmholtzgleichung . . . . . . . . 8

2.1 .1 Eigenschaften der Hankelfunktionen . . . . . . . . . . . . . . . . . . 9 9

2.2 Definition und Rechenregeln zur Fréchet-Ableitung . . . . . . . . . . . . . 10

2.3 Funktionalanalytische Grundlagen . . . . . . . . . . . . . . . . . . . . . . . 12

$\begin{array}{lll}3 & \text { Eine singuläre Integralgleichung } & 14\end{array}$

3.1 Zur Existenz und Eindeutigkeit . . . . . . . . . . . . . . . . . . . . 14

3.2 Die Substitutionsmethode . . . . . . . . . . . . . . . . . . . . . . 16

3.3 Ein Nyström - Verfahren . . . . . . . . . . . . . . . . . . . . . . . . . . . . 21

4 Das direkte Streuproblem 24

4.1 Randwertprobleme zur Helmholtzgleichung . . . . . . . . . . . . . . . . . . 25

4.2 Zur Eindeutigkeit beim äußeren Dirichlet-Problem . . . . . . . . . . . . . . 25

4.3 Potenziale und Sprungbeziehungen . . . . . . . . . . . . . . . . . . . . . 31

4.4 Zur Existenz beim direkten Dirichletproblem . . . . . . . . . . . . . . . . 33

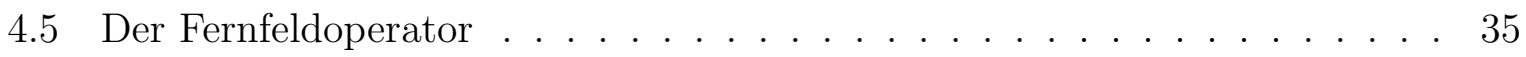

4.6 Numerische Ergebnisse $\ldots \ldots \ldots$. . . . . . . . . . . . . . . . . 37 
5 Das inverse Streuproblem $\quad 45$

5.1 Eindeutigkeit beim inversen Streuproblem . . . . . . . . . . . . . . . . . . 45

5.2 Ableitung der Randintegraloperatoren . . . . . . . . . . . . . . . . . 47

5.3 Die Fréchet - Ableitung des Fernfeldoperators . . . . . . . . . . . . . . . . 63

$6 \quad$ Numerische Ergebnisse beim inversen Streuproblem 69

6.1 Newtonverfahren und Regularisierung . . . . . . . . . . . . . . . . . . . . 69

6.2 Modifizierung im Tikhonov-Funktional . . . . . . . . . . . . . . . . . . . . 73

6.3 Die rechte Seite der Fréchet - Ableitung . . . . . . . . . . . . . . . . . . . 75

6.4 Rekonstruktionen mit glatten Kurven . . . . . . . . . . . . . . . . . . . . . 78

6.5 Rekonstruktionen mit nicht-glatten Kurven . . . . . . . . . . . . . . . . . 83

6.6 Eingeschränkter Messbereich . . . . . . . . . . . . . . . . . . . . . . . 86

7 Ein spezielles Impedanzrandwertproblem 90

7.1 Formulierung des Impedanzrandwertproblems . . . . . . . . . . . . . . . . 90

7.2 Existenz und Eindeutigkeit beim direkten Impedanzrandwertpr. . . . . . . 92

7.3 Der hypersinguläre Operator $T_{0} \ldots \ldots \ldots$

7.4 Numerische Ergebnisse . . . . . . . . . . . . . . . . . . . . . . . 102

8 Das inverse Impedanzrandwertproblem 108

8.1 Eindeutigkeit beim inversen Impedanzrandwertproblem . . . . . . . . . . . 108

8.2 Die Fréchet - Ableitung des Randwerteoperators . . . . . . . . . . . . . . . 110

8.3 Numerische Ergebnisse . . . . . . . . . . . . . . . . . . . . . . . . . . . . . 117

$\begin{array}{ll}\text { Literaturverzeichnis } & 123\end{array}$ 


\section{Kapitel 1}

\section{Einleitung}

Zwei Probleme heißen zueinander invers, wenn die Formulierung des einen die Formulierung des anderen erfordert und umgekehrt. Zunächst ist es unwichtig, welches der beiden Probleme als direktes und welches als inverses Problem bezeichnet wird. In der Regel wird das historisch ältere und besser verstandene Problem als direktes Problem bezeichnet. Für eine Reihe von physikalisch motivierten Beispielen sei auf das Buch von Groetsch [17] verwiesen.

Zahlreiche physikalische Fragestellungen führen auf partielle Differenzialgleichungen, deren Lösung zusätzliche Bedingungen auf dem Rand eines Gebietes erfüllen muss. Stellvertretend seien an dieser Stelle die Streuung von akustischen und elektromagnetischen Wellen, die Elastizitätstheorie und Strömungsprobleme erwähnt.

Für partielle Differenzialgleichungen mit konstanten Koeffizienten sind Integralgleichungsmethoden ein wesentliches Hilfsmittel zur Untersuchung dieser direkten Randwertprobleme.

Bei den zugehörigen inversen Problemen sind aus gemessenen Daten bestimmte physikalische Parameter, wie etwa der Rand eines Gebietes, die Wellenzahl oder die Leitfähigkeit, zu rekonstruieren. Während in der Regel das direkte Problem linear und wohlgestellt ist, ist das inverse Problem nichtlinear und schlecht gestellt im Sinne von Hadamard. Für eine numerische Behandlung sind neben einer geeigneten Linearisierung, die zum Begriff der Fréchet-Differenzierbarkeit führt, daher auch Regularisierungstechniken erforderlich.

In dieser Arbeit habe ich stellvertretend zwei Beispiele betrachtet, zum einen das direkte und inverse Dirichletproblem zur Helmholtzgleichung in Gebieten mit Ecken und zum anderen ein spezielles Impedanzrandwertproblem, bei dem in natürlicher Weise (durch Korrosionseffekte) Ecken auftreten. 
Die mathematische Modellierung der Streuung von zeitharmonischen Wellen an Hindernissen führt zu äußeren Randwertproblemen für die Helmholtzgleichung

$$
\triangle u+k^{2} u=0
$$

Je nachdem, ob der Druck oder die Geschwindigkeit der Welle beim Auftreffen auf das Hindernis vorgeschrieben werden, führt dies zu einem Dirichletproblem (schallweiches Hindernis) oder zum Neumannproblem (schallhartes Hindernis). Falls der Rand des Gebietes hinreichend glatt ist, führen Potenzialansätze auf Integralgleichungen zweiter Art mit einem kompakten Operator. Die auf diese Weise erzielten Existenz-, Eindeutigkeitsund Konvergenzresultate finden sich bei Leis [40] im Fall des Dirichlet-Problems und bei Kussmaul [38] für das Neumann-Problem im $\mathbb{R}^{2}$.

Falls das Gebiet Ecken aufweist, tritt eine zusätzliche Problematik auf. Die resultierende Randintegralgleichung hat nicht mehr die Form Identität plus kompakter Operator (siehe Willers [57, Seiten 90-94].) Die Riesz-Theorie ist trotzdem anwendbar, denn der Operator ist Summe aus einem kompakten Operator, der Identität und einem Operator mit Norm kleiner eins. Die Konvergenz- und Fehleranalysis für ein Nyström-Verfahren zur numerischen Behandlung der Integralgleichung, die sich bei der Laplace-Gleichung ergibt und dessen Kern Singularitäten in den Ecken aufweist, geht zurück auf Kreß [28]. Der bei der Helmholtzgleichung zusätzlich auftretende logarithmische Anteil ist in der Arbeit von Kümpel [39.] berücksichtigt worden.

Weitere ähnlich gelagerte Beispiele ergeben sich bei der Reflexion an lokal gestörten Ebenen (siehe Willers [57], Hartke [1.9] oder Boeckh [2]) oder der Streuung am Kurvenbogen (siehe Kreß [3]] oder Mönch [44]).

Für Beispiele, wie mit Integralgleichungen erster Art ähnliche Konvergenzresultate erzielt werden, sei verwiesen auf Mönch [43], Chapko-Kreß [5], Kreß-Sloan [29] und die Arbeiten von Elschner [12], [13].

Beim direkten Problem ist bei bekanntem Gebiet im $\mathbb{R}^{2}$ das gestreute Feld $u^{s}$ zu ermitteln. Die Sommerfeldsche Ausstrahlungsbedingung impliziert für $u^{s}$ das asymptotische Verhalten

$$
u^{s}(x)=\frac{e^{i k|x|}}{\sqrt{|x|}}\left\{u_{\infty}(\hat{x})+O\left(\frac{1}{|x|}\right)\right\},|x| \rightarrow \infty,
$$

gleichmäßig für alle Richtungen $\hat{x}=x /|x|$. Der Amplitudenfaktor $u_{\infty}$ heißt Fernfeld von $u$. Vom funktionalanalytischem Standpunkt bedeutet dies, den sogenannten Fernfeldoperator

$$
\mathcal{F}: C_{2 \pi}(\mathbb{R}) \cap C^{2, \alpha}[0,2 \pi] \rightarrow L^{2}(\Omega), z \mapsto u_{\infty}
$$


der den Rand des Streugebiets auf das Fernfeld abbildet, auszuwerten. Hierbei ist $\Omega$ gegeben durch $\Omega=\left\{x \in \mathbb{R}^{2}|| x \mid=1\right\}$.

Beim inversen Problem besteht die Aufgabe darin, aus der Kenntnis von Fernfelddaten für eine oder mehrere einfallende ebene Wellen, den Rand des Gebietes zu rekonstruieren. Demnach ist $\mathcal{F}$ zu invertieren. Da der Fernfeldoperator nichtlinear und kompakt ist, wird für ein numerisches Verfahren sowohl eine geeignete Linearisierung benötigt, als auch eine Regularisierung erforderlich.

Neben dem akustischen Streuproblem habe ich ein spezielles Impedanzrandwertproblem betrachtet. Durch Vergraben eines metallischen Gegenstandes (mit konstanter Leitfähigkeit) kommt es zu Korrosionseffekten auf dem in der Erde befindlichen Teil der Oberfläche und daher zur Ausbildung von Ecken. Durch Einprägen von Strom auf einem nicht korrodierten Oberflächenstück kommt es zu einer Spannungsverteilung im Körper.

Das direkte Problem besteht darin, bei bekannter Oberfläche den Strom auf einem nicht korrodierten Teil zu ermitteln. Mathematisch modelliert bedeutet dies, die Neumannrandwerte der Lösung eines inneren Dirichletproblems zur Laplacegleichung zu ermitteln.

Beim inversen Problem besteht die Aufgabe darin, aus der Kenntnis der Dirichlet- und Neumannwerte auf einem Teilstück, die unbekannte Oberfläche zu ermitteln. Dabei habe ich mich wiederum auf den zweidimensionalen Fall beschränkt.

Für weitere Beispiele von inversen Problemen, die mit newtonartigen Verfahren gelöst wurden, verweise ich stellvertretend auf Gerlach [14], Hohage-Schormann [22], Kreß [30], [31], [32], Kreß-Tran [37], Mönch [44], Potthast [45], [46], [47] und Schormann [5]], [52].

Nachfolgend erläutere ich den Aufbau der Arbeit.

Im Kapitel 2 habe ich die Grundlösungen zur Laplace- und Helmholtzgleichung angegeben. Aufgrund der komplizierteren Struktur der Grundlösung im $\mathbb{R}^{2}$, die durch die Hankelfunktion $H_{0}^{(1)}$ gegeben ist, habe ich für $H_{n}^{(1,2)}$ die später benötigten Eigenschaften im Abschnitt 2.1 separat aufgeführt. Der Abschnitt 2.2 ist der Fréchet-Ableitung und deren Eigenschaften gewidmet.

Im Kapitel 3 habe ich eine singuläre Integralgleichung der Form

$$
\begin{gathered}
\varphi(t)-\int_{0}^{2 \pi}\left\{K_{A}(t, \tau)+K_{B}(t, \tau)+K_{C}(t, \tau) \ln \left(4 \sin ^{2} \frac{t-\tau}{2}\right)\right\}[\varphi(\tau)-\varphi(0)] d \tau \\
+\gamma(t) \varphi(0)=f(t), \quad 0 \leq t \leq 2 \pi
\end{gathered}
$$

betrachtet, bei denen die auftretenden Kerne stetig oder schwach singulär sind oder in den Ecken integrierbare Singularitäten aufweisen. Im Abschnitt 3.1 zeige ich, dass für 
EINLEITUNG

diese Gleichung zweiter Art die Riesz-Theorie angewendet werden kann. Im Abschnitt 3.2 gehe ich auf die numerische Behandlung von eindimensionalen Integralen über kompakten Mengen, deren Integranden Endpunktsingularitäten besitzen, ein. Die Idee besteht darin, Singularitäten zunächst durch geeignete Substitutionen zu beseitigen und anschließend die Trapezregel anzuwenden. In [55] habe ich bereits verschiedene Substitutionen vorgestellt und miteinander verglichen, sodass ich in der vorliegenden Arbeit stillschweigend darauf zurückgreifen kann. Im Abschnitt 3.3 gehe ich auf das Nyström - Verfahren zur approximativen Lösung der singulären Integralgleichung ein. Abschließend habe ich das Konvergenzresultat, dessen ausführlicher Beweis bei Kümpel [39] nachzulesen ist, angegeben und den Beweisgang grob skizziert.

Im Kapitel 4 ist das direkte Streuproblem von mir behandelt worden. Nach der Formulierung des äußeren Dirichlet- und inneren Neumannproblems werden im Abschnitt 4.2 die Eindeutigkeitssätze formuliert und bewiesen. Mit den im Abschnitt 4.3 erklärten Potenzialen und deren Sprungbeziehungen zeige ich im Abschnitt 4.4, dass das äußere Dirichletproblem (für Gebiete mit genau einer Ecke) eine Lösung besitzt. Dabei wird gezeigt, dass die unbekannte Dichte eine injektive Integralgleichung vom Typ (1.1) löst. Im Abschnitt 4.5 ist das Fernfeld einer ausstrahlenden Lösung der Helmholtzgleichung erklärt. Im letzten Abschnitt 4.6 habe ich numerische Ergebnisse für verschiedene Gebiete mit verschiedenen Substitutionen angeführt.

Das Kapitel 5 habe ich dem inversen Streuproblem gewidmet. Im Abschnitt 5.1 zeige ich, dass bei Übereinstimmung der Fernfelder für alle Einfallsrichtungen $d \in \Omega$ die Streugebiete gleich sind, also die Injektivität des Fernfeldoperators $\mathcal{F}$. In den beiden nächsten Abschnitten wird die Fréchet-Differenzierbarkeit der Abbildung $z \mapsto \mathcal{F}(z)$ nachgewiesen und die Ableitung $\mathcal{F}^{\prime}(z, h)$ durch ein Randwertproblem charakterisiert. Satz 5.13 ist damit das Hauptresultat des Kapitels 5 .

Im Kapitel 6 habe ich das numerische Verfahren zur Lösung des inversen Streuproblems erläutert und Beispiele von Rekonstruktionen angegeben. Den Darstellungen ist zu entnehmen, dass es sich lohnt, Gebiete mit Ecken nicht nur mit glatten Ansatzfunktionen zu rekonstruieren, sondern nicht-glatte Anteile zur besseren Rekonstruktion zu berücksichtigen. Ein Konvergenzbeweis für das Newtonverfahren zur Lösung inverser Streuprobleme ist bisher in der Literatur nicht bekannt. Zur Problematik der Konvergenz von newtonartigen Verfahren gibt es zwar allgemeine funktionalanalytische Sätze (siehe etwa [1T]), deren Voraussetzungen jedoch bei Streuproblemen im allgemeinen nicht erfüllt sind.

Die Kapitel 7 und 8 widmen sich dem Impedanzproblem. Zunächst habe ich für das direkte Problem Existenz und Eindeutigkeit einer Lösung gezeigt. Bei der numerischen Behandlung der resultierenden Randintegralgleichung für die unbekannte Dichte tritt auf- 
grund von zwei Ecken eine zusätzliche Problematik auf. Im Abschnitt 7.3 gehe ich auf die Behandlung des hypersingulären Operators $T_{0}$ ein und im Abschnitt 7.4 habe ich die numerischen Ergebnisse angeführt.

Anstelle des Fernfeldoperators tritt hier der Randwerteoperator $\mathcal{F}$, der bei einem festen Dirichletwert $f$ hinreichend glatte Randkurven $z$ abbildet auf die Neumannwerte $\frac{\partial u}{\partial \nu}$ der Lösung $u$ des inneren Dirichletproblems.

Im Kapitel 8 stelle ich dar, wie sich, wiederum mit einem regularisierten Newtonverfahren, der unbekannte Rand aus Messdaten rekonstruieren lässt. Dazu zeige ich zunächst im Abschnitt 8.1 die Injektivität des Randwerteoperators $\mathcal{F}$ und im Abschnitt 8.2 die FréchetDifferenzierbarkeit von $\mathcal{F}$ bei geeigneten Funktionenräumen. Anschließend charakterisiere ich die Ableitung durch ein Randwertproblem. Dabei stellt Satz 8.7 das Analogon zu Satz 5.13 dar. Die numerischen Ergebnisse habe ich im Abschnitt 8.3 angeführt.

Herrn Prof. Dr. Rainer Kreß danke ich recht herzlich für die Anregung zur Beschäftigung mit dieser Thematik und seine wertvollen Ratschläge bei der Anfertigung dieser Arbeit. Herrn Prof. Dr. Gert Lube danke ich für die Übernahme des Korreferats. 


\section{Kapitel 2}

\section{Grundlagen}

\subsection{Zu den Grundlösungen von Laplace- und Helm- holtzgleichung}

Für die in den nachfolgenden Kapiteln behandelten Randwertprobleme werden die Grundlösungen zur Laplace- und Helmholtzgleichung benötigt. Diese lauten

$$
\Phi_{0}(x, y)=\left\{\begin{array}{cl}
\frac{1}{2 \pi} \ln \frac{1}{|x-y|}: m=2 \\
\frac{1}{4 \pi} \frac{1}{|x-y|} \quad: m=3
\end{array} \quad x, y \in \mathbb{R}^{m}, x \neq y\right.
$$

im Fall der Laplacegleichung und

$$
\Phi_{k}(x, y)=\left\{\begin{array}{cl}
\frac{i}{4} H_{0}^{(1)}(k|x-y|) & : m=2 \\
\frac{1}{4 \pi} \frac{e^{i k|x-y|}}{|x-y|} & : m=3
\end{array} \quad x, y \in \mathbb{R}^{m}, x \neq y\right.
$$

für die Helmholtzgleichung $\triangle u+k^{2} u=0$. In dieser Arbeit beschränken wir uns weitestgehend auf den zweidimensionalen Fall. Insbesondere bei den numerischen Verfahren werden Eigenschaften der Hankelfunktionen benötigt, die daher im nächsten Abschnitt zusammengetragen werden. 


\subsubsection{Eigenschaften der Hankelfunktionen}

In Polarkoordinaten erhält die Gleichung $\triangle u+k^{2} u=0$ die Form

$$
\frac{\partial^{2} u}{\partial r^{2}}+\frac{1}{r} \frac{\partial u}{\partial r}+\frac{\partial^{2} u}{\partial \varphi^{2}}+k^{2} u=0 .
$$

Wir suchen im $\mathbb{R}^{2}$ Lösungen der Helmholtzgleichung der Form

$$
u(x)=f(k r) e^{ \pm i n \varphi} .
$$

Dies führt auf die Besselsche Differenzialgleichung

$$
t^{2} f^{\prime \prime}(t)+t f^{\prime}(t)+\left[t^{2}-n^{2}\right] f(t)=0, \quad n \in \mathbb{N}_{0} .
$$

Offensichtlich ist die Besselfunktion

$$
J_{n}(t):=\sum_{p=0}^{\infty} \frac{(-1)^{p}}{p !(n+p) !}\left(\frac{t}{2}\right)^{n+2 p}, \quad n \in \mathbb{N}_{0},
$$

Lösung von (2.1) und analytisch für $t \in \mathbb{C}$. Eine zweite linear unabhängige Lösung wird durch die Neumannfunktion

$$
\begin{aligned}
Y_{n}(t):= & \frac{2}{\pi}\left\{\ln \frac{t}{2}+C_{E}\right\} J_{n}(t)-\frac{1}{\pi} \sum_{p=0}^{n-1} \frac{(n-1-p) !}{p !}\left(\frac{2}{t}\right)^{n-2 p} \\
& -\frac{1}{\pi} \sum_{p=0}^{\infty} \frac{(-1)^{p}}{p !(n+p) !}\left(\frac{t}{2}\right)^{n+2 p}\{\psi(p+n)+\psi(p)\}, n \in \mathbb{N}_{0}
\end{aligned}
$$

mit der Funktion

$$
\psi(0):=0, \quad \psi(p):=\sum_{m=1}^{p} \frac{1}{m}, p \in \mathbb{N}
$$

und der Eulerkonstanten

$$
C_{E}:=\lim _{p \rightarrow \infty}\left\{\sum_{m=1}^{p} \frac{1}{m}-\ln p\right\},
$$

gegeben. Offenbar sind die $Y_{n}$ für $t>0$ analytisch. Die Linearkombinationen

$$
H_{n}^{(1,2)}:=J_{n} \pm i Y_{n}
$$


heißen Hankelfunktionen erster und zweiter Art der Ordnung $n$. Für das asymptotische Verhalten mit $t \rightarrow \infty$ findet man

$$
\begin{aligned}
& H_{n}^{(1,2)}(t)=\sqrt{\frac{2}{\pi t}} e^{ \pm i\left(t-\frac{n \pi}{2}-\frac{\pi}{4}\right)}\left\{1+O\left(\frac{1}{t}\right)\right\}, \quad t \rightarrow \infty, \\
& H_{n}^{(1,2)^{\prime}}(t)=\sqrt{\frac{2}{\pi t}} e^{ \pm i\left(t-\frac{n \pi}{2}+\frac{\pi}{4}\right)}\left\{1+O\left(\frac{1}{t}\right)\right\}, \quad t \rightarrow \infty .
\end{aligned}
$$

Den Potenzreihen (2.2) und (2.3) ist für die Fundamentallösung

$$
\Phi_{k}(x, y):=\frac{i}{4} H_{0}^{(1)}(k|x-y|), \quad x \neq y
$$

der Helmholtzgleichung im $\mathbb{R}^{2}$ das asymptotische Verhalten

$$
\Phi_{k}(x, y)=\frac{1}{2 \pi} \ln \frac{1}{|x-y|}+\frac{i}{4}-\frac{1}{2 \pi} \ln \frac{k}{2}-\frac{C_{E}}{2 \pi}+O\left(|x-y|^{2} \ln \frac{1}{|x-y|}\right)
$$

für $|x-y| \rightarrow 0$ abzulesen.

\subsection{Definition und Rechenregeln zur Fréchet-Ableitung}

Für normierte Räume $X$ und $Y$ wird unter dem Symbol $\mathcal{L}(X, Y)$ die Menge der linearen und beschränkten Abbildungen von $X$ nach $Y$ verstanden. Die Menge $\mathcal{L}(X, Y)$ wird mit den üblichen punktweise erklärten Operationen zum linearen Raum, auf dem durch

$$
\|F\|_{\mathcal{L}(X, Y)}:=\sup _{\|x\|_{X}=1}\|F(x)\|_{Y}, F \in \mathcal{L}(X, Y)
$$

eine Norm gegeben ist. Mit $Y$ ist auch $\mathcal{L}(X, Y)$ ein Banachraum.

\section{Definition 2.1 (Definition der Fréchet-Ableitung)}

Seien $X, Y$ normierte Räume und $U \subset X$ offen. $F: U \rightarrow Y$ heißt Fréchet-differenzierbar in $x \in U$, wenn eine Abbildung $F^{\prime}(x) \in \mathcal{L}(X, Y)$ existiert, sodass gilt

$$
\left\|F(x+h)-F(x)-F^{\prime}(x) h\right\|_{Y}=o\left(\|h\|_{X}\right), h \rightarrow 0
$$


Zur Vereinfachung der Darstellung wird statt $F^{\prime}(x) h$ lediglich $F^{\prime}(x, h)$ geschrieben. Ist $F$ in jedem $x \in U$ Fréchet-differenzierbar, dann heißt die Abbildung

$$
\begin{aligned}
F^{\prime}: U & \longrightarrow \mathcal{L}(X, Y) \\
x & \longmapsto F^{\prime}(x, \cdot)
\end{aligned}
$$

die Fréchet-Ableitung von F.

\section{Satz 2.2 (Kettenregel)}

Seien $X, Y, Z$ normierte Räume und $X^{\prime} \subset X, Y^{\prime} \subset Y$ offen. Weiter seien $G: X^{\prime} \rightarrow Y^{\prime}$ in $x \in X^{\prime}$ und $F: Y^{\prime} \rightarrow Z$ in $G(x) \in Y^{\prime}$ Fréchet-differenzierbar. Dann ist $F \circ G: X^{\prime} \rightarrow Z$ in $x \in X^{\prime}$ Fréchet-differenzierbar, und es gilt

$$
(F \circ G)^{\prime}(x, h)=F^{\prime}\left(G(x), G^{\prime}(x, h)\right) \text { für alle } h \in X \text {. }
$$

\section{Satz 2.3 (Produktregel)}

Seien $U, X_{1}, X_{2}, Y$ normierte Räume, $U^{\prime} \subset U$ offen und

$$
b: X_{1} \times X_{2} \longrightarrow Y,\left(x_{1}, x_{2}\right) \longmapsto b\left(x_{1}, x_{2}\right)
$$

eine bilineare, beschränkte Abbildung. Desweiteren seien $F_{1}: U^{\prime} \rightarrow X_{1}$ und $F_{2}: U^{\prime} \rightarrow X_{2}$ Fréchet-differenzierbare Abbildungen. Dann ist

$$
\begin{aligned}
b\left(F_{1}, F_{2}\right): U^{\prime} & \rightarrow Y \\
u & \mapsto b\left(F_{1}(u), F_{2}(u)\right)
\end{aligned}
$$

Fréchet-differenzierbar und besitzt die Ableitung

$$
b\left(F_{1}, F_{2}\right)^{\prime}(u, h)=b\left(F_{1}^{\prime}(u, h), F_{2}(u)\right)+b\left(F_{1}(u), F_{2}^{\prime}(u, h)\right), u \in U^{\prime}, h \in U .
$$

Beispiel 2.4 Seien $X_{2}, Y$ normierte Räume, $X_{1}=\mathcal{L}\left(X_{2}, Y\right)$ und

$$
b: \mathcal{L}\left(X_{2}, Y\right) \times X_{2} \rightarrow Y,\left(A, x_{2}\right) \mapsto A\left(x_{2}\right)
$$

Die Bilinearform entspricht also der Anwendung eines Operators auf ein Element des Definitionsbereichs. Die Ableitung von $u \mapsto A(u)\left(x_{2}(u)\right)$ lautet demnach $h \mapsto A^{\prime}(u, h)\left(x_{2}(u)\right)+$ $A(u)\left(x_{2}^{\prime}(u, h)\right)$. 
Satz 2.5 (Quotientenregel) Seien $X$ normierter Raum, $Y$ Banachraum, $U \subset X$ offen. Weiterhin sei die Abbildung $F: U \rightarrow \mathcal{L}(Y, Y)$ Fréchet-differenzierbar in $\varphi_{0} \in U$ und $F(\varphi) \in \mathcal{L}(Y, Y)$ beschränkt invertierbar für alle $\varphi \in Y$. Dann ist

$$
G: U \rightarrow \mathcal{L}(Y, Y), \varphi \mapsto(F(\varphi))^{-1}
$$

Fréchet-differenzierbar in $\varphi_{0}$ mit der Ableitung $G^{\prime}\left(\varphi_{0}, \cdot\right) \in \mathcal{L}(X, \mathcal{L}(Y, Y))$,

$$
G^{\prime}\left(\varphi_{0}, h\right)=-G\left(\varphi_{0}\right) F^{\prime}\left(\varphi_{0}, h\right) G\left(\varphi_{0}\right)
$$

Definition 2.6 (Ableitungen höherer Ordnung) Seien X, Y normierte Räume, $U \subset X$ offen und $F: U \rightarrow Y$ ein Fréchet-differenzierbarer Operator. Dann heißt $F$ an der Stelle $\varphi_{0} \in X$ zweimal Fréchet-differenzierbar, wenn die Abbildung $F^{\prime}: U \rightarrow \mathcal{L}(X, Y)$ an der Stelle $\varphi_{0}$ Fréchet-differenzierbar ist. Mit anderen Worten: wenn ein linearer und beschränkter Operator $F^{\prime \prime}\left(\varphi_{0}\right): X \rightarrow \mathcal{L}(X, Y)$ existiert, mit der Eigenschaft

$$
\left\|F^{\prime}\left(\varphi_{0}+h\right)-F^{\prime}\left(\varphi_{0}\right)-F^{\prime \prime}\left(\varphi_{0}, h\right)\right\|_{\mathcal{L}(X, Y)}=o\left(\|h\|_{X}\right), h \rightarrow 0 .
$$

Gleichung (2.7) lässt sich in ausführlicher Form schreiben als

$$
\sup _{\|\tilde{h}\|_{X}=1}\left\|F^{\prime}\left(\varphi_{0}+h, \tilde{h}\right)-F^{\prime}\left(\varphi_{0}, \tilde{h}\right)-F^{\prime \prime}\left(\varphi_{0}, h, \tilde{h}\right)\right\|_{Y}=o\left(\|h\|_{X}\right), h \rightarrow 0 .
$$

Da die Räume $\mathcal{L}(X, \mathcal{L}(X, Y))$ und $\mathcal{L}(X \times X, Y)$ isometrisch isomorph sind, lässt sich $F^{\prime \prime}\left(\varphi_{0}\right)$ als beschränkter bilinearer Operator von $X \times X$ nach $Y$ interpretieren.

Später werden lediglich Terme $F^{\prime \prime}\left(\varphi_{0}, h_{1}, h_{2}\right) \in Y$ mit $h_{1}=h_{2} \in X$ benötigt. In diesem Fall ist wegen der Isometrie eine abkürzende Schreibweise

$$
F^{\prime \prime}\left(\varphi_{0}, h\right):=F^{\prime \prime}\left(\varphi_{0}, h, h\right)
$$

naheliegend.

\subsection{Funktionalanalytische Grundlagen}

Satz 2.7 Sei $G \subset \mathbb{R}^{m}$ nichtleer, offen und beschränkt. Desweiteren sei

$$
k: \bar{G} \times \bar{G} \rightarrow \mathbb{C},(x, y) \mapsto k(x, y),
$$


stetig oder schwach singulär. Dann ist der Operator $A: C(\bar{G}) \rightarrow C(\bar{G})$, definiert durch

$$
(A \varphi)(x)=\int_{G} k(x, y) \varphi(y) d y, x \in \bar{G},
$$

linear, beschränkt und kompakt.

Beweis: siehe [3.5, Chapter 2]. 


\section{Kapitel 3}

\section{Eine singuläre Integralgleichung}

Die Behandlung von Randwertproblemen für $C^{2}$-glatte Gebiete $D$ im $\mathbb{R}^{2}$ mittels Integralgleichungsmethoden führt nach einer Parametrisierung auf Integralgleichungen mit stetigen oder schwach singulären Kernen. Weist das Gebiet $D$ hingegen Ecken auf, so spiegelt sich das im singulären Verhalten der Kerne wider. Für eine Behandlung der Randintegralgleichung (4.12) im Kapitel 4 ist es daher erforderlich, auf diese Problematik einzugehen. An dieser Stelle ist nicht die Kenntnis über die exakte Form der Gleichung (4.12) erforderlich. Es soll vielmehr, losgelöst vom konkreten Problem, ausschließlich mit funktionalanalytischen Hilfsmitteln gearbeitet werden.

Daher wird in diesem Kapitel eine singuläre Integralgleichung zweiter Art behandelt, deren Kern sich in den Ecken des Quadrats $[0,2 \pi] \times[0,2 \pi]$ wie ein Mellin-Kern verhält. Das singuläre Verhalten des Kerns spiegelt sich in den Singularitäten der Ableitungen der Lösung wider.

\subsection{Zur Existenz und Eindeutigkeit}

Betrachte die Gleichung zweiter Art

$$
\begin{aligned}
\varphi(t)- & \int_{0}^{2 \pi}\left\{K_{A}(t, \tau)+K_{B}(t, \tau)+K_{C}(t, \tau) \ln \left(4 \sin ^{2} \frac{t-\tau}{2}\right)\right\}[\varphi(\tau)-\varphi(0)] d \tau \\
& +\gamma(t) \varphi(0)=f(t), 0 \leq t \leq 2 \pi
\end{aligned}
$$

bei gegebenen $f, \gamma \in C_{2 \pi}(\mathbb{R}, \mathbb{R})$ und den in beiden Variablen $2 \pi$-periodischen Kernen $K_{A}, K_{B}, K_{C}$ 
An die Kerne werden die folgenden Voraussetzungen gestellt:

1. $K_{C} \in C_{2 \pi}(\mathbb{R} \times \mathbb{R}, \mathbb{R})$,

2. $K_{B}$ ist beschränkt auf $[0,2 \pi] \times[0,2 \pi]$ und dort mit Ausnahme der vier Ecken stetig,

3. $K_{A}$ ist stetig auf $(0,2 \pi] \times(0,2 \pi]$,

4. $K_{A} \geq 0$ oder $K_{A} \leq 0$,

5. $K_{A}$ hat Träger in $[0, \delta] \times[0, \delta], 0<\delta<2 \pi$.

Desweiteren existiere eine beschränkte, stetig differenzierbare Funktion

$$
k:[0, \infty) \rightarrow[0, \infty)
$$

mit beschränkter Ableitung $k^{\prime}$ und

$$
\begin{aligned}
k(0) & =0, \\
k(s) & =O\left(\frac{1}{s}\right), s \rightarrow \infty, \\
\left|K_{A}(t, \tau)\right| & \leq \frac{1}{\tau} k\left(\frac{t}{\tau}\right) \text { für } \tau>0, \\
\int_{0}^{\infty} \frac{k(s)}{s} d s & <1 .
\end{aligned}
$$

Mit den Integraloperatoren

$$
\begin{aligned}
(A \varphi)(t) & :=\int_{0}^{2 \pi} K_{A}(t, \tau)[\varphi(\tau)-\varphi(0)] d \tau \\
(B \varphi)(t) & :=\int_{0}^{2 \pi} K_{B}(t, \tau)[\varphi(\tau)-\varphi(0)] d \tau-\gamma(t) \varphi(0), \\
(C \varphi)(t) & :=\int_{0}^{2 \pi} K_{C}(t, \tau) \ln \left(\sin ^{2} \frac{t-\tau}{2}\right)[\varphi(\tau)-\varphi(0)] d \tau,
\end{aligned}
$$

erhält die Integralgleichung (3.1) die Form

$$
(I-A-B-C) \varphi=f .
$$


Mit der zur gewöhnlichen Supremumnorm äquivalenten Norm

$$
\|\varphi\|_{\infty, 0}:=\max _{0 \leq t \leq 2 \pi}|\varphi(t)-\varphi(0)|+|\varphi(0)|
$$

wird $C_{2 \pi}:=C_{2 \pi}(\mathbb{R}, \mathbb{R})$ ein Banachraum.

Satz 3.1 Die durch (3.4) - (3.6) erklärten Operatoren A, B, C haben die Abbildungseigenschaft

$$
A, B, C: C_{2 \pi} \rightarrow C_{2 \pi},
$$

sind linear und beschränkt. Weiterhin sind $B, C$ kompakt und es gilt

$$
\|A\|_{\infty, 0} \leq \int_{0}^{\infty} \frac{k(s)}{s} d s<1 .
$$

Beweis: Die Resultate für $B$ und $C$ ergeben sich aus den Sätzen über schwach singuläre Kerne. Die Eigenschaften des Operators $A$ sind in [28] oder [55] nachgewiesen worden.

Der Satz über die Neumannsche Reihe zusammen mit Satz 3.1 liefern das nachfolgende Korollar.

Korollar 3.2 Für die Operatorgleichung $(I-A-B-C) \varphi=f$ lässt sich die Riesz-Theorie anwenden.

\subsection{Die Substitutionsmethode}

Eine Konsequenz der Euler - Maclaurin Formel ist die, dass die Trapezregel zur numerischen Integration von periodischen und hinreichend oft stetig differenzierbaren Funktionen gut geeignet ist. Für Integranden mit (integrierbaren) Endpunktsingularitäten bleibt dieses Resultat nicht richtig. Gesucht wird

$$
\int_{0}^{2 \pi} g(t) d t
$$

wobei $g:(0,2 \pi) \rightarrow \mathbb{R}$ hinreichend glatt ist, aber in den Endpunkten $t=0$ und $t=2 \pi$ Singularitäten besitzt. Sei

$$
w:[0,2 \pi] \rightarrow[0,2 \pi]
$$


bijektiv, streng monoton wachsend und hinreichend oft differenzierbar. Desweiteren haben $w$ in 0 und $2 \pi-w$ in $2 \pi$ Nullstellen höherer Ordnung. Aufgrund der Substitution in

$$
\int_{0}^{2 \pi} g(t) d t=\int_{0}^{2 \pi} w^{\prime}(s) g(w(s)) d s
$$

liefert die Trapezregel, angewendet auf den substituierten Integranden, eine Quadraturformel

$$
\int_{0}^{2 \pi} g(t) d t \approx \frac{\pi}{n} \sum_{j=1}^{2 n-1} \alpha_{j}^{(n)} g\left(s_{j}^{(n)}\right)=: Q_{n}(g)
$$

mit den Gewichten

$$
\alpha_{j}^{(n)}=w^{\prime}\left(\frac{j \pi}{n}\right), j=1, \ldots, 2 n-1
$$

und den Stützstellen

$$
s_{j}^{(n)}=w\left(\frac{j \pi}{n}\right), j=1, \ldots, 2 n-1 .
$$

Aufgrund der Wahl von $w$ verschwindet der substituierte Integrand an den Intervallenden, sodass die Randterme in (3.8) nicht auftreten.

Die angegebenen Forderungen an die Substitution sollen nun konkretisiert werden.

Definition 3.3 Es sei $w:[0,2 \pi] \rightarrow[0,2 \pi]$ eine bijektive, streng monoton wachsende Funktion. Dann heißt $w$ zulässig mit Parameter $p \in \mathbb{N}$, falls $w \in C^{p+1}[0,2 \pi]$,

$$
\begin{aligned}
2 \pi-w(t) & =w(t), 0 \leq t \leq 2 \pi, \\
w^{(j)}(0)=w^{(j)}(2 \pi) & =0 \text { für } j=1, \ldots, p-1
\end{aligned}
$$

und $w^{(p)}(0) \neq 0, w^{(p)}(2 \pi) \neq 0$ ist.

In Vogt [55] wurden einige Substitutionen untersucht. Für die numerischen Ergebnisse in der vorliegenden Arbeit wurden die von Korobov vorgeschlagene polynomiale Substitution

$$
w_{p}(t)=\frac{2 p-1}{(2 \pi)^{2 p-2}}\left(\begin{array}{c}
2 p-2 \\
p-1
\end{array}\right) \int_{0}^{t}[u(2 \pi-u)]^{p-1} d u, 0 \leq t \leq 2 \pi,
$$

die von Sidi [53] vorgeschlagene trigonometrische Substitution

$$
w_{p}(t)=\frac{2 \pi}{\vartheta_{p}(2 \pi)} \vartheta_{p}(t), \vartheta_{p}(t)=\int_{0}^{t}\left(\sin \frac{u}{2}\right)^{p-1} d u
$$


sowie die von Kreß vorgeschlagene rationale Substitution

$$
w_{p}(t)=2 \pi \frac{[v(t)]^{p}}{[v(t)]^{p}+[v(2 \pi-t)]^{p}}, 0 \leq t \leq 2 \pi, p \geq 2,
$$

mit dem kubischen Polynom

$$
v(t)=\left(\frac{2}{p}-1\right) \frac{(\pi-t)^{3}}{\pi^{2}}+\frac{2}{p}(t-\pi)+\pi, 0 \leq t \leq 2 \pi, p \geq 2,
$$

verwendet.

Der passende Funktionenraum für die Anwendung der Quadraturformel (3.8) ist

$$
S^{q, \alpha}=\left\{g \in C^{q}(0,2 \pi)\left|\int_{0}^{2 \pi}[t(2 \pi-t)]^{j-\alpha}\right| g^{(j)}(t) \mid d t \text { existiert für } j=0, \ldots, q\right\},
$$

wobei für die Parameter gelte $q \in \mathbb{N}$ und $0<\alpha \leq q . S^{q, \alpha}$ wird mit der Norm

$$
\|g\|_{q, \alpha}:=\max _{j=0, \ldots, q} \int_{0}^{2 \pi}[t(2 \pi-t)]^{j-\alpha}\left|g^{(j)}(t)\right| d t
$$

ein normierter Raum.

Der nachfolgende Satz macht eine Aussage über den Quadraturfehler für Funktionen aus $S^{q, \alpha}$.

Satz 3.4 Seien $g \in S^{2 q+1, \alpha}$ mit $0<\alpha \leq q$ und $w$ zulässig mit Parameter $p \in \mathbb{N}$ derart, dass

$$
\alpha p \geq 2 q+1 \text { und } p>2 q+1
$$

Dann gilt für den Fehler

$$
E_{n, p}(g):=\frac{\pi}{n} \sum_{j=1}^{2 n-1} \alpha_{j}^{(n)} g\left(s_{j}^{(n)}\right)-\int_{0}^{2 \pi} g(t) d t
$$

in der Quadraturformel (3.8)

$$
\left|E_{n, p}(g)\right| \leq \frac{C}{n^{2 q+1}}\|g\|_{2 q+1, \alpha}
$$

mit einer Konstanten $C=C(w, \alpha, q)$. 
Beweis: Für den Beweis sei auf [28] oder [55] verwiesen.

Bemerkung 3.5 In [94] wird gezeigt, dass sich bei modifizierter Definition des Raumes $S^{q, \alpha}$ und der speziellen Substitution $w$, mit

$$
w(z)=\frac{1}{1+e^{-z}},
$$

die die Differenzialgleichung $w^{\prime}=w(1-w)$ erfüllt, für die Quadraturformel

$$
\int_{0}^{1} g(t) d t \approx h \sum_{j=-n}^{n} w^{\prime}(j h) g(w(j h))=: Q_{n, h}(g)
$$

eine exponentielle Fehlerabschätzung gewinnen lässt.

Für den Integraloperator $C$ gemäß (3.6) ist eine numerische Auswertung mit einer Trapezregel beziehungsweise „gewichteten“ Trapezregel (3.8) nicht vielversprechend. Zur Approximation von Integralen mit schwach singulärer Gewichtsfunktion, hier $\ln \left(4 \sin ^{2} \frac{t-\tau}{2}\right)$, werden Quadraturformeln benötigt, deren Gewichte wiederum Funktionen sind. Eine ausführliche Darstellung findet man bei Sloan [54]. Für die hier vorliegende logarithmische Singularität wird wie in Kussmaul [38] und Martensen [42] vorgegangen.

Sei dazu $g \in C_{2 \pi}$. Die Quadraturformel

$$
\begin{aligned}
(Q g)(t) & =\int_{0}^{2 \pi} \ln \left(4 \sin ^{2} \frac{t-\tau}{2}\right) g(\tau) d \tau \\
& \approx \int_{0}^{2 \pi} \ln \left(4 \sin ^{2} \frac{t-\tau}{2}\right)\left(P_{n} g\right)(\tau) d \tau=:\left(Q_{n} g\right)(t),
\end{aligned}
$$

mit dem trigonometrischen Interpolationsoperator

$$
\begin{aligned}
& P_{n}: \quad C_{2 \pi} \rightarrow T_{n}:=\left\{\frac{\alpha_{0}}{2}+\sum_{k=1}^{n-1}\left[\alpha_{k} \cos k t+\beta_{k} \sin k t\right]+\frac{\alpha_{n}}{2} \cos n t \mid \alpha_{k}, \beta_{k} \in \mathbb{R}\right\}, \\
& P_{n} g=\sum_{j=0}^{2 n-1} g\left(t_{j}^{(n)}\right) l_{j}^{(n)}, \quad l_{j}^{(n)}\left(t_{k}^{(n)}\right)=\delta_{j k},
\end{aligned}
$$

zu den äquidistanten Stützstellen $t_{j}^{(n)}=j \pi / n, j=0, \ldots, 2 n-1$, führt auf

$$
\left(Q_{n} g\right)(t)=\sum_{j=0}^{2 n-1} R_{j}^{(n)}(t) g\left(t_{j}^{(n)}\right)
$$


mit den Gewichten

$$
\begin{aligned}
R_{j}^{(n)}(t) & =\int_{0}^{2 \pi} \ln \left(4 \sin ^{2} \frac{t-\tau}{2}\right) l_{j}^{(n)}(\tau) d \tau \\
& =-\frac{2 \pi}{n} \sum_{m=1}^{n-1} \frac{1}{m} \cos m\left(t-t_{j}^{(n)}\right)-\frac{\pi}{n^{2}} \cos n\left(t-t_{j}^{(n)}\right), j=0, \ldots, 2 n-1,
\end{aligned}
$$

wobei die Lagrangebasis $l_{j}^{(n)}$ gegeben ist durch

$$
\begin{aligned}
l_{j}^{(n)}(t) & =\operatorname{Re} \frac{1}{2 n}\left\{1+2 \sum_{k=1}^{n-1} e^{i k\left(t-t_{j}^{(n)}\right)}+e^{i n\left(t-t_{j}^{(n)}\right)}\right\} \\
& =\operatorname{Re} \frac{1}{2 n} i\left(1-e^{i n\left(t-t_{j}^{(n)}\right)}\right) \cot \frac{t-t_{j}^{(n)}}{2} \\
& =\frac{1}{2 n} \sin n\left(t-t_{j}^{(n)}\right) \cot \frac{t-t_{j}^{(n)}}{2}, t \neq t_{j}^{(n)}, j=0, \ldots, 2 n-1 .
\end{aligned}
$$

Um die Quadraturformeln (3.8) und (3.15) gleichzeitig auf die Gleichung (3.7) anwenden zu können, ist auch beim logarithmischen Anteil eine Substitution erforderlich.

Mit $t=w(s)$ und $\tau=w(\sigma)$ gilt

$$
\begin{aligned}
& \int_{0}^{2 \pi} \ln \left(4 \sin ^{2} \frac{t-\tau}{2}\right) g(\tau) d \tau \\
& =\int_{0}^{2 \pi} \ln \left(4 \sin ^{2} \frac{w(s)-w(\sigma)}{2}\right) g(w(\sigma)) w^{\prime}(\sigma) d \sigma \\
& =\int_{0}^{2 \pi} \ln \left(4 \sin ^{2} \frac{s-\sigma}{2}\right) g(w(\sigma)) w^{\prime}(\sigma) d \sigma+\int_{0}^{2 \pi} T(s, \sigma) g(w(\sigma)) w^{\prime}(\sigma) d \sigma \\
& \approx \sum_{j=1}^{2 n-1} R_{j}^{(n)}(s) \alpha_{j}^{(n)} g\left(s_{j}^{(n)}\right)+\frac{\pi}{n} \sum_{j=1}^{2 n-1} T\left(s, t_{j}^{(n)}\right) \alpha_{j}^{(n)} g\left(s_{j}^{(n)}\right)
\end{aligned}
$$

mit der stetigen Funktion

$$
w^{\prime} T:[0,2 \pi] \times[0,2 \pi] \rightarrow \mathbb{R},(s, \sigma) \mapsto w^{\prime}(\sigma) T(s, \sigma),
$$


wobei $T$ erklärt ist durch

$$
T(s, \sigma)=\left\{\begin{array}{cl}
\ln \left(\frac{\left.\sin ^{2} \frac{w(s)-w(\sigma)}{2}\right)}{\sin ^{2} \frac{s-\sigma}{2}}\right): & 0 \leq s, \sigma \leq 2 \pi,|s-\sigma| \notin\{0,2 \pi\}, \\
2 \ln w^{\prime}(s) & : 0<s=\sigma<2 \pi .
\end{array}\right.
$$

\subsection{Ein Nyström - Verfahren}

Eine Möglichkeit (3.7) approximativ zu lösen besteht darin, die Integrale zu ersetzen durch die Quadraturformeln (3.8) und (3.17). Für den Rest des Kapitels werden von $w_{p}$ die Eigenschaften aus Definition 3.3 gefordert.

Für $n \in \mathbb{N}$ ist $\varphi_{n} \in C_{2 \pi}$ so zu bestimmen, dass für $0 \leq t \leq 2 \pi$ gilt

$$
\begin{aligned}
\varphi_{n}(t)- & \frac{\pi}{n} \sum_{j=1}^{2 n-1}\left\{\left(K_{A}+K_{B}\right)\left(t, s_{j}^{(n)}\right)+K_{C}\left(t, s_{j}^{(n)}\right)\left[T\left(w^{-1}(t), t_{j}^{(n)}\right)+\frac{n}{\pi} R_{j}^{(n)}\left(w^{-1}(t)\right)\right]\right\} \times \\
& \times \alpha_{j}^{(n)}\left[\varphi\left(s_{j}^{(n)}\right)-\varphi(0)\right]+\gamma(t) \varphi(0)=f(t),
\end{aligned}
$$

wobei weiterhin die Bezeichnungen $t_{j}^{(n)}=j \pi / n, s_{j}^{(n)}=w\left(t_{j}^{(n)}\right)$ und $\alpha_{j}^{(n)}=w^{\prime}\left(t_{j}^{(n)}\right)$ gelten. Für jede Lösung $\varphi_{n} \in C_{2 \pi}$ von (3.19) erfüllen die Werte $\varphi_{l}^{(n)}:=\varphi\left(s_{l}^{(n)}\right), l=0, \ldots, 2 n-1$, das lineare Gleichungssystem

$$
\begin{aligned}
\varphi_{l}^{(n)}- & \frac{\pi}{n} \sum_{j=1}^{2 n-1}\left\{\left(K_{A}+K_{B}\right)\left(s_{l}^{(n)}, s_{j}^{(n)}\right)+K_{C}\left(s_{l}^{(n)}, s_{j}^{(n)}\right)\left[T\left(t_{l}^{(n)}, t_{j}^{(n)}\right)+\frac{n}{\pi} R_{|j-l|}^{(n)}\right]\right\} \times \\
& \times \alpha_{j}^{(n)}\left[\varphi_{j}^{(n)}-\varphi_{0}^{(n)}\right]+\gamma\left(s_{l}^{(n)}\right) \varphi_{0}^{(n)}=f\left(s_{l}^{(n)}\right),
\end{aligned}
$$

wobei für die Matrixelemente $R_{|j-l|}^{(n)}:=R_{j}^{(n)}\left(t_{l}^{(n)}\right)$ gilt

$$
R_{k}^{(n)}=-\frac{2 \pi}{n} \sum_{m=1}^{n-1} \frac{1}{m} \cos \left(\frac{m k \pi}{n}\right)-\frac{(-1)^{k} \pi}{n^{2}}, k=0, \ldots, 2 n-1 .
$$

Umgekehrt erhält man für eine Lösung $\varphi_{0}^{(n)}, \ldots, \varphi_{2 n-1}^{(n)}$ des Gleichungssystems (3.20) durch

$$
\begin{aligned}
\varphi_{n}(t):= & \frac{\pi}{n} \sum_{j=1}^{2 n-1}\left\{\left(K_{A}+K_{B}\right)\left(t, s_{j}^{(n)}\right)+K_{C}\left(t, s_{j}^{(n)}\right)\left[T\left(w^{-1}(t), t_{j}^{(n)}\right)+\frac{n}{\pi} R_{j}^{(n)}\left(w^{-1}(t)\right)\right]\right\} \times \\
& \times \alpha_{j}^{(n)}\left[\varphi_{j}^{(n)}-\varphi_{0}^{(n)}\right]+f(t)-\gamma(t) \varphi_{0}^{(n)}
\end{aligned}
$$


eine Lösung von (3.19), denn $\varphi_{n}\left(s_{i}^{(n)}\right)=\varphi_{i}^{(n)}$.

Um eine Konvergenzaussage formulieren zu können, werden weitere Voraussetzungen an die Kerne gestellt. Dazu gelte

- $K_{B}$ und $K_{C}$ sind bezüglich der zweiten Variablen $2 q+1$-mal stetig differenzierbar auf $[0,2 \pi] \times[0,2 \pi]$.

- Für den im Punkt $(0,0)$ singulären Kern $K_{A}$ existieren die partiellen Ableitungen bezüglich $\tau$ bis zur Ordnung $2 q+1$ und diese sind stetig auf $[0,2 \pi] \times[0,2 \pi] \backslash\{(0,0)\}$.

- Es existieren Funktionen

$$
k_{l}:[0, \infty) \rightarrow[0, \infty), l=0, \ldots, 2 q+1,
$$

mit den Eigenschaften

$$
\begin{aligned}
k_{l}(s) & =O\left(\frac{1}{s}\right), s \rightarrow \infty \\
\left|\frac{\partial^{l}}{\partial \tau^{l}} K_{A}(t, \tau)\right| & \leq \frac{1}{\tau^{l+1}} k_{l}\left(\frac{t}{\tau}\right), \tau>0 .
\end{aligned}
$$

Mit den Operatoren

$$
\begin{aligned}
\left(A_{n} \varphi\right)(t) & =\frac{\pi}{n} \sum_{j=1}^{2 n-1} \alpha_{j}^{(n)} K_{A}\left(t, s_{j}^{(n)}\right)\left[\varphi\left(s_{j}^{(n)}\right)-\varphi(0)\right], \\
\left(B_{n} \varphi\right)(t) & =\frac{\pi}{n} \sum_{j=1}^{2 n-1} \alpha_{j}^{(n)} K_{B}\left(t, s_{j}^{(n)}\right)\left[\varphi\left(s_{j}^{(n)}\right)-\varphi(0)\right]-\gamma(t) \varphi(0), \\
\left(C_{n} \varphi\right)(t) & =\frac{\pi}{n} \sum_{j=1}^{2 n-1} \alpha_{j}^{(n)} K_{C}\left(t, s_{j}^{(n)}\right)\left\{T\left(w^{-1}(t), t_{j}^{(n)}\right)+\frac{n}{\pi} R_{j}^{(n)}\left(w^{-1}(t)\right)\right\}\left[\varphi\left(s_{j}^{(n)}\right)-\varphi(0)\right]
\end{aligned}
$$

wird im nachfolgenden Satz ein Konvergenzresultat zitiert. Vorab werden mit einer stetigen Funktion $g:[0, \infty) \rightarrow[0,1]$, mit

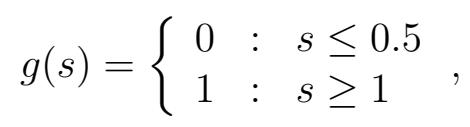


die Operatoren $A_{n}$ modifiziert, das heißt für $r \in\{1,2, \ldots, 2 n-1\}$ werden die Operatoren $A_{n, r}: C_{2 \pi} \rightarrow C_{2 \pi}$ erklärt durch

$$
\left(A_{n, r} \varphi\right)(t)=g\left(\frac{t}{s_{r}^{(n)}}\right)\left(A_{n} \varphi\right)(t), 0 \leq t \leq 2 \pi .
$$

Satz 3.6 Seien $0<\alpha<1, \alpha p \geq 2 q+1$. Für die Lösung der Gleichung

$$
(I-A-B-C) \varphi=f
$$

gelte $\varphi-\varphi(0) \in S^{2 q+1, \alpha+1}$. Dann existiert ein $r \in \mathbb{N}$ so, dass für alle $n \geq r$ die Operatoren $I-A_{n, r}-B_{n}-C_{n}$ beschränkt invertierbar sind. Insbesondere besitzt

$$
\left(I-A_{n, r}-B_{n}-C_{n}\right) \varphi_{n}=f
$$

genau eine Lösung $\varphi_{n} \in C_{2 \pi}$. Es gilt die Fehlerabschätzung

$$
\left\|\varphi_{n}-\varphi\right\|_{\infty} \leq C(\alpha, p, q) \frac{\ln (n)}{n^{2 q-1}}\|\varphi-\varphi(0)\|_{2 q+1, \alpha+1}
$$

mit einer von $n$ und $\varphi$ unabhängigen Konstanten $C(\alpha, p, q)$.

Beweis: Der Beweis soll nur skizziert werden. Für eine ausführliche Darstellung sei auf Kümpel [39] verwiesen. Wie in Kreß [28] erhält man zunächst mit dem Integral (3.3) und dem Satz über die Neumannsche Reihe ein $r>0$ so, dass die Operatoren $\left(I-A_{n, r}\right)^{-1}$ für $n \geq r$ existieren und gleichmäßig beschränkt sind. Mit den zusätzlich vorausgesetzten Glätteeigenschaften der Kerne $K_{A}, K_{B}, K_{C}$ lässt sich punktweise Konvergenz der Operatoren $\left(A_{n}\right)_{n \in \mathbb{N}},\left(B_{n}\right)_{n \in \mathbb{N}},\left(C_{n}\right)_{n \in \mathbb{N}}$ folgern, das heißt

$$
\begin{aligned}
\left\|\left(A-A_{n}\right) \psi\right\|_{\infty, 0} & \leq \frac{c}{n^{2 q+1}}\|\psi-\psi(0)\|_{2 q+1, \alpha+1}, \\
\left\|\left(B-B_{n}\right) \psi\right\|_{\infty, 0} & \leq \frac{c}{n^{2 q+1}}\|\psi-\psi(0)\|_{2 q+1, \alpha+1}, \\
\left\|\left(C-C_{n}\right) \psi\right\|_{\infty, 0} & \leq \frac{c \ln n}{n^{2 q-1}}\|\psi-\psi(0)\|_{2 q+1, \alpha+1},
\end{aligned}
$$

vorausgesetzt $\psi-\psi(0) \in S^{2 q+1, \alpha+1}$. Mit der Kollektiv-Kompaktheit der Folgen $\left(B_{n}\right)_{n \in \mathbb{N}}$, $\left(C_{n}\right)_{n \in \mathbb{N}}$ und der Dreiecksungleichung

$$
\left\|\left(A-A_{n, r}\right) \psi\right\|_{\infty, 0} \leq\left\|\left(A-A_{n}\right) \psi\right\|_{\infty, 0}+\sup _{0 \leq t \leq s_{r}^{(n)}}|(A \psi)(t)|
$$

ergibt sich die Behauptung aus Kreß [35, Theorem 10.8 und Problem 10.3]. 


\section{Kapitel 4}

\section{Das direkte Streuproblem}

Seien $D \subset \mathbb{R}^{2}$ ein beschränktes Gebiet, $\mathbb{R}^{2} \backslash D$ zusammenhängend und $x_{0}, x_{1}, \ldots, x_{m}$ endlich viele Punkte aus $\partial D$ so, dass gilt $\partial D \backslash\left\{x_{0}, x_{1}, \ldots, x_{m}\right\}$ besteht aus $m+1$ Randstücken der Klasse $C^{2}$.

Mit $\Gamma_{j}:=\overline{x_{j}, x_{j+1}}, j=0, \ldots, m-1, \Gamma_{m}:=\overline{x_{m}, x_{0}}$ werden die $C^{2}$-glatten Randstücke bezeichnet. Für Punkte $x \in \partial D$ werden mit $\beta(x)$ die Innenwinkel bei $x$ bezeichnet. Insbesondere gelte $0<\beta\left(x_{j}\right)<2 \pi, j=0, \ldots, m$ und $\beta(x)=\pi, x \in \partial D \backslash\left\{x_{0}, x_{1}, \ldots, x_{m}\right\}$.

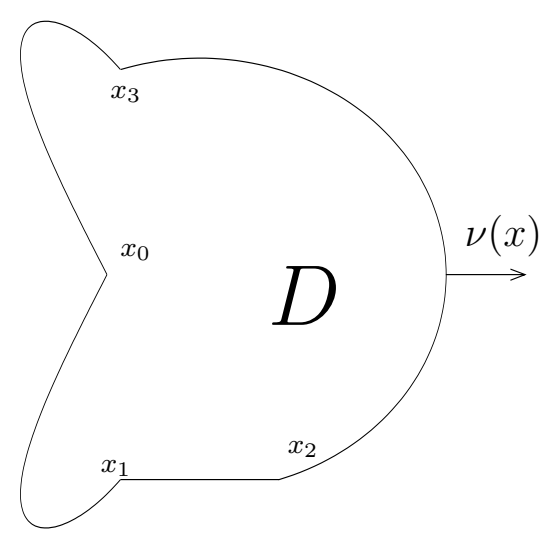

Für die Wellenzahl $k \in \mathbb{C} \backslash\{0\}$ setzen wir $k \in \mathbb{R}^{+}$oder $\operatorname{Im}(k)>0, \operatorname{Re}(k) \geq 0$ voraus. Die Normale $\nu(x)$ (in den regulären Randpunkten) weise in das unbeschränkte Außengebiet. 


\subsection{Randwertprobleme zur Helmholtzgleichung}

\section{Definition 4.1 (Äußeres Dirichletproblem)}

Gegeben ist $f \in C(\partial D)$. Gesucht wird eine Funktion $u \in C^{2}\left(\mathbb{R}^{2} \backslash \bar{D}\right) \cap C\left(\mathbb{R}^{2} \backslash D\right)$ als Lösung der Helmholtzgleichung

$$
\triangle u+k^{2} u=0 \quad \text { in } \mathbb{R}^{2} \backslash \bar{D},
$$

die die Randbedingung

$$
u=f \quad \text { auf } \partial D
$$

und die Sommerfeldsche Ausstrahlungsbedingung (SAB)

$$
\lim _{r \rightarrow \infty} r^{1 / 2}\left(\frac{\partial u}{\partial r}-i k u\right)=0, r=|x|
$$

gleichmäßig für alle Richtungen $\hat{x}=\frac{x}{|x|}$ erfüllt.

\section{Definition 4.2 (Inneres Neumannproblem)}

Gegeben ist $g \in C(\partial D)$. Gesucht wird eine Funktion $u \in C^{2}(D) \cap C(\bar{D})$, deren Normalableitung

$$
\frac{\partial u_{-}(x)}{\partial \nu}:=\lim _{h \downarrow 0} \operatorname{grad} u(x-h \nu(x)) \nu(x), x \in \partial D \backslash\left\{x_{0}, \ldots, x_{m}\right\}
$$

in den regulären Randpunkten existiert, die in D die Helmholtzgleichung löst und die Randbedingung

$$
\frac{\partial u_{-}(x)}{\partial \nu}=g(x), x \in \partial D \backslash\left\{x_{0}, \ldots, x_{m}\right\}
$$

erfüllt.

\subsection{Zur Eindeutigkeit beim äußeren Dirichlet-Problem}

Prinzipiell lässt sich der Eindeutigkeitsbeweis wie in [7, Lemma 3.8] führen, da der Satz von Gauß auf Funktionen aus $C_{0}^{1}\left(D_{R}\right)$ angewendet wird. An dieser Stelle soll jedoch ein anderer Weg beschritten werden, der auch auf andere Differenzialgleichungen anwendbar ist. 
Lemma 4.3 Für $\varepsilon_{1}>0$ sei $\left.\left.\tilde{f}:\right] 0, \varepsilon_{1}\right] \rightarrow \mathbb{R}$ monoton fallend, differenzierbar und erfülle die Differenzialungleichung

$$
|\tilde{f}(\varepsilon)| \leq C_{1} \varepsilon^{2}+C_{2} \sqrt{-\varepsilon \tilde{f}^{\prime}(\varepsilon)}, 0<\varepsilon \leq \varepsilon_{1}
$$

mit von $\varepsilon$ unabhängigen Konstanten $C_{1} \geq 0, C_{2}>0$. Dann existiert der rechtsseitige Grenzwert

$$
\tilde{f}(0):=\lim _{\varepsilon \rightarrow 0+} \tilde{f}(\varepsilon)
$$

und ist Null.

Beweis: Siehe [24] oder im Fall $C_{1}=0$ [5.5, Lemma 5.2].

Für den Eindeutigkeitsbeweis führen wir die Bezeichnungen $D_{\varepsilon, R}$ und $\Gamma_{\varepsilon}$ ein. Dazu sei $R>0$ so groß, dass $\bar{D}$ in der offenen Kreisscheibe $B(0, R)$ enthalten ist.

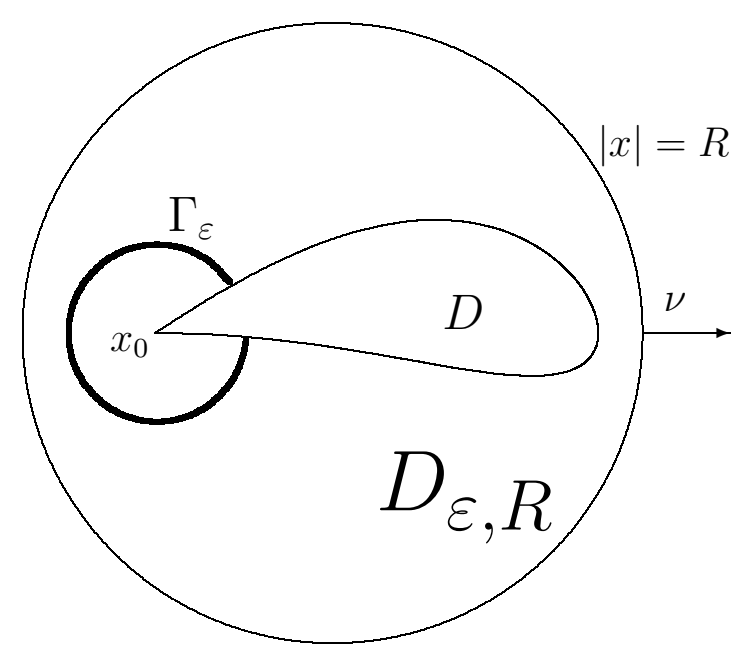

Abbildung: Darstellung für den Fall genau einer Ecke.

Für hinreichend kleines $\varepsilon>0$ ist die Menge

$$
D_{\varepsilon, R}:=\left\{x \in \mathbb{R}^{2} \backslash \bar{D}|| x|<R,| x-x_{j} \mid>\varepsilon \text { für alle } j=0, \ldots, m\right\}
$$

ebenfalls ein Gebiet und

$$
\Gamma_{\varepsilon}:=\left\{x \in \mathbb{R}^{2} \backslash \bar{D} \mid \exists j \in\{0, \ldots, m\} \text { mit }\left|x-x_{j}\right|=\varepsilon\right\}
$$


besteht aus $m+1$ glatten Kurvenstücken. Für die Normalenrichtung auf dem Kurvenstück $\Gamma_{\varepsilon}$ wird nachfolgendes vereinbart: Bei Oberflächenintegralen, bei dem der Integrationsbereich der Rand eines Gebiets ist, weist die Normale in das Außengebiet. Tritt das Integral über $\Gamma_{\varepsilon}$ separat auf, so wird auf dem Teil von $\Gamma_{\varepsilon}$ mit $\left|x-x_{j}\right|=\varepsilon$ die Normale durch $\nu(x)=\frac{x-x_{j}}{\varepsilon}$ gegeben.

Lemma 4.4 Für $\varepsilon_{1}>0$ sei $u \in C\left(\overline{D_{\varepsilon_{1}, R}}\right)$. Dann ist

$$
\tilde{f}(\varepsilon):=\int_{D_{\varepsilon, R}} u(x) d x, \varepsilon>\varepsilon_{1}
$$

differenzierbar mit Ableitung

$$
\tilde{f}^{\prime}(\varepsilon)=-\int_{\Gamma_{\varepsilon}} u(x) d s(x)
$$

Beweis: Siehe [5.5, Lemma 5.1].

Satz 4.5 Das äußere Dirichletproblem (4.1) - (4.3) besitzt höchstens eine Lösung.

Beweis: Aufgrund der Linearität genügt der Nachweis der Eindeutigkeit für das homogene Problem. Sei $u$ Lösung zu $f=0$. Für hinreichend kleines $\varepsilon>0$ und großes $R>0$ ist $D_{\varepsilon, R}$ ein Gebiet mit stückweise glattem Rand. Es ist bekannt (siehe Bemerkung 4.7), dass $u$ bis in den Rand von $D_{\varepsilon, R}$ differenzierbar ist. Der erste Greensche Satz angewendet auf das Gebiet $D_{\varepsilon, R}$ und die Funktionen $u, \bar{u}$ liefert

$$
\int_{D_{\varepsilon, R}}\left[|\operatorname{grad} u|^{2}-\bar{k}^{2}|u|^{2}\right] d x=\int_{\partial D_{\varepsilon, R}} u \frac{\partial \bar{u}}{\partial \nu} d s
$$

Wir bilden den Realteil dieser Gleichung und erhalten für

$$
\tilde{f}(\varepsilon):=\int_{D_{\varepsilon, R}}|\operatorname{grad} u|^{2} d x-\operatorname{Re}\left(\bar{k}^{2}\right) \int_{|x| \leq R, x \notin D}|u|^{2} d x-\operatorname{Re} \int_{|x|=R} u \frac{\partial \bar{u}}{\partial \nu} d s
$$

die Beziehung

$$
\tilde{f}(\varepsilon)=-\operatorname{Re}\left(\bar{k}^{2}\right) \sum_{j=0}^{m} \int_{\left|x-x_{j}\right| \leq \varepsilon, x \notin D}|u|^{2} d x-\operatorname{Re} \int_{\Gamma_{\varepsilon}} u \frac{\partial \bar{u}}{\partial \nu} d s .
$$


Die auf $\left.] 0, \varepsilon_{1}\right]$ definierte Funktion $\tilde{f}$ ist monoton fallend (Das Gebiet $D_{\varepsilon, R}$ wird mit wachsendem $\varepsilon$ kleiner.) und ist nach Lemma 4.4 differenzierbar mit Ableitung

$$
\tilde{f}^{\prime}(\varepsilon)=-\int_{\Gamma_{\varepsilon}}|\operatorname{grad} u|^{2} d s
$$

Es verbleibt der Nachweis, dass $\tilde{f}$ eine Differenzialungleichung wie in Lemma 4.3 erfüllt. Mit $M=\|u\|_{\infty, B[0, R] \backslash D}$ liefert die Cauchy-Schwarzsche Ungleichung angewendet auf den zweiten Summanden in (4.7)

$$
\begin{aligned}
|\tilde{f}(\varepsilon)| & \leq|k|^{2}(m+1) \pi \varepsilon^{2} M^{2}+\left(\int_{\Gamma_{\varepsilon}}|u|^{2} d s\right)^{1 / 2}\left(\int_{\Gamma_{\varepsilon}}\left|\frac{\partial u}{\partial \nu}\right|^{2} d s\right)^{1 / 2} \\
& \leq C_{1} \varepsilon^{2}+M \sqrt{m+1} \sqrt{2 \pi \varepsilon} \sqrt{-\tilde{f}^{\prime}(\varepsilon)} \\
& =C_{1} \varepsilon^{2}+C_{2} \sqrt{-\varepsilon \tilde{f}^{\prime}(\varepsilon)}
\end{aligned}
$$

Demnach ist $\lim _{\varepsilon \rightarrow 0+} \tilde{f}(\varepsilon)=0$. Da der erste Summand in der Darstellung (4.7) quadratisch gegen Null strebt, folgt für den zweiten Summanden

$$
\lim _{\varepsilon \rightarrow 0} \operatorname{Re} \int_{\Gamma_{\varepsilon}} u \frac{\partial \bar{u}}{\partial \nu} d s=0
$$

Insbesondere folgt die Existenz von

$$
\lim _{\varepsilon \rightarrow 0} \int_{D_{\varepsilon, R}}|\operatorname{grad} u|^{2} d x=\operatorname{Re}\left(\bar{k}^{2}\right) \int_{|x| \leq R, x \notin D}|u|^{2} d x+\operatorname{Re} \int_{|x|=R} u \frac{\partial \bar{u}}{\partial \nu} d s .
$$

Als nächstes wird

$$
\lim _{\varepsilon \rightarrow 0} \operatorname{Im} \int_{\Gamma_{\varepsilon}} u \frac{\partial \bar{u}}{\partial \nu} d s=0
$$

gezeigt. Die Bildung des Imaginärteils von (4.6) ergibt

$$
0=\underbrace{\operatorname{Im}\left(\overline{k^{2}}\right) \int_{D_{\varepsilon, R}}|u|^{2} d x+\operatorname{Im} \int_{|x|=R} u \frac{\partial \bar{u}}{\partial \nu} d s}_{=: h(\varepsilon, R)}-\operatorname{Im} \int_{\Gamma_{\varepsilon}} u \frac{\partial \bar{u}}{\partial \nu} d s .
$$

Wir wissen $\lim _{\varepsilon \rightarrow 0} h(\varepsilon, R)$ existiert und für das Argument der Wellenzahl gilt $\arg (k) \in$ $[0, \pi / 2]$, also $\operatorname{Im}\left(\overline{k^{2}}\right) \leq 0$. Damit ist $h(\cdot, R)$ sowohl für $k \in \mathbb{R}^{+}$, als auch für $\operatorname{Im}(k)>$ 
$0, \operatorname{Re}(k) \geq 0$, monoton wachsend. Die Cauchy-Schwarzsche-Ungleichung

$$
|h(\varepsilon, R)| \leq M \sqrt{m+1} \sqrt{2 \pi \varepsilon}\left(\int_{\Gamma_{\varepsilon}}\left|\frac{\partial \bar{u}}{\partial \nu}\right|^{2} d s\right)^{1 / 2}
$$

liefert wegen

$$
\int_{\Gamma_{\varepsilon}}|\operatorname{grad} u|^{2} d s \geq \frac{|h(\varepsilon, R)|^{2}}{M^{2}(m+1) 2 \pi \varepsilon}
$$

sowohl für $h(0, R)>0$ als auch $h(0, R)<0$ einen Widerspruch zur Existenz von

$$
\lim _{\varepsilon \rightarrow 0} \int_{D_{\varepsilon, R}}|\operatorname{grad} u|^{2} d x,
$$

denn: Für hinreichend kleine $\varepsilon_{2}>\varepsilon_{1} \geq 0$ und geeignete Funktionen $\vartheta_{1, j}, \vartheta_{2, j}$, gilt

$$
\begin{aligned}
\int_{|x| \leq R, x \notin D}|\operatorname{grad} u|^{2} d x & \geq \sum_{j=0}^{m} \int_{\varepsilon_{1} \leq\left|x-x_{j}\right| \leq \varepsilon_{2}}|\operatorname{grad} u|^{2} d x \\
& =\int_{\varepsilon_{1}}^{\varepsilon_{2}} \sum_{j=0}^{m} \int_{\vartheta_{1, j}(r)}^{\vartheta_{2, j}(r)}|\operatorname{grad} u(r, \vartheta)|^{2} r d \vartheta d r \\
& \geq \int_{\varepsilon_{1}}^{\varepsilon_{2}} \frac{|h(r, R)|^{2}}{M^{2}(m+1) 2 \pi r} d r .
\end{aligned}
$$

Im Fall $h(0, R)>0$ setzen wir $\varepsilon_{1}=0$ und schätzen das letzte Integral nach unten ab durch

$$
\frac{|h(0, R)|^{2}}{M^{2}(m+1) 2 \pi} \int_{0}^{\varepsilon_{2}} \frac{d r}{r} \geq \infty .
$$

Im Fall $h(0, R)<0$ ergibt sich die gleiche Abschätzung unter Verwendung von $|h(r, R)|^{2}>$ $C>0$ auf $0 \leq r \leq \varepsilon_{2}$ und festem $R$. Also gilt $h(0, R)=0$.

Demnach verschwinden sowohl der Real- als auch Imaginärteil von

$$
\lim _{\varepsilon \rightarrow 0} \int_{\Gamma_{\varepsilon}} u \frac{\partial \bar{u}}{\partial \nu} d s .
$$

Die (bei festem $R$ ) bezüglich $\varepsilon$ gleichmäßige Beschränktheit der Terme

$$
\int_{D_{\varepsilon, R}}|u|^{2} d x, \int_{D_{\varepsilon, R}}|\operatorname{grad} u|^{2} d x
$$


und Existenz von

$$
\lim _{\varepsilon \rightarrow 0} \int_{\Gamma_{\varepsilon}} u \frac{\partial \bar{u}}{\partial \nu} d s=0
$$

erlaubt in (4.6) den Grenzübergang $\varepsilon \rightarrow 0$

$$
\int_{|x| \leq R, x \notin D}|\operatorname{grad} u|^{2} d x=\overline{k^{2}} \int_{|x| \leq R, x \notin D}|u|^{2} d x+\int_{|x|=R} u \frac{\partial \bar{u}}{\partial \nu} d s .
$$

Multiplikation mit $k$ und Imaginärteilbildung zusammen mit der (SAB) (4.3) ergibt

$$
\begin{aligned}
& 0 \stackrel{R \rightarrow \infty}{\longleftarrow} \int_{|x|=R}\left|\frac{\partial u}{\partial \nu}-i k u\right|^{2} d s \\
&=\int_{|x|=R}\left(\left|\frac{\partial u}{\partial \nu}\right|^{2}+|k u|^{2}\right) d s+2 \operatorname{Im}\left(k \int_{|x|=R} u \frac{\partial \bar{u}}{\partial \nu} d s\right) \\
&=\int_{|x|=R}\left(\left|\frac{\partial u}{\partial \nu}\right|^{2}+|k u|^{2}\right) d s+\underbrace{2 \operatorname{Im}(k)}_{\geq 0} \int_{|x| \leq R, x \notin D}|\operatorname{grad} u|^{2} d x \\
& \underbrace{-2 \operatorname{Im}(\bar{k})}_{\geq 0}|k|^{2} \int_{|x| \leq R, x \notin D}|u|^{2} d x \\
& \geq 0 .
\end{aligned}
$$

Für $k \in \mathbb{R}^{+}$folgt aus $\int_{|x|=R}|u|^{2} d s \rightarrow 0, R \rightarrow \infty$, die Behauptung $u=0$ aus dem RellichLemma [घ, Lemma 2.11], während sie für $\operatorname{Im}(k)>0$ unmittelbar aus $\int_{|x| \leq R, x \notin D}|u|^{2} d x \rightarrow$ $0, R \rightarrow \infty$, folgt.

Bemerkung 4.6 Die Beweisidee für Satz 4.5 ist den Arbeiten von Lewis-Wickham [4T] und Wickham [56] entnommen worden. Die erwähnten Autoren behandeln in ihren Artikeln jeweils ein äußeres Neumannproblem (in [56] zur Helmholtzgleichung) außerhalb eines Bogens. In Vogt [5.5, Abschnitt 5.2] ist auf ähnliche Weise ein Eindeutigkeitsbeweis zum Dirichletproblem bei der Elastizitätsgleichung geführt worden.

Bemerkung 4.7 Die Anwendung des Greenschen Satzes auf das Gebiet $D_{\varepsilon, R}$ und die Funktionen $u, \bar{u}$ ist noch zu rechtfertigen. Dazu ist das Gebiet $D$ in jeweils $\varepsilon / 2$-Umgebungen der Ecken $x_{0}, \ldots, x_{m}$ so zu modifizieren, dass das neue Gebiet $\tilde{D}$ einen $C^{2}$-glatten Rand besitzt und $D \subset \tilde{D}$ gilt. Betrachtet man nun das äußere Dirichletproblem mit Randwerten 
$\left.u\right|_{\partial \tilde{D}}$ bezüglich $\tilde{D}$, so folgt aus den Abbildungseigenschaften der Randintegral- und Potenzialoperatoren die $C^{1, \alpha}$-Regularität von $\tilde{u}$ bis in den Rand von $\tilde{D}$. Wegen der Eindeutigkeit stimmen $u$ und $\tilde{u}$ auf $\mathbb{R}^{2} \backslash \tilde{D}$ überein. Damit ist $u \in C^{2}\left(D_{\varepsilon, R}\right) \cap C^{1}\left(\overline{D_{\varepsilon, R}}\right)$.

Es ist gelegentlich günstig, auch für das adjungierte Problem die Eindeutigkeit sicherzustellen.

Satz 4.8 Für $\operatorname{Im}(k)>0$ besitzt das innere Neumannproblem (4.2) höchstens eine Lösung, während für $k \in \mathbb{R}^{+}$die Eindeutigkeit bis auf eine abzählbare Menge von Wellenzahlen, die sich nur im Unendlichen häufen, sichergestellt ist.

Beweis: Sei $u$ Lösung mit Randwerten $\frac{\partial u_{-}}{\partial \nu}=0$. Analog zum Beweis der Eindeutigkeit beim äußeren Dirichletproblem erklären wir für hinreichend kleine $\varepsilon$ Gebiete

$$
D_{\varepsilon}:=\left\{x \in D|| x-x_{j} \mid>\varepsilon \forall j=0, \ldots, m\right\}
$$

und Ränder

$$
\Gamma_{\varepsilon}:=\left\{x \in D \mid \exists j \in\{0, \ldots, m\} \text { mit }\left|x-x_{j}\right|=\varepsilon\right\} .
$$

Der Greensche Satz angewendet auf $D_{\varepsilon}, u, \bar{u}$ ergibt wegen der homogenen Randbedingung $u=0$ auf $\partial D$

$$
\int_{D_{\varepsilon}}\left(|\operatorname{grad} u|^{2}-\overline{k^{2}}|u|^{2}\right) d x=\int_{\Gamma_{\varepsilon}} u \frac{\partial \bar{u}}{\partial \nu} d s
$$

wobei die Normale $\nu(x), x \in \Gamma_{\varepsilon}$, in die Punkte $x_{0}, \ldots, x_{m}$ zeigt. Wiederum sichern Lemma 4.3 und 4.4 in (4.9) die Existenz der Grenzwerte für $\varepsilon \rightarrow 0$. Für $\operatorname{Im}(k)>0$ folgt die Behauptung aus

$$
\int_{D}|u|^{2} d x=0
$$

Für $k \in \mathbb{R}^{+}$folgt die Aussage aus der schwachen Formulierung des Randwertproblems. Mit einem Monotonieargument wird das Problem reduziert auf $C^{2}$-glatte Gebiete. Der dazugehörige Operator ist kompakt und besitzt daher nur abzählbar viele Eigenwerte, die sich höchstens bei Null häufen können. Für eine detaillierte Darstellung siehe [9].

\subsection{Potenziale und Sprungbeziehungen}

Definition 4.9 Für $\varphi \in C(\partial D)$ heißt

$$
u(x)=\int_{\partial D} \Phi_{k}(x, y) \varphi(y) d s(y), x \in \mathbb{R}^{2} \backslash \partial D,
$$

Einfachschichtpotenzial mit Dichte $\varphi$. 
Definition 4.10 Für $\varphi \in C(\partial D)$ heißt

$$
v(x)=\int_{\partial D} \frac{\partial \Phi_{k}(x, y)}{\partial \nu(y)} \varphi(y) d s(y), x \in \mathbb{R}^{2} \backslash \partial D,
$$

Doppelschichtpotenzial mit Dichte $\varphi$.

\section{Satz 4.11}

i) Das Einfachschichtpotenzial u mit stetiger Dichte $\varphi$ ist stetig in ganz $\mathbb{R}^{2}$.

ii) Für die Normalableitung des Einfachschichtpotenzials mit stetiger Dichte $\varphi \in C(\partial D)$ gilt

$$
\frac{\partial u_{ \pm}(x)}{\partial \nu(x)}=\int_{\partial D} \frac{\partial \Phi_{k}(x, y)}{\partial \nu(x)} \varphi(y) d s(y) \mp \frac{1}{2} \varphi(x), x \in \partial D \backslash\left\{x_{0}, \ldots, x_{m}\right\} .
$$

Beweis: zu i) Da $\Phi_{0}$ und $\Phi_{k}$ für $|x-y| \rightarrow 0$ das gleiche singuläre Verhalten aufweisen, folgt die Behauptung aus Hackbusch [18, Satz 8.1.7].

zu ii) Für $C^{2}$-glatte Ränder sind die Beweise in [6, Theorem 2.19] im 3-dimensionalen Fall und in [5], Satz 2.29] im 2-dimensionalen Fall ausgeführt worden. Da Sprungbeziehungen lokale Eigenschaften sind, bleiben die Resultate für stückweise glatte Ränder richtig.

\section{Satz 4.12}

i) Das Doppelschichtpotenzial $v$ mit stetiger Dichte $\varphi$ lässt sich stetig von $D$ nach $\bar{D}$ beziehungsweise von $\mathbb{R}^{2} \backslash \bar{D}$ nach $\mathbb{R}^{2} \backslash D$ fortsetzen mit den Randwerten

$$
v_{ \pm}(x)=\int_{\partial D} \frac{\partial \Phi_{k}(x, y)}{\partial \nu(y)} \varphi(y) d s(y)+\frac{\beta(x)-\pi \pm \pi}{2 \pi} \varphi(x), x \in \partial D .
$$

ii) Für die Normalableitung des Doppelschichtpotenzials mit stetiger Dichte $\varphi \in C(\partial D)$ gilt

$$
\frac{\partial v_{+}(x)}{\partial \nu(x)}=\frac{\partial v_{-}(x)}{\partial \nu(x)}, x \in \partial D \backslash\left\{x_{0}, \ldots, x_{m}\right\}
$$

Beweis: zu i) Für $x \in \partial D \backslash\left\{x_{0}, \ldots, x_{m}\right\}$ verläuft der Beweis wie in [5], Satz 2.24]. Nach Abspalten der konstanten Dichte erhält man (siehe [2, Lemma 3.2]) zunächst

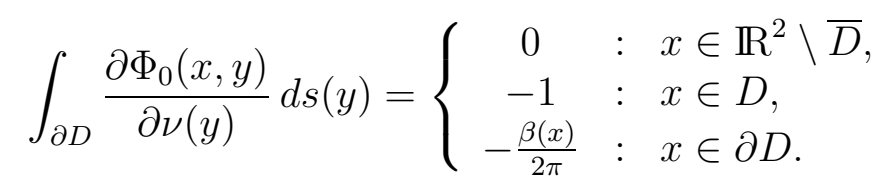


Wie im Beweis von [6, Theorem 2.13] zeigt man die Stetigkeit von

$$
\int_{\partial D} \frac{\partial \Phi_{0}(x, y)}{\partial \nu(y)}\left[\varphi(y)-\varphi\left(x_{0}\right)\right] d s(y), x \in \mathbb{R}^{2}
$$

in einer (einseitigen) Umgebung von $x_{0}$. Zusammen mit (4.10) und dem gleichen singulären Verhalten von $\Phi_{0}$ und $\Phi_{k}$ ergibt sich die Behauptung.

zu ii) Für $C^{2}$-glatte Ränder sind die Beweise in [6, Theorem 2.21] im 3-dimensionalen Fall und in [5], Satz 2.32] im 2-dimensionalen Fall ausgeführt worden. Wiederum bleibt die Aussage richtig, denn Sprungbeziehungen sind lokale Eigenschaften.

\subsection{Zur Existenz beim direkten Dirichletproblem}

Der Existenzbeweis soll zur Vereinfachung der Darstellung nur für den Fall einer Ecke geführt werden. Der Greensche Darstellungssatz motiviert den Potenzialansatz

$$
u(x)=\int_{\partial D}\left[\frac{\partial \Phi_{k}}{\partial \nu(y)}(x, y)-i \eta \Phi_{k}(x, y)\right] \varphi(y) d s(y), x \notin \partial D,
$$

mit Kopplungsparameter $\eta>0$ und Dichte $\varphi \in C(\partial D)$.

Satz 4.13 Das Gebiet D erfülle die angegebenen Voraussetzungen mit $m=0$. Weiterhin sei $f \in C(\partial D)$. Dann gilt: Für jede Lösung $\varphi \in C(\partial D)$ der Integralgleichung

$$
\begin{aligned}
2 f(x)= & \varphi(x)+2 \int_{\partial D}\left[\frac{\partial \Phi_{k}}{\partial \nu(y)}(x, y)-i \eta \Phi_{k}(x, y)\right]\left(\varphi(y)-\varphi\left(x_{0}\right)\right) d s(y) \\
& +\varphi\left(x_{0}\right) \gamma(x), x \in \partial D
\end{aligned}
$$

mit der stetigen Funktion $\gamma$ gemäß

$$
\gamma(x)=2 \int_{\partial D}\left(\frac{\partial \Phi_{k}}{\partial \nu(y)}(x, y)-\frac{\partial \Phi_{0}}{\partial \nu(y)}(x, y)-i \eta \Phi_{k}(x, y)\right) d s(y)-1
$$

wird durch

$$
u(x)=\int_{\partial D}\left[\frac{\partial \Phi_{k}}{\partial \nu(y)}(x, y)-i \eta \Phi_{k}(x, y)\right] \varphi(y) d s(y), x \notin \bar{D},
$$

eine Lösung des äußeren Dirichletproblems (4.1) - (4.3) gegeben. 
Beweis: Die durch (4.13) definierte Funktion $u$ erfüllt die Sommerfeldsche Ausstrahlungsbedingung (weil $\Phi_{k}$ sie erfüllt), löst die Helmholtzgleichung in $\mathbb{R}^{2} \backslash \bar{D}$ und ist stetig bis in den Rand $\partial D$. Weil

$$
\int_{\partial D} \frac{\partial \Phi_{0}}{\partial \nu(y)}(x, y) d s(y)=0, x \in \mathbb{R}^{2} \backslash \bar{D}
$$

liefern die Sprungbeziehungen für Einfach- (Satz 4.11) und Doppelschichtpotenzial (Satz 4.12) angewendet auf

$$
\begin{aligned}
u(x)= & \int_{\partial D}\left[\frac{\partial \Phi_{k}}{\partial \nu(y)}(x, y)-i \eta \Phi_{k}(x, y)\right]\left(\varphi(y)-\varphi\left(x_{0}\right)\right) d s(y) \\
& +\varphi\left(x_{0}\right) \int_{\partial D}\left[\frac{\partial \Phi_{k}}{\partial \nu(y)}(x, y)-\frac{\partial \Phi_{0}}{\partial \nu(y)}(x, y)-i \eta \Phi_{k}(x, y)\right] d s(y), x \in \mathbb{R}^{2} \backslash \bar{D}
\end{aligned}
$$

die Gleichung

$$
\begin{aligned}
2 u_{+}(x)= & \varphi(x)+2 \int_{\partial D}\left[\frac{\partial \Phi_{k}}{\partial \nu(y)}(x, y)-i \eta \Phi_{k}(x, y)\right]\left(\varphi(y)-\varphi\left(x_{0}\right)\right) d s(y) \\
& +\varphi\left(x_{0}\right) \gamma(x)=2 f(x), x \in \partial D
\end{aligned}
$$

Daher ist die Randbedingung $u=f$ ebenfalls erfüllt.

Satz 4.14 Für jedes $f \in C(\partial D)$ hat die Integralgleichung (4.19) genau eine Lösung $\varphi \in C(\partial D)$.

Beweis: Sei $\varphi \in C(\partial D)$ eine Lösung der homogenen Gleichung. Dann erfüllt nach Satz 4.13 das durch (4.11) definierte $u$ das homogene äußere Dirichlet-Problem. Nach Satz 4.5 verschwindet $u$ in $\mathbb{R}^{2} \backslash D$. Die Sprungbeziehungen (siehe Sätze 4.11 und 4.12) liefern

$$
-u_{-}=\varphi,-\frac{\partial u_{-}}{\partial \nu}=i \eta \varphi \text { auf } \partial D \backslash\left\{x_{0}\right\}
$$

Der erste Greensche Satz, dessen Anwendung wie im Beweis von Satz 4.5 sichergestellt werden kann, ergibt

$$
\begin{aligned}
\int_{D}\left\{|\operatorname{grad} u(x)|^{2}-k^{2}|u(x)|^{2}\right\} d x & =\int_{\partial D} \bar{u}_{-}(x) \frac{\partial u_{-}(x)}{\partial \nu(x)} d s \\
& =i \eta \int_{\partial D}|\varphi(x)|^{2} d s
\end{aligned}
$$


Durch Imaginärteilbildung und Beachtung von $\operatorname{Im}\left(k^{2}\right) \geq 0, \eta>0$ ergibt sich $\varphi=0$.

Es genügt nun nachzuweisen, dass der Randintegraloperator in (4.12) darstellbar ist als Summe aus einem kompakten und einem beschränkt invertierbaren Operator, denn dann liefert die Riesz-Theorie die Behauptung. Für den Nachweis, dass sich $A$ mit

$$
\begin{aligned}
(A \varphi)(x) & =2 \int_{\partial D}\left[\frac{\partial \Phi_{k}}{\partial \nu(y)}(x, y)-i \eta \Phi_{k}(x, y)\right]\left(\varphi(y)-\varphi\left(x_{0}\right)\right) d s(y) \\
& +\varphi\left(x_{0}\right)\left(2 \int_{\partial D}\left[\frac{\partial \Phi_{k}}{\partial \nu(y)}(x, y)-\frac{\partial \Phi_{0}}{\partial \nu(y)}(x, y)-i \eta \Phi_{k}(x, y)\right] d s(y)-1\right), x \in \partial D,
\end{aligned}
$$

mit Hilfe einer geeigneten Abschneidefunktion aufspalten lässt in $A=A_{1}+A_{2}$, mit einem kompakten Operator $A_{1}: C(\partial D) \rightarrow C(\partial D)$ und einem Operator $A_{2}$ mit Norm $\left\|A_{2}\right\|<1$, sei auf [2, Sätze 3.12 - 3.14] verwiesen.

\subsection{Der Fernfeldoperator}

Satz 4.15 Eine ausstrahlende Lösung u der Helmholtzgleichung hat das asymptotische Verhalten

$$
u(x)=\frac{e^{i k|x|}}{\sqrt{|x|}}\left\{u_{\infty}(\hat{x})+O\left(\frac{1}{|x|}\right)\right\},|x| \rightarrow \infty .
$$

Falls bis auf endlich viele Punkte der Grenzwert

$$
\frac{\partial u}{\partial \nu}(x)=\lim _{h \rightarrow+0} \nu(x) \cdot \operatorname{grad} u(x+h \nu(x)), x \in \partial D,
$$

existiert, und zwar gleichmäßig auf $\partial D$, so gilt

$$
u_{\infty}(\hat{x})=\frac{e^{i \frac{\pi}{4}}}{\sqrt{8 \pi k}} \int_{\partial D}\left\{u(y) \frac{\partial e^{-i k \hat{x} \cdot y}}{\partial \nu(y)}-\frac{\partial u}{\partial \nu}(y) e^{-i k \hat{x} \cdot y}\right\} d s(y)
$$

wobei $\hat{x}=x /|x|$ bezeichnet.

Beweis: Vergleiche [[, Theorem 2.5].

\section{Definition 4.16 (Direktes Streuproblem)}

Für eine ebene einfallende Welle $u^{i}(x, d)=e^{i k x \cdot d}$ mit Einfallsrichtung $d \in \mathbb{R}^{2},|d|=1$, 
wird das gestreute Feld $u^{s} \in C^{2}\left(\mathbb{R}^{2} \backslash \bar{D}\right) \cap C\left(\mathbb{R}^{2} \backslash D\right)$ gesucht (beziehungsweise das Fernfeld von $u^{s}+u^{i}$ ) als Lösung der Helmholtzgleichung

$$
\triangle u^{s}+k^{2} u^{s}=0 \quad \text { in } \mathbb{R}^{2} \backslash \bar{D},
$$

das die Randbedingung (für schallweiche Hindernisse)

$$
u^{s}=-u^{i} \quad \text { auf } \partial D
$$

und die Sommerfeldsche Ausstrahlungsbedingung (SAB)

$$
\lim _{r \rightarrow \infty} r^{1 / 2}\left(\frac{\partial u^{s}}{\partial r}-i k u^{s}\right)=0, r=|x|,
$$

gleichmäßig für alle Richtungen $\hat{x}=\frac{x}{|x|}$ erfüllt.

Insbesondere folgt aus dem asymptotischen Verhalten (2.4) der Hankelfunktion für das Fernfeld $u_{\infty}$ von

$$
u^{s}(x)=\int_{\partial D}\left[\frac{\partial \Phi_{k}}{\partial \nu(y)}(x, y)-i \eta \Phi_{k}(x, y)\right] \varphi(y) d s(y), x \notin \bar{D},
$$

die Darstellung

$$
u_{\infty}(\hat{x})=\frac{e^{-i \frac{\pi}{4}}}{\sqrt{8 \pi k}} \int_{\partial D}\{k \nu(y) \cdot \hat{x}+\eta\} e^{-i k \hat{x} \cdot y} \varphi(y) d s(y),|\hat{x}|=1 .
$$

Der Operator $\mathcal{F}$, der einem zulässigen Rand $\partial D$ das Fernfeld $u_{\infty}$ zuordnet, heißt Fernfeldoperator. Beim direkten Problem sind folglich (bei fester Wellenzahl $k$ und verschiedenen Einfallsrichtungen $d$ ) bei gegebener Parametrisierung $z$ die Fernfelder $\mathcal{F}(z)$ zu berechnen. Beim inversen Problem wird versucht, aus gemessenen Fernfeldern zu verschiedenen einfallenden Wellen die Parametrisierung und damit den Rand des Gebietes zu rekonstruieren. 


\subsection{Numerische Ergebnisse}

Zunächst soll die numerische Implementierung beschrieben werden.

In diesem Abschnitt beschränken wir uns wieder auf zulässige Gebiete $D$ mit nur einer Ecke. Aus dem Abschnitt 4.4 ist bekannt, dass die Lösung des äußeren Dirichletproblems mit Randwerten $f \in C(\partial D)$ gegeben ist durch

$$
u(x)=\int_{\partial D}\left[\frac{\partial \Phi_{k}}{\partial \nu(y)}(x, y)-i \eta \Phi_{k}(x, y)\right] \varphi(y) d s(y), x \notin \bar{D},
$$

mit der eindeutig bestimmten Lösung $\varphi \in C(\partial D)$ der Randintegralgleichung

$$
\begin{aligned}
2 f(x)= & \varphi(x)+2 \int_{\partial D}\left[\frac{\partial \Phi_{k}}{\partial \nu(y)}(x, y)-i \eta \Phi_{k}(x, y)\right]\left(\varphi(y)-\varphi\left(x_{0}\right)\right) d s(y) \\
& +\varphi\left(x_{0}\right) \gamma(x), \quad x \in \partial D .
\end{aligned}
$$

Dabei ist die auf $\partial D$ stetige Funktion $\gamma$ gegeben durch

$$
\gamma(x)=2 \int_{\partial D}\left(\frac{\partial \Phi_{k}}{\partial \nu(y)}(x, y)-\frac{\partial \Phi_{0}}{\partial \nu(y)}(x, y)-i \eta \Phi_{k}(x, y)\right) d s(y)-1 .
$$

Wir parametrisieren den Rand von $D$ mit einer Funktion $z \in C_{2 \pi}(\mathbb{R}, \partial D)$ mit den Eigenschaften:

- $\left.z\right|_{[0,2 \pi]} \in C^{2}\left([0,2 \pi], \mathbb{R}^{2}\right)$,

- $\left.z\right|_{[0,2 \pi)}$ ist injektiv und linksorientiert,

- $z(0)=x_{0},\left|z^{\prime}(t)\right|>0 \forall t \in \mathbb{R}$ und

- $z^{\prime}(0) \cdot z^{\prime}(2 \pi) \neq-\left|z^{\prime}(0)\right|\left|z^{\prime}(2 \pi)\right|$.

Die letzte Bedingung stellt sicher, dass $\partial D$ keine Spitzen besitzt. Wegen

$$
2 \frac{\partial \Phi_{k}}{\partial \nu(y)}(x, y)=\frac{i k}{2} H_{1}^{(1)}(k|x-y|) \frac{\nu(y) \cdot(x-y)}{|x-y|}
$$


ergibt sich für $\tilde{f}(t)=2 f(z(t)), \tilde{\varphi}(t)=\varphi(z(t))$

$$
\begin{aligned}
\tilde{f}(t)= & \tilde{\varphi}(t)+\int_{0}^{2 \pi}\left\{L_{k}(t, \tau)-i \eta M_{k}(t, \tau)\right\}[\tilde{\varphi}(\tau)-\tilde{\varphi}(0)] d \tau \\
& +\tilde{\varphi}(0)\left[\int_{0}^{2 \pi}\left\{\left(L_{k}-L_{0}\right)(t, \tau)-i \eta M_{k}(t, \tau)\right\} d \tau-1\right], 0 \leq t \leq 2 \pi .
\end{aligned}
$$

Der Einfachheit halber werden zukünftig statt $\tilde{f}$ und $\tilde{\varphi}$ einfach $f$ und $\varphi$ geschrieben. Die Kerne $M_{k}, L_{k}, L_{0}$ sind gegeben durch

$$
\begin{aligned}
M_{k}(t, \tau) & =\frac{i}{2} H_{0}^{(1)}(k|z(t)-z(\tau)|)\left|z^{\prime}(\tau)\right| \\
L_{k}(t, \tau) & =\frac{i k}{2} H_{1}^{(1)}(k|z(t)-z(\tau)|) \frac{\left(z_{2}^{\prime}(\tau),-z_{1}^{\prime}(\tau)\right) \cdot[z(t)-z(\tau)]}{|z(t)-z(\tau)|}, \\
L_{0}(t, \tau) & =\frac{1}{\pi} \frac{\left(z_{2}^{\prime}(\tau),-z_{1}^{\prime}(\tau)\right) \cdot[z(t)-z(\tau)]}{|z(t)-z(\tau)|^{2}} .
\end{aligned}
$$

Die Abspaltung der logarithmischen Singularität führt zu

$$
\begin{aligned}
M_{k}(t, \tau) & =M_{k}^{(1)}(t, \tau) \ln \left(4 \sin ^{2} \frac{t-\tau}{2}\right)+M_{k}^{(2)}(t, \tau), \\
L_{k}(t, \tau) & =L_{k}^{(1)}(t, \tau) \ln \left(4 \sin ^{2} \frac{t-\tau}{2}\right)+L_{k}^{(2)}(t, \tau)
\end{aligned}
$$

mit den Kernen

$$
\begin{aligned}
M_{k}^{(1)}(t, \tau) & :=-\frac{1}{2 \pi} J_{0}(k|z(t)-z(\tau)|)\left|z^{\prime}(\tau)\right| \\
M_{k}^{(2)}(t, \tau) & :=M_{k}(t, \tau)-M_{k}^{(1)}(t, \tau) \ln \left(4 \sin ^{2} \frac{t-\tau}{2}\right) \\
L_{k}^{(1)}(t, \tau) & :=-\frac{k}{2 \pi} J_{1}(k|z(t)-z(\tau)|) \frac{\left(z_{2}^{\prime}(\tau),-z_{1}^{\prime}(\tau)\right) \cdot[z(t)-z(\tau)]}{|z(t)-z(\tau)|} \\
L_{k}^{(2)}(t, \tau) & :=L_{k}(t, \tau)-L_{k}^{(1)}(t, \tau) \ln \left(4 \sin ^{2} \frac{t-\tau}{2}\right) .
\end{aligned}
$$

Aus den Definitionen von Bessel- (2.2) und Neumannfunktionen (2.3) ist bei $C^{2}$-glatten Rändern $\partial D \backslash\left\{x_{0}\right\}$ für die Diagonalterme

$$
M_{k}^{(1)}(t, t)=-\frac{1}{2 \pi}\left|z^{\prime}(t)\right|,
$$




$$
\begin{aligned}
M_{k}^{(2)}(t, t) & =\left(\frac{i}{2}-\frac{1}{\pi}\left\{C_{E}+\ln \left(\frac{k}{2}\left|z^{\prime}(t)\right|\right)\right\}\right)\left|z^{\prime}(t)\right|, \\
L_{k}^{(1)}(t, t) & =0 \\
L_{k}^{(2)}(t, t) & =\frac{1}{2 \pi} \frac{\left(z_{2}^{\prime}(t),-z_{1}^{\prime}(t)\right) \cdot z^{\prime \prime}(t)}{\left|z^{\prime}(t)\right|^{2}}
\end{aligned}
$$

abzulesen. Falls $\partial D \backslash\left\{x_{0}\right\}$ die Randglätte $C^{2 q+3}$ besitzt, so sind $M_{k}^{(1)}, M_{k}^{(2)}, L_{0}, L_{k}^{(1)}$ und $L_{k}^{(2)}$ bis auf die Punkte $(0,2 \pi),(2 \pi, 0)$ aus $C^{2 q+1}([0,2 \pi] \times[0,2 \pi])$.

Im Fall des Streuproblems bei einfallender ebener Welle $u^{i}(x, d)$ lauten die Komponenten $f_{l}, l=0, \ldots, 2 n-1$, der rechten Seite des Gleichungssystems (3.20)

$$
f_{l}=-2 e^{i k z\left(s_{l}^{(n)}\right) \cdot d}
$$

Für die Kerne $K_{A}, K_{B}, K_{C}$ in (3.4) - (3.6) gilt

$$
\begin{array}{cl}
\left(K_{A}+K_{B}\right)(t, \tau) & =-L_{k}^{(2)}(t, \tau)+i \eta M_{k}^{(2)}(t, \tau), \\
K_{C}(t, \tau) & =-L_{k}^{(1)}(t, \tau)+i \eta M_{k}^{(1)}(t, \tau) .
\end{array}
$$

Auf einen detaillierten Nachweis dafür, dass sich $L_{k}^{(2)}$ in den Ecken tatsächlich wie ein Mellin-Kern verhält, wird an dieser Stelle verzichtet, denn dieser ist (als Spezialfall) im zweiten Teil des Beweises von Satz 5.10 enthalten. Damit ist die Integralgleichung (4.21) von der Form (3.1) aus Kapitel 3 .

Durch Lösen des resultierenden Gleichungssystems (3.20) ergeben sich Näherungswerte $\varphi_{j}^{(n)}$ für die exakten Werte $\varphi(z(w(j \pi / n))), j=0, \ldots, 2 n-1$. Anwendung der Quadraturformel (3.8) zur approximativen Auswertung von (4.19) ergibt

$$
\begin{aligned}
u_{\infty}^{s}(\hat{x}) \approx & \frac{e^{-i \frac{\pi}{4}}}{\sqrt{8 \pi k}} \frac{\pi}{n} \sum_{j=0}^{2 n-1}\left(k\left(\hat{x}_{1} z_{2}^{\prime}\left(s_{j}^{(n)}\right)-\hat{x}_{2} z_{1}^{\prime}\left(s_{j}^{(n)}\right)\right)+\eta\left|z^{\prime}\left(s_{j}^{(n)}\right)\right|\right) \varphi_{j}^{(n)} \times \\
& \times \alpha_{j}^{(n)} e^{-i k\left(\hat{x}_{1} z_{1}\left(s_{j}^{(n)}\right)+\hat{x}_{2} z_{2}\left(s_{j}^{(n)}\right)\right)} \text { für } \hat{x} \in \Omega .
\end{aligned}
$$

Der Summand für $j=0$ in (4.32) tritt nur bei glatten Rändern (mit der Substitution $w(t)=t$ ) auf, weil für die betrachteten Substitutionen $w$ gemäß Definition 3.3 der Term $\alpha_{0}^{(n)}=w^{\prime}(0)$ verschwindet. 
Beispiel 4.17 Es wird ein konvexes Gebiet in Form eines Tropfens betrachtet, das in $(0,0)^{t}$ eine Ecke mit Innenwinkel $\frac{\pi}{2}$ besitzt. Der Rand wird durch

$$
z(t)=\left(2 \sin \frac{t}{2},-\sin (t)\right), 0 \leq t \leq 2 \pi,
$$

parametrisiert.

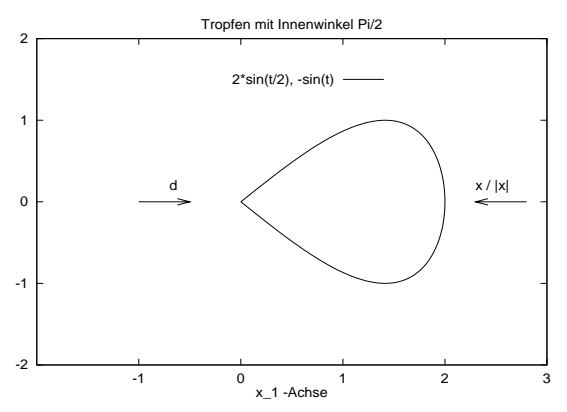

In Tabelle 4.7 sind bei einfallender ebener Welle $u^{i}(x, d)=e^{i k x \cdot d}$ das Fernfeld $u_{\infty}$ für die Beobachtungsrichtungen $\hat{x}=d=(1,0)^{t}$ und $\hat{x}=-d$ dargestellt. Dabei wurden Kopplungsparameter $\eta$ und Wellenzahl $k$ jeweils 1 gesetzt. Beim Aufstellen des Gleichungssystems zur Ermittlung von Näherungswerten $\varphi_{j} \approx \varphi\left(z\left(s_{j}^{(n)}\right)\right.$ wurde die Substitution nach Kreß verwendet.

Abbildung 4.1: Tropfen mit Innenwinkel $\frac{\pi}{2}$

Beispiel 4.18 Es wird ein nichtkonvexes Gebiet in Form eines Schmetterlings betrachtet, das in $(-1 / 2,0)^{t}$ eine Ecke mit Innenwinkel $\frac{3 \pi}{2}$ besitzt. Der Rand wird durch

$$
z(t)=\left(-\frac{2}{3} \sin \frac{3}{2} t-\frac{1}{2},-\sin (t)\right), 0 \leq t \leq 2 \pi,
$$

parametrisiert.

Beispiel 4.19 Mit der Parametrisierung

$$
z(t)=\left(\frac{2}{\sqrt{3}} \sin \frac{t}{2}-1,-\sin (t)\right), 0 \leq t \leq 2 \pi,
$$

wird ein weiteres konvexes tropfenförmiges Gebiet betrachtet. Im Punkt $(-1,0)^{t}$ liegt eine Ecke mit Innenwinkel $\frac{2 \pi}{3}$ vor. 


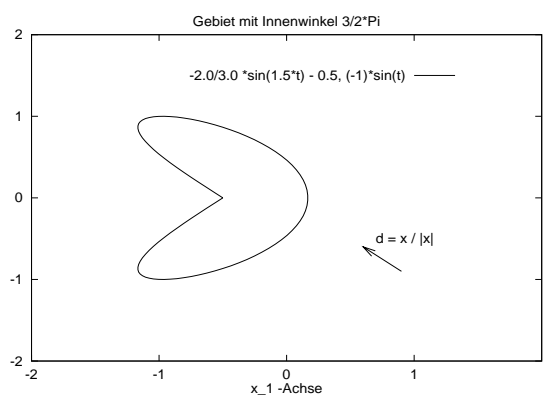

In Tabelle 4.2 sind bei einfallender ebener Welle $u^{i}(x, d)=e^{i k x \cdot d}$ das Fernfeld $u_{\infty}$ für die Beobachtungsrichtung $\hat{x}=d=1 / \sqrt{2}(-1,1)^{t}$ dargestellt. Dabei wurden Kopplungsparameter $\eta$ und Wellenzahl $k$ jeweils 5 gesetzt. Als Substitutionen wurden die trigonometrische nach Sidi beziehungsweise die rationale nach Kreß verwendet.

Abbildung 4.2: Schmetterling mit Innenwinkel $\frac{3}{2} \pi$

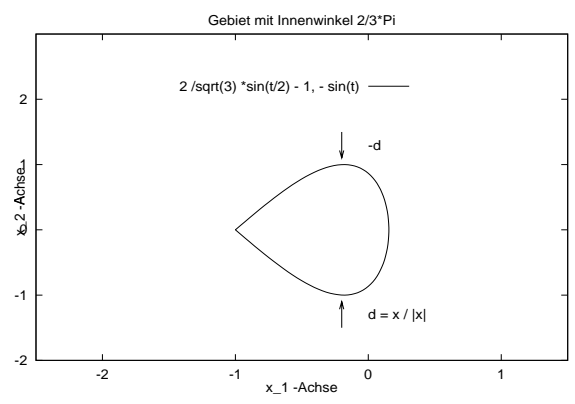

In Tabelle 4.3 sind bei einfallender ebener Welle $u^{i}(x, d)=e^{i k x \cdot d}$ das Fernfeld $u_{\infty}$ für die Beobachtungsrichtungen $\hat{x}=d=(0,+1)^{t}$ und $\hat{x}=-d$ dargestellt. Dabei wurden Kopplungsparameter $\eta$ und Wellenzahl $k$ jeweils 2 gesetzt. Als Substitution wurde die polynomiale nach Korobov verwendet.

Abbildung 4.3: Tropfen mit Innenwinkel $\frac{2}{3} \pi$

Den Tabellen ist zu entnehmen, dass sich die erwartete schnelle Konvergenz einstellt. Bei $n=32$ ist, von wenigen Ausnahmen abgesehen, bereits die vierte Nachkommastelle exakt. $\mathrm{Zu}$ diesem Zeitpunkt beträgt der relative Fehler rund $10^{-4}$.

Um die Korrektheit des Algorithmus' zu testen, wird dieser auf Randwertprobleme angewendet, bei denen die Lösung bekannt ist. Eine Möglichkeit besteht darin, für Gebiete $D$, die den Nullpunkt enthalten, die Hankelfunktion $H_{0}^{(1)}$ auf dem Rand vorzugeben. Anschließend ist ein Vergleich der numerischen Werte mit dem exakten Fernfeld

$$
u_{\infty}(\hat{x})=\sqrt{\frac{2}{\pi k}} e^{-i \frac{\pi}{4}}
$$

möglich. Auf eine explizite Angabe von Zahlenwerten bei diesen Tests wurde hier verzichtet. 
Tabelle 4.1: Tabelle zu Beispiel 4.17.

Parametrisierung $z(t)=\left(2 \sin \frac{t}{2},-\sin t\right)$, Substitution $w$ mit kubischem $v$ von Kreß, $d=(1,0)^{t}$ und $\eta=k=1$.

\begin{tabular}{|c|r|c|c|c|c|}
\hline \hline \multicolumn{1}{|c|}{$p$} & $n$ & $\operatorname{Re} u_{\infty}(d)$ & $\operatorname{Im} u_{\infty}(d)$ & $\operatorname{Re} u_{\infty}(-d)$ & $\operatorname{Im} u_{\infty}(-d)$ \\
\hline & 8 & -1.2854092987 & +0.3069295365 & -0.5299830219 & -0.4116485500 \\
4 & 16 & -1.2854914279 & +0.3068663543 & -0.5302057209 & -0.4109821566 \\
& 32 & -1.2854934933 & +0.3068662265 & -0.5302102214 & -0.4109640354 \\
& 64 & -1.2854935289 & +0.3068662733 & -0.5302102602 & -0.4109636617 \\
& 128 & -1.2854935289 & +0.3068662774 & -0.5302102576 & -0.4109636563 \\
\hline & 8 & -1.2854395462 & +0.3069903503 & -0.5297463540 & -0.4120187868 \\
6 & 16 & -1.2854916555 & +0.3068673994 & -0.5302051881 & -0.4109787775 \\
& 32 & -1.2854935188 & +0.3068662834 & -0.5302102343 & -0.4109637370 \\
& 64 & -1.2854935288 & +0.3068662778 & -0.5302102572 & -0.4109636567 \\
& 128 & -1.2854935288 & +0.3068662777 & -0.5302102573 & -0.4109636564 \\
\hline & 8 & -1.2855031044 & +0.3067855340 & -0.5291350715 & -0.4118560099 \\
& 16 & -1.2854906812 & +0.3068680469 & -0.5302019695 & -0.4109864359 \\
& 32 & -1.2854935212 & +0.3068662820 & -0.5302102389 & -0.4109637183 \\
& 64 & -1.2854935288 & +0.3068662777 & -0.5302102573 & -0.4109636565 \\
& 128 & -1.2854935288 & +0.3068662777 & -0.5302102573 & -0.4109636564 \\
\hline
\end{tabular}


Tabelle 4.2: Tabelle zu Beispiel 4.18.

Parametrisierung: $z(t)=\left(-\frac{2}{3} \sin \frac{3}{2} t-\frac{1}{2},-\sin t\right)$

Substitution: $\sin ^{p}$ nach Sidi im Vergleich mit $w$ nach Kreß

Einfallsrichtung $d=1 / \sqrt{2}(-1,1)^{t}$ und $\eta=k=5$.

\begin{tabular}{|c|r|c|c|c|c|}
\hline \hline \multirow{4}{*}{$p$} & $n$ & $\operatorname{Re} u_{\infty}(d)$ & $\operatorname{Im} u_{\infty}(d)$ & $\operatorname{Re} u_{\infty}(d)$ & $\operatorname{Im} u_{\infty}(d)$ \\
\hline \multirow{4}{*}{4} & 8 & -1.4072330285 & +1.0508604210 & -1.4346817212 & +1.0745565340 \\
& 16 & -1.5547401666 & +0.9955621878 & -1.5717267351 & +1.0036287382 \\
& 32 & -1.5888757116 & +1.0133676423 & -1.5889141580 & +1.0133688113 \\
& 64 & -1.5889159254 & +1.0133686822 & -1.5889159251 & +1.0133686816 \\
& 128 & -1.5889159232 & +1.0133686783 & -1.5889159231 & +1.0133686783 \\
\hline \multirow{6}{*}{6} & 8 & -1.3183668788 & +1.0288005117 & -1.4163739389 & +1.0669492206 \\
& 16 & -1.5081680918 & +1.0013490961 & -1.5669699572 & +1.0006366873 \\
& 32 & -1.5878331045 & +1.0131264006 & -1.5889116095 & +1.0133685759 \\
& 64 & -1.5889159230 & +1.0133686780 & -1.5889159230 & +1.0133686780 \\
& 128 & -1.5889159230 & +1.0133686780 & -1.5889159230 & +1.0133686780 \\
\hline \multirow{6}{*}{8} & 8 & -1.3183365112 & +1.0188463707 & -1.4113929424 & +1.0763835530 \\
& 16 & -1.4785434564 & +1.0238904333 & -1.5618664155 & +0.9966208876 \\
& 32 & -1.5840429983 & +1.0114688763 & -1.5889052456 & +1.0133676473 \\
& 64 & -1.5889159104 & +1.0133686802 & -1.5889159230 & +1.0133686780 \\
& 128 & -1.5889159230 & +1.0133686780 & -1.5889159230 & +1.0133686780 \\
\hline
\end{tabular}


Tabelle 4.3: Tabelle zu Beispiel 4.19.

Parametrisierung: $z(t)=\left(\frac{2}{\sqrt{3}} \sin \frac{t}{2}-1,-\sin t\right)$

Substitution: polynomial nach Korobov

Einfallsrichtung $d=(0,+1)^{t}$ und $\eta=k=2$.

\begin{tabular}{|c|r|c|c|c|c|}
\hline \hline \multirow{4}{*}{$p$} & $n$ & $\operatorname{Re} u_{\infty}(d)$ & $\operatorname{Im} u_{\infty}(d)$ & $\operatorname{Re} u_{\infty}(-d)$ & $\operatorname{Im} u_{\infty}(-d)$ \\
\hline \multirow{4}{*}{4} & 8 & -1.1771133343 & +0.2647983899 & +0.4036409437 & -0.1740620044 \\
& 16 & -1.1810605251 & +0.2650020399 & +0.4128910217 & -0.1616384258 \\
& 32 & -1.1810599660 & +0.2650032202 & +0.4128923494 & -0.1616367680 \\
& 64 & -1.1810598834 & +0.2650033097 & +0.4128924320 & -0.1616366785 \\
& 128 & -1.1810598782 & +0.2650033150 & +0.4128924373 & -0.1616366731 \\
\hline \multirow{6}{*}{6} & 8 & -1.1674860269 & +0.2599372003 & +0.3650325653 & -0.2088868287 \\
& 16 & -1.1810403140 & +0.2649968821 & +0.4128818530 & -0.1616823652 \\
& 32 & -1.1810598774 & +0.2650033152 & +0.4128924380 & -0.1616366729 \\
& 64 & -1.1810598778 & +0.2650033154 & +0.4128924376 & -0.1616366728 \\
& 128 & -1.1810598778 & +0.2650033154 & +0.4128924376 & -0.1616366728 \\
\hline \multirow{6}{*}{8} & 8 & -1.1474431832 & +0.2702118104 & +0.3042351489 & -0.2260422069 \\
& 16 & -1.1808743769 & +0.2649685379 & +0.4127626480 & -0.1620798300 \\
& 32 & -1.1810598785 & +0.2650033145 & +0.4128924378 & -0.1616366743 \\
& 64 & -1.1810598778 & +0.2650033154 & +0.4128924376 & -0.1616366728 \\
& 128 & -1.1810598778 & +0.2650033154 & +0.4128924376 & -0.1616366728 \\
\hline
\end{tabular}




\section{Kapitel 5}

\section{Das inverse Streuproblem}

In diesem Kapitel wird das inverse Streuproblem behandelt. Es ist die Gestalt des Streukörpers, unter Kenntnis der Wellenzahl $k$, zu ermitteln. Als Daten stehen Fernfelder zu gestreuten Feldern bei aus unterschiedlichen Richtungen einfallenden ebenen Wellen zur Verfügung. Im gesamten Kapitel werden nur zulässige Gebiete $D \subset \mathbb{R}^{2}$ im Sinne von Kapitel $⿴ 囗 ⿱ 一 一)$ betrachtet.

Im Abschnitt 5.1 wird das Problem der Eindeutigkeit untersucht, in den Abschnitten 5.2 und 5.3 die Fréchet-Differenzierbarkeit des Fernfeldoperators gezeigt sowie deren Ableitung durch ein Randwertproblem charakterisiert.

\subsection{Eindeutigkeit beim inversen Streuproblem}

Satz 5.1 Es seien $D_{1}$ und $D_{2}$ zwei Streugebiete derart, dass die Fernfelder der zugehörigen Streuprobleme bei fester Wellenzahl und allen Einfallsrichtungen einfallender ebener Wellen übereinstimmen, d.h.

$$
u_{\infty, 1}(\hat{x}, d)=u_{\infty, 2}(\hat{x}, d) \text { für alle } \hat{x}, d \in \Omega .
$$

Dann gilt $D_{1}=D_{2}$.

Beweis: Sei $G$ die unbeschränkte Komponente von $\mathbb{R}^{2} \backslash\left(\overline{D_{1}} \cup \overline{D_{2}}\right)$.

1. Aus der Übereinstimmung der Fernfelder folgt aus dem Rellich-Lemma die Übereinstimmung der gestreuten Felder, das heißt

$$
u_{1}^{s}(y, d)=u_{2}^{s}(y, d) \text { für alle } y \in G, d \in \Omega .
$$


2. Für festes $y \in G$ betrachte man die Streuprobleme (bezüglich der Gebiete $D_{1}$ und $D_{2}$ ) bei einfallenden Wellen

$$
w^{i}(x, y)=\Phi_{k}(x, y)=\frac{i}{4} H_{0}^{(1)}(k|x-y|) .
$$

Mit anderen Worten: Gesucht werden $w_{j}^{s} \in C^{2}\left(\mathbb{R}^{2} \backslash \overline{D_{j}}\right) \cap C\left(\mathbb{R}^{2} \backslash D_{j}\right)$ mit den Eigenschaften

$$
\begin{aligned}
\triangle w_{j}^{s}(\cdot, y)+k^{2} w_{j}^{s}(\cdot, y) & =0 \text { in } \mathbb{R}^{2} \backslash \overline{D_{j}}, \\
w_{j}^{s}(x, y) & =-\Phi_{k}(x, y) \text { für } x \in \partial D_{j}, \\
\lim _{r \rightarrow \infty} r^{1 / 2}\left(\frac{\partial w_{j}^{s}(\cdot, y)}{\partial r}-i k w_{j}^{s}(\cdot, y)\right) & =0, r=|x|,
\end{aligned}
$$

gleichmäßig für alle Richtungen $\hat{x}=x /|x|$. Unter Ausnutzen der Symmetrie

$$
w_{j}^{s}(x, y)=w_{j}^{s}(y, x), x, y \in G, j=1,2,
$$

lässt sich mit Hilfe der Greenschen Sätze die gemischte Reziprozität

$$
u_{j}^{s}(y, d)=4 \pi w_{\infty, j}(-d, y) \text { für alle } y \in G, d \in \Omega,
$$

beweisen.

3. Aus den beiden ersten Teilen folgt unmittelbar

$$
w_{\infty, 1}(d, y)=w_{\infty, 2}(d, y) \text { für alle } y \in G, d \in \Omega .
$$

Das Rellich-Lemma liefert

$$
w_{1}^{s}(x, y)=w_{2}^{s}(x, y) \text { für alle } x, y \in G .
$$

4. Angenommen es gilt $D_{1} \neq D_{2}$. Dann existiert ohne Beschränkung der Allgemeinheit ein Punkt $y^{*} \in \partial G$ mit $y^{*} \in \partial D_{1}$ und $y^{*} \notin \overline{D_{2}}$. (Anmerkung: Der Fall $D_{1} \cap D_{2}=\emptyset$ kann ausgeschlossen werden, denn ganze Lösungen der Helmholtzgleichung, die die Sommerfeldsche Ausstrahlungsbedingung erfüllen, verschwinden auf ganz $\mathbb{R}^{2}$.) 
Weiterhin existiert ein $h>0$ so, dass zum einen für die abgeschlossene Kugel $B:=$ $B\left[y^{*}, h\right]=\left\{y \in \mathbb{R}^{2}|| y-y^{*} \mid \leq h\right\}$ die Beziehung $B \cap \overline{D_{2}}=\emptyset$ gilt und zum anderen die Folge

$$
y_{n}:=y^{*}+\frac{h}{n} \nu\left(y^{*}\right), n \in \mathbb{N}
$$

in $G$ enthalten ist.

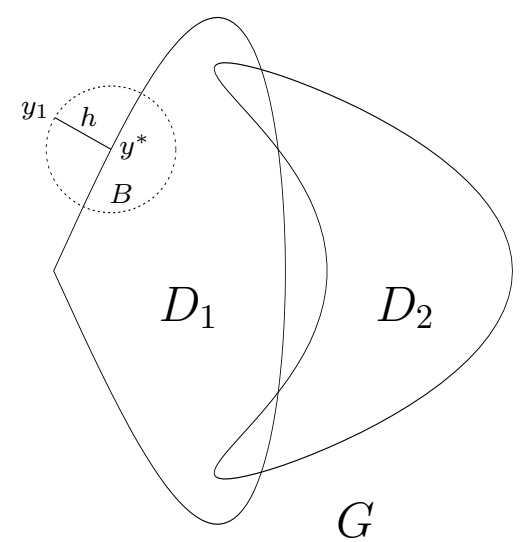

Einerseits existiert aufgrund der Wahl von $y^{*} \in G \backslash \overline{D_{2}}$ und der Wohlgestelltheit des Streuproblems bezüglich $D_{2}$ der Grenzwert

$$
\lim _{n \rightarrow \infty} w_{2}^{s}\left(y^{*}, y_{n}\right)=w_{2}^{s}\left(y^{*}, y^{*}\right)
$$

und andererseits ist wegen der Randbedingung auf $\partial D_{1}$

$$
\lim _{n \rightarrow \infty} w_{1}^{s}\left(y^{*}, y_{n}\right)=-\lim _{n \rightarrow \infty} w^{i}\left(y^{*}, y_{n}\right)=-\frac{i}{4} \lim _{n \rightarrow \infty} H_{0}^{(1)}\left(\frac{k h}{n}\right)=\infty .
$$

Die Resultate in (5.3) und (5.4) widersprechen (5.2).

\subsection{Ableitung der Randintegraloperatoren}

Sei $D$ ein zulässiges Gebiet mit nur einer Ecke. Die im Raum $C(\partial D)$ zu lösende Randintegralgleichung

$$
\begin{aligned}
2 f(x)= & \varphi(x)+2 \int_{\partial D}\left[\frac{\partial \Phi_{k}}{\partial \nu(y)}(x, y)-i \eta \Phi_{k}(x, y)\right]\left(\varphi(y)-\varphi\left(x_{0}\right)\right) d s(y) \\
& +\varphi\left(x_{0}\right) \gamma(x), \quad x \in \partial D,
\end{aligned}
$$

mit der auf $\partial D$ stetigen Funktion $\gamma$,

$$
\gamma(x)=2 \int_{\partial D}\left(\frac{\partial \Phi_{k}}{\partial \nu(y)}(x, y)-\frac{\partial \Phi_{0}}{\partial \nu(y)}(x, y)-i \eta \Phi_{k}(x, y)\right) d s(y)-1
$$


wurde im Abschnitt 4.6 bereits parametrisiert. Von der Randkurve $z \in C_{2 \pi}(\mathbb{R}, \partial D)$ werden nun nachfolgende (leicht verschärfte) Eigenschaften vorausgesetzt:

- $\left.z\right|_{[0,2 \pi]} \in C^{2, \alpha}\left([0,2 \pi], \mathbb{R}^{2}\right)$,

- $\left.z\right|_{[0,2 \pi)}$ ist injektiv und linksorientiert,

- $z(0)=x_{0},\left|z^{\prime}(t)\right|>0 \forall t \in \mathbb{R}$ und

- $z^{\prime}(0) \cdot z^{\prime}(2 \pi) \neq-\left|z^{\prime}(0)\right|\left|z^{\prime}(2 \pi)\right|$.

Schreibt man die resultierende Gleichung (4.21) in Operatorform, so erhält man

$$
\tilde{f}(t)=\left(I+K_{k}-i \eta S_{k}\right) \psi(t)+\psi(0) \tilde{\gamma}(t), 0 \leq t \leq 2 \pi,
$$

mit den Funktionen $\tilde{f}(t):=2 f(z(t)), \psi(t):=\varphi(z(t)), \tilde{\gamma}(t):=\gamma(z(t))$ und den Operatoren

$$
\begin{aligned}
S_{k}, K_{k} & : C_{2 \pi}(\mathbb{R}, \mathbb{C}) \longrightarrow C_{2 \pi}(\mathbb{R}, \mathbb{C}) \\
\left(S_{k} \psi\right)(t) & =\frac{i}{2} \int_{0}^{2 \pi} H_{0}^{(1)}(k|z(t)-z(\tau)|)\left|z^{\prime}(\tau)\right|[\psi(\tau)-\psi(0)] d \tau \\
\left(K_{k} \psi\right)(t) & =\frac{i k}{2} \int_{0}^{2 \pi} H_{1}^{(1)}(k|z(t)-z(\tau)|) \frac{z^{\prime}(\tau)^{\perp} \cdot[z(t)-z(\tau)]}{|z(t)-z(\tau)|}[\psi(\tau)-\psi(0)] d \tau .
\end{aligned}
$$

Die stetige Funktion $\tilde{\gamma}$ entspricht (im wesentlichen) dem Potenzialoperator $K_{k}-K_{0}-i \eta S_{k}$, bei dem anstatt $\psi(\tau)-\psi(0)$ die konstante Dichte 1 auftritt. Es gilt demnach

$$
\begin{aligned}
\tilde{\gamma}(t)= & \int_{0}^{2 \pi}\left(\frac{i k}{2} H_{1}^{(1)}(k|z(t)-z(\tau)|)+\frac{1}{\pi} \frac{1}{|z(t)-z(\tau)|)}\right) \frac{z^{\prime}(\tau)^{\perp} \cdot[z(t)-z(\tau)]}{|z(t)-z(\tau)|} d \tau \\
& +\frac{\eta}{2} \int_{0}^{2 \pi} H_{0}^{(1)}(k|z(t)-z(\tau)|)\left|z^{\prime}(\tau)\right| d \tau-1
\end{aligned}
$$

Wir werden der Einfachheit halber $\gamma$ und $f$ statt $\tilde{\gamma}$ und $\tilde{f}$ schreiben und $\gamma$ als Multiplikationsoperator $\gamma_{k}$ auffassen, sodass die Randintegralgleichung die Form

$$
f=\left(I+K_{k}-i \eta S_{k}+\gamma_{k}\right) \psi
$$

erhält.

Um die Abhängigkeit der Operatoren $S_{k}, K_{k}, \gamma_{k}$ von der Parametrisierung $z$ zu verdeutlichen, werden wir auch $S_{k}(z), K_{k}(z)$ und $\gamma_{k}(z)$ schreiben. Unter $S_{k}(\varphi, z)$ (bzw. $S_{k}(\varphi, z, t)$ ) 
verstehen wir die Funktion $S_{k}(z) \varphi$ (bzw. die komplexe Zahl $\left(S_{k}(z) \varphi\right)(t)$, also $S_{k}$ angewendet auf $\varphi$ und ausgewertet an der Stelle $t$ ).

Wir fassen die Menge der zulässigen Parametrisierungen

$$
\begin{aligned}
U:=\left\{z \in C_{2 \pi}\left(\mathbb{R}, \mathbb{R}^{2}\right) \mid\right. & \left.z\right|_{[0,2 \pi]} \in C^{2, \alpha}\left([0,2 \pi], \mathbb{R}^{2}\right),\left.z\right|_{[0,2 \pi)} \text { injektiv und linksorientiert, } \\
& \left.\left|z^{\prime}(t)\right|>0 \forall t \in \mathbb{R}, z^{\prime}(0) \cdot z^{\prime}(2 \pi) \neq-\left|z^{\prime}(0)\right|\left|z^{\prime}(2 \pi)\right|\right\}
\end{aligned}
$$

als Teilmenge des normierten Raumes

$$
X:=C_{2 \pi} \cap C^{2, \alpha}:=\left\{h \in C_{2 \pi}\left(\mathbb{R}, \mathbb{R}^{2}\right)|h|_{[0,2 \pi]} \in C^{2, \alpha}\left([0,2 \pi], \mathbb{R}^{2}\right)\right\}
$$

auf und verwenden als Norm

$$
\|h\|_{C^{2}[0,2 \pi]}:=\max \left\{\|h\|_{\infty},\left\|h^{\prime}\right\|_{\infty},\left\|h^{\prime \prime}\right\|_{\infty}\right\} .
$$

Auf $C_{2 \pi}(\mathbb{R}, \mathbb{C})$ wird die Norm $\|\varphi\|_{\infty, 0}=|\varphi(0)|+\max _{0 \leq t \leq 2 \pi}|\varphi(t)-\varphi(0)|$ verwendet.

Mit dem nachfolgenden Differenziationssatz wird die Fréchet-Differenzierbarkeit der Randintegraloperatoren gezeigt.

Satz 5.2 Sei $X$ ein normierter Raum, $U \subset X$ offen,

$$
Q=\left\{(t, \tau) \in[0,2 \pi]^{2} \mid t \neq \tau\right\} \backslash\{(0,0),(0,2 \pi),(2 \pi, 0),(2 \pi, 2 \pi)\},
$$

und $f=f_{1} \cdots f_{n}, f_{l}: Q \times U \longrightarrow \mathbb{C},(t, \tau, z) \mapsto f_{l}(t, \tau, z), l=1, \ldots, n$, ein Produkt von auf $Q \times U$ erklärten Funktionen, die (bei festem dritten Argument) bezüglich der beiden ersten Variablen $2 \pi$-periodisch sind und nachfolgende Eigenschaften besitzen.

1. Die Funktionen $f_{l}, l=1, \ldots, n$ sind für festes $(t, \tau) \in Q$ zweimal stetig Fréchetdifferenzierbar nach der dritten Variablen.

2. Für jedes $z \in U$ und $h \in X$ sind $f(\cdot, \cdot, z)$ und $\frac{\partial f}{\partial z}(\cdot, \cdot, z, h)$ auf $[0,2 \pi] \times[0,2 \pi]$ stetig oder schwach singulär oder bei den Ecken des Quaders $[0,2 \pi] \times[0,2 \pi]$ durch einen Mellin-Kern abschätzbar, d.h. $f(\cdot, \cdot, z)$ und $\frac{\partial f}{\partial z}(\cdot, \cdot, z, h)$ sind stetig auf $[0,2 \pi] \times$ $[0,2 \pi] \backslash\{(0,0),(0,2 \pi),(2 \pi, 0),(2 \pi, 2 \pi)\}$ und es gebe (von $z$ und $h$ abhängige) stetig differenzierbare Funktionen $k_{1}, \ldots, k_{8}:[0, \infty) \rightarrow[0, \infty)$ und $T>0$ hinreichend 
klein, sodass gilt $k_{l}(0)=0, \int_{0}^{\infty} \frac{k_{l}(s)}{s} d s<\infty, l=1, \ldots, 8$,

$$
\begin{aligned}
|f(t, \tau, z)| & \leq \frac{1}{\tau} k_{1}\left(\frac{t}{\tau}\right), & & 0 \leq t \leq T, 0<\tau \leq T, \\
|f(t, 2 \pi-\tau, z)| & \leq \frac{1}{\tau} k_{2}\left(\frac{t}{\tau}\right), & & 0 \leq t \leq T, 0<\tau \leq T, \\
|f(2 \pi-t, \tau, z)| & \leq \frac{1}{\tau} k_{3}\left(\frac{t}{\tau}\right), & & 0 \leq t \leq T, 0<\tau \leq T, \\
|f(2 \pi-t, 2 \pi-\tau, z)| & \leq \frac{1}{\tau} k_{4}\left(\frac{t}{\tau}\right), & & 0 \leq t \leq T, 0<\tau \leq T,
\end{aligned}
$$

und desgleichen für $\frac{\partial f}{\partial z}(\cdot, \cdot, z, h)$ mit Kernen $k_{5}, \cdots, k_{8}$.

3. Für ein $z_{0} \in U$ existieren integrierbare Funktionen $g_{l}^{(j)}: Q \rightarrow[0, \infty), j=0,1,2$, $l=1, \ldots, n$ und Konstanten $R, C>0$ so, dass

$$
\begin{gathered}
\left|\frac{\partial^{j} f_{l}}{\partial z^{j}}(t, \tau, z, h)\right| \leq g_{l}^{(j)}(t, \tau)\|h\|_{X}^{j} \text { für }(t, \tau) \in Q, z \in B\left[z_{0}, R\right] \subset U, h \in X, j=0,1,2, \\
\sup _{0<t<2 \pi} \int_{0}^{2 \pi} g_{1}^{\left(j_{1}\right)} \ldots g_{n}^{\left(j_{n}\right)}(t, \tau) d \tau \leq C \quad \begin{array}{l}
\text { für alle } j=\left(j_{1}, \ldots, j_{n}\right) \in \mathbb{N}_{0}^{n} \text { mit } \\
|j|:=j_{1}+\ldots+j_{n} \leq 2 .
\end{array}
\end{gathered}
$$

Dann sind die Abbildungen

$$
\begin{aligned}
A(z) & : \quad C_{2 \pi}(\mathbb{R}, \mathbb{C}) \rightarrow C_{2 \pi}(\mathbb{R}, \mathbb{C}),(A(z) \varphi)(t)=\int_{0}^{2 \pi} f(t, \tau, z)[\varphi(\tau)-\varphi(0)] d \tau, \\
A^{1}(z, h): & C_{2 \pi}(\mathbb{R}, \mathbb{C}) \rightarrow C_{2 \pi}(\mathbb{R}, \mathbb{C}),\left(A^{1}(z, h) \varphi\right)(t)=\int_{0}^{2 \pi} \frac{\partial f}{\partial z}(t, \tau, z, h)[\varphi(\tau)-\varphi(0)] d \tau
\end{aligned}
$$

für alle $z \in U$ und $h \in X$ wohldefiniert, linear und beschränkt. Desweiteren ist die Abbildung

$$
A: U \longrightarrow \mathcal{L}\left(C_{2 \pi}, C_{2 \pi}\right), \quad z \mapsto A(z)
$$

Fréchet-differenzierbar in $z_{0}$ mit $A^{\prime}\left(z_{0}, h\right)=A^{1}\left(z_{0}, h\right)$.

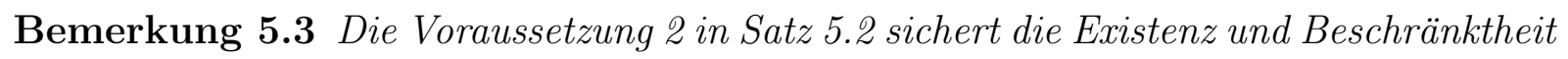
von $A(z)$ und $A^{1}(z, h)$. Aufgrund der Linearität des Integrals und der Fréchet-Ableitung darf die Forderung 2. an $f$ auch modifiziert werden: $f(\cdot, \cdot, z)$ ist Summe aus einem stetigen, einem schwach singulären und einem in den Ecken singulären Anteil, der sich wie ein Mellin-Kern verhält. 
Bemerkung 5.4 Die Forderung 2., nach dem $f(\cdot, \cdot, z)$ auf $[0,2 \pi] \times[0,2 \pi]$ stetig ist, kann abgeschwächt werden zu beschränkt auf $[0,2 \pi] \times[0,2 \pi]$ und bis auf die Ecken stetig.

Bemerkung 5.5 Läßt man in Satz 5.9 nur stetige oder schwach singuläre Kerne zu und ersetzt für ein Gebiet $G \subset \mathbb{R}^{2}$ die Menge $Q$ durch $G \times[0,2 \pi]$, so bleibt die Aussage des Satzes ebenfalls richtig.

Beweis: Vergleiche [47, Theorem 1] oder [51, Satz 4.8].

Beweis von Satz 5.2: 1. Zunächst wird die Abbildungseigenschaft

$$
A(z), A^{1}(z, h) \in \mathcal{L}\left(C_{2 \pi}, C_{2 \pi}\right)
$$

diskutiert. Seien dazu $z \in U$ und $h \in X$ beliebig, aber fest. Für auf $[0,2 \pi] \times[0,2 \pi]$ stetige oder schwach singuläre Kerne $f(\cdot, \cdot, z)$ bzw. $\frac{\partial f}{\partial z}(\cdot, \cdot, z, h)$ sind die Operatoren $A(z)$ und $A^{1}(z, h)$ nach Satz 2.7 (Abschnitt 2.3) aus $\mathcal{L}\left(C_{2 \pi}(\mathbb{R}, \mathbb{C}), C_{2 \pi}(\mathbb{R}, \mathbb{C})\right)$. In allen anderen Fällen sind nach Voraussetzung 2. $f(\cdot, \cdot, z)$ und $\frac{\partial f}{\partial z}(\cdot, \cdot, z, h)$ bis auf die Ecken stetig und verhalten sich in den Ecken wie ein Mellin-Kern. Mit einer geeigneten Abschneidefunktion lassen sich diese Kerne aufspalten in einen stetigen Anteil und vier in jeweils einer Ecke singuläre Anteile, deren Träger jeweils in einer $T$-Umgebung der Ecke des Quadrats $[0,2 \pi] \times[0,2 \pi]$ liegt. Die Abbildungseigenschaft von $A$ und die Existenz von $\|A\|_{\infty, 0}$ folgen dann aus [28, Theorem 3.1]. Die gleiche Argumentation für $\frac{\partial f}{\partial z}$ führt zu $A^{1}(z, h) \in \mathcal{L}\left(C_{2 \pi}, C_{2 \pi}\right)$.

2. Als nächstes ist nachzuweisen, dass die Abbildung

$$
X \rightarrow \mathcal{L}\left(C_{2 \pi}, C_{2 \pi}\right), \quad h \mapsto A^{1}\left(z_{0}, h\right)
$$

linear und beschränkt ist. Nach der ersten Voraussetzung ist $h \mapsto \frac{\partial f}{\partial z}(t, \tau, z, h)$ für festes $z \in U$ und alle $(t, \tau) \in Q$ linear. Demnach gilt für $z \in U, \varphi \in C_{2 \pi}$ und $0<t<2 \pi$

$$
\left(A^{1}\left(z, \lambda h_{1}+\mu h_{2}\right) \varphi\right)(t)=\lambda\left(A^{1}\left(z, h_{1}\right) \varphi\right)(t)+\mu\left(A^{1}\left(z, h_{2}\right) \varphi\right)(t) .
$$

Aus Stetigkeitsgründen ist obige Gleichheit auch gültig für $t=0$. Damit ist $h \mapsto A^{1}(z, h)$ für jedes $z \in U$ linear. Mit Voraussetzung [3. für die erste Fréchet-Ableitung von $f$ wird abgeschätzt

$$
\left|\left(A^{1}\left(z_{0}, h\right) \varphi\right)(t)\right| \leq \int_{0}^{2 \pi}\left|\frac{\partial f}{\partial z}\left(t, \tau, z_{0}, h\right)\right||\varphi(\tau)-\varphi(0)| d \tau
$$




$$
\begin{aligned}
& \leq \int_{0}^{2 \pi} \sum_{j \in \mathbb{N}_{0}^{n},|j|=1}\left(g_{1}^{\left(j_{1}\right)} \cdots g_{n}^{\left(j_{n}\right)}\right)(t, \tau)\|h\|_{X}|\varphi(\tau)-\varphi(0)| d \tau \\
& \leq 2 \pi n C\|\varphi\|_{\infty, 0}\|h\|_{X} \text { für alle } t \in \mathbb{R}, \varphi \in C_{2 \pi} .
\end{aligned}
$$

Damit folgt $\left\|A^{1}\left(z_{0}, h\right) \varphi\right\|_{\infty, 0} \leq \tilde{C}\|\varphi\|_{\infty, 0}\|h\|_{X}$ für jedes $\varphi \in C_{2 \pi}$ und daher

$$
\left\|A^{1}\left(z_{0}, h\right)\right\|_{\mathcal{L}\left(C_{2 \pi}, C_{2 \pi}\right)} \leq \tilde{C}\|h\|_{X} \text { für alle } h \in X .
$$

3. Es bleibt $\left\|A\left(z_{0}+h\right)-A\left(z_{0}\right)-A^{1}\left(z_{0}, h\right)\right\|=o\left(\|h\|_{X}\right)$ nachzurechnen. Für $(t, \tau) \in Q$ und $\|h\|_{X}<R$ gilt

$$
\begin{aligned}
& \left|f\left(t, \tau, z_{0}+h\right)-f\left(t, \tau, z_{0}\right)-\frac{\partial f}{\partial z}\left(t, \tau, z_{0}, h\right)\right| \\
& \quad \leq \int_{0}^{1}|1-\vartheta|\left|\frac{\partial^{2} f}{\partial z^{2}}\left(t, \tau, z_{0}+\vartheta h, h\right)\right| d \vartheta \\
& \quad \leq \sum_{|j|=2}\left(g_{1}^{\left(j_{1}\right)} \ldots g_{n}^{\left(j_{n}\right)}\right)(t, \tau)\|h\|_{X}^{2}
\end{aligned}
$$

und wegen der Normäquivalenz $\|\varphi\|_{\infty, 0} \leq 3\|\varphi\|_{\infty} \leq 3\|\varphi\|_{\infty, 0}$ sowie

$$
\begin{aligned}
& \left\|A\left(z_{0}+h\right)-A\left(z_{0}\right)-A^{1}\left(z_{0}, h\right)\right\|_{\mathcal{L}\left(C_{2 \pi}, C_{2 \pi}\right)} \\
& =\sup _{\|\varphi\|_{\infty, 0}=1}\left\|\int_{0}^{2 \pi}\left[f\left(\cdot, \tau, z_{0}+h\right)-f\left(\cdot, \tau, z_{0}\right)-\frac{\partial f}{\partial z}\left(\cdot, \tau, z_{0}, h\right)\right](\varphi(\tau)-\varphi(0)) d \tau\right\|_{\infty, 0} \\
& \leq 3\|h\|_{X}^{2} \sum_{|j|=2} \sup _{0<t<2 \pi} \int_{0}^{2 \pi} g_{1}^{\left(j_{1}\right)} \ldots g_{n}^{\left(j_{n}\right)}(t, \tau) d \tau \\
& \leq C\|h\|_{X}^{2}
\end{aligned}
$$

ergibt sich die Behauptung.

Lemma 5.6 Die Menge der zulässigen Parametrisierungen $U$ ist offen im Raum $X=\left\{h \in C_{2 \pi}\left(\mathbb{R}, \mathbb{R}^{2}\right)|h|_{[0,2 \pi]} \in C^{2, \alpha}\left([0,2 \pi], \mathbb{R}^{2}\right)\right\}$

Beweis: $\frac{z^{\prime}(0) \cdot z^{\prime}(2 \pi)}{\left|z^{\prime}(0)\right|\left|z^{\prime}(2 \pi)\right|}$ ist stetig in $z$. Daher folgt die Behauptung aus [2], Lemma 3.7]).

Für die Anwendung von Satz 5.2 auf das modifizierte Einfach- und Doppelschichtpotenzial, werden vorab einige Lemmata bewiesen. Das nächste Lemma zeigt für eine Klasse von Kernen $f(t, \tau, z)$ die zweimalige stetige Fréchet-Differenzierbarkeit nach der dritten Variablen. 
Lemma 5.7 Für $(t, \tau) \in Q=\left\{(x, y) \in[0,2 \pi]^{2} \mid x \neq y\right\} \backslash\{(0,0),(2 \pi, 0),(0,2 \pi),(2 \pi, 2 \pi)\}$, $z \in U$ und $h \in X$, sind die nachfolgenden Abbildungen zweimal Fréchet-differenzierbar von $U$ nach $\mathbb{R}$ bzw. $\mathbb{R}^{2}$

1. $F_{1}: z \rightarrow z(t)-z(\tau)$ mit $F_{1}^{\prime}(z, h)=h(t)-h(\tau)$ und $F_{1}^{\prime \prime}(z, h, \tilde{h})=(0,0) \in \mathbb{R}^{2}$,

2. $F_{2}: z \rightarrow|z(t)-z(\tau)|$ mit $F_{2}^{\prime}(z, h)=\frac{[z(t)-z(\tau)] \cdot[h(t)-h(\tau)]}{|z(t)-z(\tau)|}$ und

$$
F_{2}^{\prime \prime}(z, h)=\frac{|h(t)-h(\tau)|^{2}}{|z(t)-z(\tau)|}-\frac{|[z(t)-z(\tau)] \cdot[h(t)-h(\tau)]|^{2}}{|z(t)-z(\tau)|^{3}},
$$

3. $F_{3}: z \rightarrow z^{\prime}(t)$ mit $F_{3}^{\prime}(z, h)=h^{\prime}(t)$ und $F_{3}^{\prime \prime}(z, h, \tilde{h})=(0,0) \in \mathbb{R}^{2}$,

4. $F_{4}: z \rightarrow\left|z^{\prime}(t)\right|$ mit $F_{4}^{\prime}(z, h)=\frac{z^{\prime}(t) \cdot h^{\prime}(t)}{\left|z^{\prime}(t)\right|}$ und

$$
F_{4}^{\prime \prime}(z, h)=\frac{\left|h^{\prime}(t)\right|^{2}}{\left|z^{\prime}(t)\right|}-\frac{\left|z^{\prime}(t) \cdot h^{\prime}(t)\right|^{2}}{\left|z^{\prime}(t)\right|^{3}} .
$$

Beweis: Anwenden der Rechenregeln zur Fréchet-Ableitung.

Lemma 5.8 Seien $z \in U$ eine zulässige Parametrisierung und $(t, \tau) \in Q$. Dann sind die Kerne der Integraloperatoren $S_{k}(z), K_{k}(z)$ und $\gamma_{k}(z)$ zweimal stetig Fréchet-differenzierbar.

Beweis: Für $z \in U$ und $0<t, \tau<2 \pi, t \neq \tau$ erklären wir die Kerne

$$
\begin{aligned}
M_{k}(t, \tau, z) & :=\frac{i}{2} H_{0}(k|z(t)-z(\tau)|)\left|z^{\prime}(\tau)\right|, \\
L_{0}(t, \tau, z) & :=\frac{1}{\pi} \frac{1}{|z(t)-z(\tau)|^{2}}, \\
L_{k}(t, \tau, z) & :=-\frac{i k}{2} \frac{H_{0}^{\prime}(k|z(t)-z(\tau)|)}{|z(t)-z(\tau)|}, \\
q(t, \tau, z) & :=\left(z_{2}^{\prime}(\tau),-z_{1}^{\prime}(\tau)\right) \cdot[z(t)-z(\tau)] .
\end{aligned}
$$


Dann lassen sich die Operatoren $S_{k}(z), K_{k}(z)$ und $\gamma_{k}(z)$ in nachfolgender Form darstellen

$$
\begin{aligned}
\left(S_{k}(z) \psi\right)(t) & =\int_{0}^{2 \pi} M_{k}(t, \tau, z)[\psi(\tau)-\psi(0)] d \tau \\
\left(K_{k}(z) \psi\right)(t) & =\int_{0}^{2 \pi} L_{k}(t, \tau, z) q(t, \tau, z)[\psi(\tau)-\psi(0)] d \tau \\
\left(\gamma_{k}(z) \psi\right)(t) & =\left(\int_{0}^{2 \pi}\left(\left(L_{k}-L_{0}\right) q-i \eta M_{k}\right)(t, \tau, z) d \tau-1\right) \psi(0) .
\end{aligned}
$$

Nach Lemma 5.7 sind die Kerne $M_{k}, L_{0}, L_{k}$ und $q$ für $(t, \tau) \in Q$ zweimal stetig differenzierbar nach der dritten Variablen. Die ersten Ableitungen lauten

$$
\begin{aligned}
M_{k}^{\prime}(t, \tau, z, h)= & \frac{i k}{2} H_{0}^{\prime}(k|z(t)-z(\tau)|) \frac{[z(t)-z(\tau)] \cdot[h(t)-h(\tau)]}{|z(t)-z(\tau)|}\left|z^{\prime}(\tau)\right| \\
& +\frac{i}{2} H_{0}(k|z(t)-z(\tau)|) \frac{z^{\prime}(\tau) \cdot h^{\prime}(\tau)}{\left|z^{\prime}(\tau)\right|} \\
L_{0}^{\prime}(t, \tau, z, h)= & \frac{-2}{\pi} \frac{[z(t)-z(\tau)] \cdot[h(t)-h(\tau)]}{|z(t)-z(\tau)|^{4}} \\
L_{k}^{\prime}(t, \tau, z, h)= & -\frac{i k}{2}\left\{\frac{k H_{0}^{\prime \prime}(k|z(t)-z(\tau)|)}{|z(t)-z(\tau)|}-\frac{H_{0}^{\prime}(k|z(t)-z(\tau)|)}{|z(t)-z(\tau)|^{2}}\right\} \times \\
& \times \frac{[z(t)-z(\tau)] \cdot[h(t)-h(\tau)]}{|z(t)-z(\tau)|} \\
q^{\prime}(t, \tau, z, h)= & \left(h_{2}^{\prime}(\tau),-h_{1}^{\prime}(\tau)\right) \cdot[z(t)-z(\tau)]+\left(z_{2}^{\prime}(\tau),-z_{1}^{\prime}(\tau)\right) \cdot[h(t)-h(\tau)] .
\end{aligned}
$$

Ziel ist nachzuweisen, dass die Fréchet-Ableitungen von $z \mapsto S_{k}(z), z \mapsto K_{k}(z)$ und $z \mapsto \gamma_{k}(z)$ gegeben sind durch

$$
\begin{array}{rlrl}
S_{k}^{\prime}(z) & : & C_{2 \pi}(\mathbb{R}, \mathbb{C}) \cap C^{2}[0,2 \pi] \rightarrow \mathcal{L}\left(C_{2 \pi}, C_{2 \pi}\right) \\
& h \longmapsto S_{k}^{\prime}(\cdot, z, h) \\
\left(S_{k}^{\prime}(\varphi, z, h)\right)(t)= & \int_{0}^{2 \pi} M_{k}^{\prime}(t, \tau, z, h)[\varphi(\tau)-\varphi(0)] d \tau \\
K_{k}^{\prime}(z): & C_{2 \pi}(\mathbb{R}, \mathbb{C}) \cap C^{2}[0,2 \pi] \rightarrow \mathcal{L}\left(C_{2 \pi}, C_{2 \pi}\right) \\
& & h \longmapsto K_{k}^{\prime}(\cdot, z, h) \\
\left(K_{k}^{\prime}(\varphi, z, h)\right)(t):= & \int_{0}^{2 \pi}\left\{L_{k}^{\prime}(t, \tau, z, h) q(t, \tau, z)+L_{k}(t, \tau, z) q^{\prime}(t, \tau, z, h)\right\}[\varphi(\tau)-\varphi(0)] d \tau,
\end{array}
$$




$$
\begin{aligned}
& \gamma_{k}^{\prime}(z): C_{2 \pi}(\mathbb{R}, \mathbb{C}) \cap C^{2}[0,2 \pi] \rightarrow \mathcal{L}\left(C_{2 \pi}, C_{2 \pi}\right) \\
& h \longmapsto \gamma_{k}^{\prime}(\cdot, z, h) \\
&\left(\gamma_{k}^{\prime}(\varphi, z, h)\right)(t)=\quad \varphi(0) \int_{0}^{2 \pi}\left\{\left(L_{k}^{\prime}-L_{0}^{\prime}\right)(t, \tau, z, h) q(t, \tau, z)+\left(L_{k}-L_{0}\right)(t, \tau, z) q^{\prime}(t, \tau, z, h)\right. \\
&\left.\quad-i \eta M_{k}^{\prime}(t, \tau, z, h)\right\} d \tau .
\end{aligned}
$$

Für den Beweis sind die übrigen Voraussetzungen des Differenziationsatzes 5.2 einschließlich Bemerkung 5.3 zu prüfen.

Wegen $H_{n}^{(1)}(r)=J_{n}(r)+i Y_{n}(r)$ und der Darstellung (2.2) gilt für $H_{0}^{(1)}$

$$
\begin{aligned}
H_{0}^{(1)}(k|z(t)-z(\tau)|)= & \frac{i}{\pi} J_{0}(k|z(t)-z(\tau)|) \ln \left(4 \sin ^{2} \frac{t-\tau}{2}\right) \\
& +J_{0}(k|z(t)-z(\tau)|)\left[\frac{i}{\pi} \ln \left(\frac{|z(t)-z(\tau)|^{2}}{4 \sin ^{2} \frac{t-\tau}{2}}\right)+1+\frac{2 i}{\pi} \ln \frac{k}{2}+\frac{2 i}{\pi} C_{E}\right] \\
& -\frac{2 i}{\pi} \sum_{p=0}^{\infty} \frac{(-1)^{p}}{p ! p !}\left(\frac{k|z(t)-z(\tau)|}{2}\right)^{2 p} \psi(p)
\end{aligned}
$$

und wegen $(2.3)$ für $H_{1}^{(1)}$

$$
\begin{aligned}
H_{1}^{(1)}(k|z(t)-z(\tau)|)= & \frac{i}{\pi} J_{1}(k|z(t)-z(\tau)|) \ln \left(4 \sin ^{2} \frac{t-\tau}{2}\right) \\
& +J_{1}(k|z(t)-z(\tau)|)\left[\frac{i}{\pi} \ln \left(\frac{|z(t)-z(\tau)|^{2}}{4 \sin ^{2} \frac{t-\tau}{2}}\right)+1+\frac{2 i}{\pi} \ln \frac{k}{2}+\frac{2 i}{\pi} C_{E}\right] \\
& -\frac{i}{\pi} \sum_{p=0}^{\infty} \frac{(-1)^{p}}{p !(p+1) !}\left(\frac{k|z(t)-z(\tau)|}{2}\right)^{2 p+1}\{\psi(p+1)+\psi(p)\} \\
& -\frac{2 i}{\pi} \frac{1}{k|z(t)-z(\tau)|} .
\end{aligned}
$$

Satz 5.9 Sei $V: \mathbb{C} \rightarrow \mathbb{C}$ eine analytische und gerade Funktion und $z \in U$ eine zulässige Parametrisierung.

Für $(t, \tau) \in Q=\{(x, y) \in[0,2 \pi] \times[0,2 \pi] \mid x \neq y\} \backslash\{(0,0),(0,2 \pi),(2 \pi, 0),(2 \pi, 2 \pi)\}$ habe der Kern $f$ die Form

$$
f(t, \tau, z)=V(k|z(t)-z(\tau)|)\left|z^{\prime}(\tau)\right| \text { oder }
$$




$$
\begin{aligned}
& f(t, \tau, z)=V(k|z(t)-z(\tau)|)\left|z^{\prime}(\tau)\right| \ln \left(4 \sin ^{2} \frac{t-\tau}{2}\right) \quad \text { oder } \\
& f(t, \tau, z)=V(k|z(t)-z(\tau)|)\left|z^{\prime}(\tau)\right| \ln \left(\frac{|z(t)-z(\tau)|^{2}}{4 \sin ^{2} \frac{t-\tau}{2}}\right) .
\end{aligned}
$$

Dann erfüllt der Kern $f$ die Voraussetzungen aus Satz 5.9. Insbesondere ist die Abbildung $U \rightarrow \mathcal{L}\left(C_{2 \pi}, C_{2 \pi}\right), z \mapsto S_{k}(z)$, mit

$$
\left(S_{k}(z) \varphi\right)(t)=\int_{0}^{2 \pi} M_{k}(t, \tau, z)[\varphi(\tau)-\varphi(0)] d \tau, 0 \leq t \leq 2 \pi,
$$

Fréchet-differenzierbar mit Ableitung $X \rightarrow \mathcal{L}\left(C_{2 \pi}, C_{2 \pi}\right), h \mapsto S_{k}^{\prime}(z, h)$, wobei

$$
\left(S_{k}^{\prime}(z, h) \varphi\right)(t)=\int_{0}^{2 \pi} M_{k}^{\prime}(t, \tau, z, h)[\varphi(\tau)-\varphi(0)] d \tau, 0 \leq t \leq 2 \pi .
$$

Dabei sind die Kerne $M_{k}$ und $M_{k}^{\prime}$ durch (5.9) und (5.11) gegeben.

Beweis: Es werden die Voraussetzungen von Satz 5.2 geprüft.

1. Voraussetzung 1 ist wegen der Regularität von $V$ und Lemma 5.7 gegeben.

2. Als nächstes wird Voraussetzung 2 nachgerechnet. Die Funktionen $f$ sind in den beiden ersten Argumenten 2 $\pi$-periodisch. Im ersten Fall ist $f$ (bei festem $z \in U$ ) stetig auf $[0,2 \pi]^{2}$, im zweiten Fall schwach singulär. Es bleibt der Nachweis der Beschränktheit (siehe Bemerkung 5.3) von

$$
\ln \left(\frac{|z(t)-z(\tau)|^{2}}{4 \sin ^{2} \frac{t-\tau}{2}}\right)
$$

zu beweisen. Die Stetigkeit auf $Q \backslash\{(0,0),(2 \pi, 2 \pi),(0,2 \pi),(2 \pi, 0)\}$ ist offensichtlich. Eine Taylorentwicklung zeigt, dass der Ausdruck durch

$$
\ln \left(\left|z^{\prime}(t)\right|^{2}\right), 0 \leq t=\tau \leq 2 \pi
$$

stetig ergänzt wird. Es verbleibt die Beschränktheit bei $(0,2 \pi)$ und $(2 \pi, 0)$ nachzuweisen. Sei dazu $\delta>0$ hinreichend klein und

$$
0<\tau \leq \delta, 2 \pi-\delta \leq t<2 \pi
$$

Offenbar gilt

$$
\frac{|z(t)-z(\tau)|^{2}}{4 \sin ^{2} \frac{t-\tau}{2}}=\frac{|z(t)-z(\tau)|^{2} /(t-2 \pi-\tau)^{2}}{4 \sin ^{2}\left(\frac{t-\tau}{2}\right) /(t-2 \pi-\tau)^{2}}
$$


Der Nenner ist im Quadrat $[2 \pi-\delta, 2 \pi) \times(0, \delta]$ stetig, läßt sich durch 1 in $(2 \pi, 0)$ stetig ergänzen und hat keine Nullstellen. Der Zähler bleibt in obigem Quadrat beschränkt, denn aus dem Mittelwertsatz folgt

$$
|z(t)-z(\tau)|=|z(t-2 \pi)-z(\tau)| \leq \mid t-2 \pi-\tau) \mid\left\|z^{\prime}\right\|_{\infty}
$$

und damit

$$
\left|\frac{|z(t)-z(\tau)|^{2}}{(t-2 \pi-\tau)^{2}}\right| \leq\left\|z^{\prime}\right\|_{\infty}^{2},(t, \tau) \in[2 \pi-\delta, 2 \pi) \times(0, \delta] .
$$

Als nächstes ist die Voraussetzung 2 für die erste Ableitung $\frac{\partial f}{\partial z}$ zu prüfen. Mit der Bezeichnung $f_{1}(t, \tau, z)=V(k|z(t)-z(\tau)|)\left|z^{\prime}(\tau)\right|$ folgen aus der Produktregel (Satz 2.3) und Lemma 5.7 die Gleichungen

$$
\begin{aligned}
\frac{\partial f_{1}}{\partial z}(t, \tau, z, h)= & k V^{\prime}(k|z(t)-z(\tau)|) \frac{(z(t)-z(\tau)) \cdot(h(t)-h(\tau))}{|z(t)-z(\tau)|}\left|z^{\prime}(\tau)\right| \\
& +V(k|z(t)-z(\tau)|) \frac{z^{\prime}(\tau) \cdot h^{\prime}(\tau)}{\left|z^{\prime}(\tau)\right|} \text { oder } \\
\frac{\partial f}{\partial z}(t, \tau, z, h)= & \frac{\partial f_{1}}{\partial z}(t, \tau, z, h) \ln \left(4 \sin ^{2} \frac{t-\tau}{2}\right) \text { oder } \\
\frac{\partial f}{\partial z}(t, \tau, z, h)= & \frac{\partial f_{1}}{\partial z}(t, \tau, z, h) \ln \left(\frac{|z(t)-z(\tau)|^{2}}{4 \sin ^{2} \frac{t-\tau}{2}}\right) \\
& +f_{1}(t, \tau, z) 2 \frac{(z(t)-z(\tau)) \cdot(h(t)-h(\tau))}{|z(t)-z(\tau)|^{2}} .
\end{aligned}
$$

Offenbar ist $\frac{\partial f_{1}}{\partial z}$ stetig auf $[0,2 \pi]^{2}$ mit Ausnahme der Punkte $(0,2 \pi)$ und $(2 \pi, 0)$. Mit der Cauchy-Schwarzschen Ungleichung ergibt sich

$$
\left|\frac{(z(t)-z(\tau)) \cdot(h(t)-h(\tau))}{|z(t)-z(\tau)|}\right| \leq 2\|h\|_{\infty},
$$

und daher (bei festen $z$ und $h$ ) die Beschränktheit von $\frac{\partial f_{1}}{\partial z}(t, \tau, z, h)$ auf $Q$. Für $\frac{\partial f}{\partial z}$ wie in (5.14) ist der Ausdruck ebenfalls beschränkt, für $\frac{\partial f}{\partial z}$ wie in (5.13) ist der Term Summe aus einem schwach singulären und einem beschränkten Anteil.

3. Es bleibt das Prüfen der Voraussetzung 3. Dazu sei $z_{0} \in U$ beliebig, aber fest vorgegeben. Zu $z_{0}$ setze $R:=\frac{1}{2} \inf _{0 \leq \tau \leq 2 \pi}\left|z_{0}^{\prime}(\tau)\right|>0$. Für den Rest des Beweises seien $z \in$ $B\left[z_{0}, R\right] \subset U$ und $h \in X$. Der Radius $R$ wurde gerade so gewählt, dass $\inf _{0 \leq \tau \leq 2 \pi}\left|z^{\prime}(\tau)\right| \geq R$ für alle $z \in B\left[z_{0}, R\right]$ gilt. Der Integrand $f(\cdot, \cdot, z)$ ist jeweils ein Produkt der Terme 
$\left|z^{\prime}(\tau)\right|, V(k|z(t)-z(\tau)|)$ und $\ln \left(\frac{|z(t)-z(\tau)|^{2}}{4 \sin ^{2} \frac{t-\tau}{2}}\right)$. Sei zunächst $f(t, \tau, z)=\left|z^{\prime}(\tau)\right|$. Offenbar gilt

$$
\begin{aligned}
\frac{\partial f}{\partial z}(t, \tau, z, h) & =\frac{z^{\prime}(\tau) \cdot h^{\prime}(\tau)}{\left|z^{\prime}(\tau)\right|} \\
\frac{\partial^{2} f}{\partial z^{2}}(t, \tau, z, h) & =\frac{\left|h^{\prime}(\tau)\right|^{2}}{\left|z^{\prime}(\tau)\right|}-\frac{\left|z^{\prime}(\tau) \cdot h^{\prime}(\tau)\right|^{2}}{\left|z^{\prime}(\tau)\right|^{3}} .
\end{aligned}
$$

Mit der Cauchy-Schwarz-Ungleichung ergeben sich die Majoranten

$$
\begin{aligned}
|f(t, \tau, z)| & \leq R+\left\|z_{0}\right\|_{X} \leq C\left(R, z_{0}\right), \\
\left|\frac{\partial f}{\partial z}(t, \tau, z, h)\right| & \leq\left\|h^{\prime}\right\|_{\infty} \leq\|h\|_{X}, \\
\left|\frac{\partial^{2} f}{\partial z^{2}}(t, \tau, z, h)\right| & \leq 2 \frac{\left\|h^{\prime}\right\|_{\infty}^{2}}{\left|z^{\prime}(\tau)\right|} \leq \frac{2}{R}\|h\|_{X}^{2} .
\end{aligned}
$$

Für $f(t, \tau, z)=V(r)$, mit $r=k|z(t)-z(\tau)|$, ergeben sich

$$
\begin{aligned}
\frac{\partial f}{\partial z}(t, \tau, z, h)= & V^{\prime}(r) k \frac{(z(t)-z(\tau)) \cdot(h(t)-h(\tau))}{|z(t)-z(\tau)|} \\
\frac{\partial^{2} f}{\partial z^{2}}(t, \tau, z, h)= & V^{\prime \prime}(r) k^{2} \frac{[(z(t)-z(\tau)) \cdot(h(t)-h(\tau))]^{2}}{|z(t)-z(\tau)|^{2}} \\
& +V^{\prime}(r) k\left[\frac{|h(t)-h(\tau)|^{2}}{|z(t)-z(\tau)|}-\frac{|(z(t)-z(\tau)) \cdot(h(t)-h(\tau))|^{2}}{|z(t)-z(\tau)|^{3}}\right] .
\end{aligned}
$$

Da nach Voraussetzung an $V$ die Abbildungen $r \mapsto V(r), V^{\prime}(r), r^{-1} V^{\prime}(r), V^{\prime \prime}(r)$ auf der Menge $\left\{w \in \mathbb{C}|| w|\leq 2| k \mid\left(R+\left\|z_{0}\right\|_{X}\right)\right\}$ beschränkt sind, erhält man mit einer geeigneten Konstanten die Abschätzungen

$$
\left|\frac{\partial^{j} f}{\partial z^{j}}(t, \tau, z, h)\right| \leq C\left(z_{0}, R\right)\|h\|_{X}^{j}, j=0,1,2, z \in B\left[z_{0}, R\right] \subset U, h \in X .
$$

Schließlich sei $f(t, \tau, z)=\ln \left(\frac{|z(t)-z(\tau)|^{2}}{4 \sin ^{2} \frac{t-\tau}{2}}\right)$. Dann ergibt sich

$$
\begin{aligned}
\frac{\partial f}{\partial z}(t, \tau, z, h) & =2 \frac{(z(t)-z(\tau)) \cdot(h(t)-h(\tau))}{|z(t)-z(\tau)|^{2}} \\
\frac{\partial^{2} f}{\partial z^{2}}(t, \tau, z, h) & =2 \frac{|h(t)-h(\tau)|^{2}}{|z(t)-z(\tau)|^{2}}-4 \frac{|(z(t)-z(\tau)) \cdot(h(t)-h(\tau))|^{2}}{|z(t)-z(\tau)|^{4}} .
\end{aligned}
$$


Auch hier erhält man die Abschätzungen

$$
\begin{aligned}
|f(t, \tau, z, h)| & \leq C\left(z_{0}, R\right) \\
\left|\frac{\partial f}{\partial z}(t, \tau, z, h)\right| & \leq 2 \frac{\left\|h^{\prime}\right\|_{\infty}}{\inf _{0 \leq w \leq 2 \pi}\left|z^{\prime}(w)\right|} \leq C\left(z_{0}, R\right)\|h\|_{X}, \\
\left|\frac{\partial^{2} f}{\partial z^{2}}(t, \tau, z, h)\right| & \leq 6 \frac{\left\|h^{\prime}\right\|_{\infty}^{2}}{\inf _{0 \leq w \leq 2 \pi}\left|z^{\prime}(w)\right|^{2}} \leq C\left(z_{0}, R\right)\|h\|_{X}^{2} .
\end{aligned}
$$

Die Behauptung folgt nun aus dem Differenziationssatz 5.2, wobei die dortigen Funktionen $g_{l}^{(j)}$ in unserem Fall sämtlich konstante Funktionen sind.

Satz 5.10 Sei $W: \mathbb{C} \rightarrow \mathbb{C}$ eine analytische und ungerade Funktion sowie $z \in U$ eine zulässige Parametrisierung.

Für $(t, \tau) \in Q=\left\{(x, y) \in[0,2 \pi]^{2} \mid x \neq y\right\} \backslash\{(0,0),(2 \pi, 0),(0,2 \pi),(2 \pi, 2 \pi)\}$ habe der Kern $f$ die Form

$$
\begin{aligned}
f(t, \tau, z) & =\frac{W(k|z(t)-z(\tau)|)}{|z(t)-z(\tau)|}\left(z_{2}^{\prime}(\tau),-z_{1}^{\prime}(\tau)\right) \cdot[z(t)-z(\tau)] \ln \left(4 \sin ^{2} \frac{t-\tau}{2}\right) \text { oder } \\
f(t, \tau, z) & =\frac{W(k|z(t)-z(\tau)|)}{|z(t)-z(\tau)|}\left(z_{2}^{\prime}(\tau),-z_{1}^{\prime}(\tau)\right) \cdot[z(t)-z(\tau)] \ln \left(\frac{|z(t)-z(\tau)|^{2}}{4 \sin ^{2} \frac{t-\tau}{2}}\right) \text { oder } \\
f(t, \tau, z) & =\frac{1}{\pi} \frac{\left(z_{2}^{\prime}(\tau),-z_{1}^{\prime}(\tau)\right) \cdot[z(t)-z(\tau)]}{|z(t)-z(\tau)|^{2}} .
\end{aligned}
$$

Dann erfüllt der Kern $f$ die Voraussetzungen von Satz 5.9. Insbesondere ist die Abbildung $U \rightarrow \mathcal{L}\left(C_{2 \pi}, C_{2 \pi}\right), z \mapsto K_{k}(z)$, mit

$$
\left(K_{k}(z) \varphi\right)(t)=\int_{0}^{2 \pi} L_{k}(t, \tau, z) q(t, \tau, z)[\varphi(\tau)-\varphi(0)] d \tau, 0 \leq t \leq 2 \pi,
$$

Fréchet-differenzierbar mit Ableitung $X \rightarrow \mathcal{L}\left(C_{2 \pi}, C_{2 \pi}\right), h \mapsto K_{k}^{\prime}(z, h)$, wobei

$$
\left(K_{k}^{\prime}(z, h) \varphi\right)(t)=\int_{0}^{2 \pi}\left\{L_{k}^{\prime}(t, \tau, z, h) q(t, \tau, z)+L_{k}(t, \tau, z) q^{\prime}(t, \tau, z, h)\right\}[\varphi(\tau)-\varphi(0)] d \tau .
$$

Dabei sind die Kerne $L_{k}, q, L_{k}^{\prime}$ und $q^{\prime}$ durch (5.9) und (5.11) gegeben.

Beweis: Es werden erneut die Voraussetzungen von Satz 5.2 geprüft.

1. Voraussetzung 1 ist wegen der Regularität von $W$ erfüllt. 
2. Da $W$ analytisch und ungerade ist, ist

$$
\frac{W(k|z(t)-z(\tau)|)}{|z(t)-z(\tau)|},(t, \tau) \in[0,2 \pi] \times[0,2 \pi],
$$

stetig. In den beiden ersten Fällen ist $f$ Summe aus einem beschränkten und einem schwach singulären Anteil. Es ist lediglich

$$
f(t, \tau, z)=\frac{1}{\pi} \frac{\left(z_{2}^{\prime}(\tau),-z_{1}^{\prime}(\tau)\right) \cdot[z(t)-z(\tau)]}{|z(t)-z(\tau)|^{2}}
$$

zu untersuchen. Für $(t, \tau) \in Q$ ist der Kern stetig. Eine Taylorentwicklung zeigt, dass sich $f$ auf die Diagonale $0 \leq t=\tau \leq 2 \pi$ mit den Werten $\frac{1}{2 \pi} \frac{\left(z_{2}^{\prime}(t),-z_{1}^{\prime}(t)\right) \cdot z^{\prime \prime}(t)}{\left|z^{\prime}(t)\right|^{2}}$ stetig ergänzen lässt. Es verbleibt eine Betrachtung von $f$ bei den Ecken $(2 \pi, 0)$ und $(0,2 \pi)$. Obwohl an dieser Stelle der Nachweis aureicht, dass sich zu festem $z$ eine (von $z$ abhängige) integrierbare Majorante finden läßt, wird zur Vorbereitung von Teil 3 gleich allgemeiner argumentiert. Dazu sei $z_{0} \in U$ beliebig, aber fest vorgegeben. Man darf ohne Einschränkung $z_{0}^{\prime}(0) \neq z_{0}^{\prime}(2 \pi)$ voraussetzen, denn andernfalls hat das von $z_{0}$ berandete Gebiet keine Ecke und $f$ ist stetig. Für beliebiges $z \in U$ seien die Zahlen $a(z)=\left|z^{\prime}(0)\right|>0, b(z)=\left|z^{\prime}(2 \pi)\right|>0$ und $\beta(z)$ als Innenwinkel bei der Ecke $z(0)=x_{0}(z)$ erklärt.

Weiter werden Funktionen $k_{z}:[0, \infty) \rightarrow[0, \infty)$, mit

$$
k_{z}(s)=\frac{1}{\pi} \frac{a(z) b(z) s|\sin \beta(z)|}{[b(z) s-a(z) \cos (\beta(z))]^{2}+(a(z))^{2} \sin ^{2}(\beta(z))}, s \geq 0,
$$

erklärt. Aus Stetigkeitsgründen gibt es zu $z_{0}$ ein $R>0$ derart, dass für alle $z \in B\left[z_{0}, R\right] \subset$ $U$ die Abschätzung $0 \leq k_{z}(s) \leq 2 k_{z_{0}}(s), s \geq 0$ gültig ist. Für $a=a\left(z_{0}\right), b=b\left(z_{0}\right), \beta=$ $\beta\left(z_{0}\right)$ und $k=k_{z_{0}}$ lässt sich die Existenz von $\int_{0}^{\infty} \frac{k(s)}{s} d s$ nachweisen, denn für $0<\beta<\pi$ gilt

$$
\begin{aligned}
\int_{0}^{\infty} \frac{k(s)}{s} d s & =\frac{1}{\pi} \int_{0}^{\infty} \frac{\frac{b}{a \sin \beta}}{\left(\frac{b s-a \cos \beta}{a \sin \beta}\right)^{2}+1} d s \\
& =\frac{1}{\pi} \int_{-\cot \beta}^{\infty} \frac{1}{t^{2}+1} d t=\frac{1}{\pi}\left[\frac{\pi}{2}-\arctan (-\cot \beta)\right] \\
& =1-\frac{\beta}{\pi} .
\end{aligned}
$$


Ohne Einschränkung sei $R>0$ so klein gewählt, dass $\sin (\beta(z))$ auf $B\left[z_{0}, R\right] \subset U$ das Vorzeichen nicht wechselt. Dann ist der durch Linearisierung von $z$ erhaltene „Hauptteil $\tilde{f}$ von $f^{\prime \prime}$, mit

$$
\begin{aligned}
\tilde{f}(t, \tau, z) & =\frac{1}{\pi} \frac{z^{\prime}(2 \pi) \cdot\left(z^{\prime}(0)\right)^{\perp}(t-2 \pi)}{\left|z^{\prime}(2 \pi)\right|^{2}(t-2 \pi)^{2}+\left|z^{\prime}(0)\right|^{2} \tau^{2}-2 \tau(t-2 \pi)\left|z^{\prime}(0)\right|\left|z^{\prime}(2 \pi)\right| \cos \beta(z)} \\
& =\frac{1}{\pi} \frac{a(z) b(z)(t-2 \pi) \sin \beta(z)}{[b(z)(t-2 \pi)-a(z) \tau \cos \beta(z)]^{2}+(a(z))^{2} \sin ^{2} \beta(z)}
\end{aligned}
$$

wohldefiniert. $\mathrm{Zu} z_{0}$ und $R$ existiert ein $\delta>0$ so, dass

$$
|f(t, \tau, z)| \leq 2|\tilde{f}(t, \tau, z)| \text { für alle }(t, \tau) \in[2 \pi-\delta, 2 \pi] \times(0, \delta], z \in B\left[z_{0}, R\right] . \text { (5.15) }
$$

Um die Existenz von $\delta$ einzusehen, wird

$$
\begin{aligned}
& z(t)=z(2 \pi)+z^{\prime}(2 \pi)(t-2 \pi)+O(t-2 \pi)^{2}, \\
& z(\tau)=z(0)+z^{\prime}(0) \tau+O\left(\tau^{2}\right)
\end{aligned}
$$

entwickelt und damit folgt

$$
|z(t)-z(\tau)|^{2}=\left|z^{\prime}(2 \pi)\right|(t-2 \pi)^{2}+\left|z^{\prime}(0)\right|^{2} \tau^{2}-2(t-2 \pi) \tau z^{\prime}(2 \pi) \cdot z^{\prime}(0)+R_{2}(t, \tau, z)
$$

mit einem Restterm $R_{2}$. Wegen $z^{\prime}(\tau)=z^{\prime}(0)+O(\tau)$ ergibt sich

$$
\begin{aligned}
& f(t, \tau, z) \\
= & \frac{1}{\pi} \frac{z_{2}^{\prime}(0)\left[z_{1}^{\prime}(2 \pi)(t-2 \pi)-z_{1}^{\prime}(0) \tau\right]-z_{1}^{\prime}(0)\left[z_{2}^{\prime}(2 \pi)(t-2 \pi)-z_{2}^{\prime}(0) \tau\right]+R_{1}(t, \tau, z)}{\left|z^{\prime}(2 \pi)\right|^{2}(t-2 \pi)^{2}+\left|z^{\prime}(0)\right|^{2} \tau^{2}-2 \tau(t-2 \pi) z^{\prime}(0) \cdot z^{\prime}(2 \pi)+R_{2}(t, \tau, z)} \\
= & \frac{1}{\pi} \frac{z^{\prime}(2 \pi) \cdot\left(z^{\prime}(0)\right)^{\perp}(t-2 \pi)+R_{1}(t, \tau, z)}{\left|z^{\prime}(2 \pi)\right|^{2}(t-2 \pi)^{2}+\left|z^{\prime}(0)\right|^{2} \tau^{2}-2 \tau(t-2 \pi)\left|z^{\prime}(0)\right|\left|z^{\prime}(2 \pi)\right| \cos \beta+R_{2}(t, \tau, z)} .
\end{aligned}
$$

Die Restterme $R_{1}, R_{2}$ lassen sich durch $z^{(j)}, j=0,1,2$, ausdrücken und man erhält

$$
\left|R_{1}(t, \tau, z)\right| \leq C \delta^{2}\|z\|_{X}^{2},\left|R_{2}(t, \tau, z)\right| \leq C \delta^{3}\|z\|_{X}^{2}
$$

mit einer von $z$ unabhängigen Konstanten $C$. Damit ist die Existenz von $\delta>0$ mit der Eigenschaft (5.15) gezeigt. Aufgrund der Wahl von $R$ und $\delta$ erhält man für alle $z \in B\left[z_{0}, R\right]$ und $(t, \tau) \in[0, \delta] \times(0, \delta]$

$$
\begin{aligned}
|f(2 \pi-t, \tau, z)| & \leq 2|\tilde{f}(2 \pi-t, \tau, z)| \\
& \leq \frac{2}{\tau} k_{z}\left(\frac{t}{\tau}\right) \\
& \leq \frac{4}{\tau} k_{z_{0}}\left(\frac{t}{\tau}\right) .
\end{aligned}
$$


Desweiteren ist für

$$
f(t, \tau, z)=\frac{1}{\pi} \frac{z^{\prime}(\tau)^{\perp} \cdot[z(t)-z(\tau)]}{|z(t)-z(\tau)|^{2}}
$$

der Term

$$
\frac{\partial f}{\partial z}(t, \tau, z, h)
$$

durch eine integrierbare Funktion mal $\|h\|_{X}^{1}$ (gleichmäßig für $z \in B\left[z_{0}, R\right]$ ) abzuschätzen. Es gilt

$$
\begin{aligned}
\frac{\partial f}{\partial z}(t, \tau, z, h) & =f_{1}(t, \tau, z, h)+f_{2}(t, \tau, z, h) \text { mit } \\
f_{1}(t, \tau, z, h) & =\frac{1}{\pi} \frac{h^{\prime}(\tau)^{\perp} \cdot[z(t)-z(\tau)]+z^{\prime}(\tau)^{\perp} \cdot[h(t)-h(\tau)]}{|z(t)-z(\tau)|^{2}}, \\
f_{2}(t, \tau, z, h) & =\frac{-2}{\pi} \frac{z^{\prime}(\tau)^{\perp} \cdot[z(t)-z(\tau)]}{|z(t)-z(\tau)|^{2}} \frac{[z(t)-z(\tau)] \cdot[h(t)-h(\tau)]}{|z(t)-z(\tau)|^{2}} \\
& =-2 f(t, \tau, z) \frac{[z(t)-z(\tau)] \cdot[h(t)-h(\tau)]}{|z(t)-z(\tau)|^{2}} .
\end{aligned}
$$

Der Summand $f_{2}$ ist Produkt aus einem Mellin-Kern und einem Ausdruck, der für $z \in$ $B\left[z_{0}, R\right]$ durch $C\|h\|_{X}$ beschränkt bleibt. Für den ersten Summanden liefert eine Taylorentwicklung

$$
\begin{gathered}
\frac{1}{\pi} \frac{h^{\prime}(0)^{\perp} \tau \cdot\left[z^{\prime}(2 \pi)(t-2 \pi)-z^{\prime}(0) \tau\right]+z^{\prime}(0)^{\perp} \tau \cdot\left[h^{\prime}(2 \pi)(t-2 \pi)-h^{\prime}(0) \tau\right]+O\left(\delta^{2}\right)}{\left|z^{\prime}(2 \pi)\right|^{2}(t-2 \pi)^{2}+\left|z^{\prime}(0)\right|^{2} \tau^{2}-2(t-2 \pi) \tau z^{\prime}(2 \pi) \cdot z^{\prime}(0)+O\left(\delta^{2}\right)} \\
=\frac{1}{\pi} \frac{\left(h^{\prime}(0)^{\perp} \cdot z^{\prime}(2 \pi)+z^{\prime}(0)^{\perp} \cdot h^{\prime}(2 \pi)\right)(t-2 \pi) \tau+O\left(\delta^{2}\right)}{\left|z^{\prime}(2 \pi)\right|^{2}(t-2 \pi)^{2}+\left|z^{\prime}(0)\right|^{2} \tau^{2}-2(t-2 \pi) \tau z^{\prime}(2 \pi) \cdot z^{\prime}(0)+O\left(\delta^{2}\right)} .
\end{gathered}
$$

Der wesentliche Teil hat wiederum die Form

$$
\frac{C(z, h)(2 \pi-t) \tau}{a^{2}(t-2 \pi)^{2}+b^{2} \tau^{2}-2 \tau(t-2 \pi) a b \cos \beta} .
$$

Dabei sind die Konstanten $a, b$ von $h$ unabhängig, hängen aber von $z$ stetig ab. Die von $z$ und $h$ abhängende Konstante $C(z, h)$ genügt einer Abschätzung von der Form $|C(z, h)| \leq$ $\|h\|_{X}^{1} C\left(z_{0}, R\right)$. Damit lässt sich ganz analog durch Wahl von $R$ und anschließender Wahl von $\delta$ eine Majoranteneigenschaft bei den Ecken gewinnen, s.d. schließlich gilt

$$
\left|\frac{\partial f}{\partial z}(t, \tau, z, h)\right| \leq g(t, \tau)\|h\|_{X}^{1},(t, \tau) \in Q, z \in B\left[z_{0}, R\right],
$$


wobei $\sup _{0<t<2 \pi} \int_{0}^{2 \pi} g(t, \tau) d \tau<\infty$.

3. Weil im zweiten Teil des Beweises gleich allgemeiner argumentiert wurde, ist nur noch $\frac{\partial^{2}}{\partial z^{2}} f(t, \tau, z, h)$ abzuschätzen. Es gilt

$$
\begin{aligned}
\frac{\partial^{2} f}{\partial z^{2}}(t, \tau, z, h) & =\frac{\partial}{\partial z}\left(f_{1}+f_{2}\right)(t, \tau, z, h) \\
& =\sum_{j=1}^{4} g_{j}(t, \tau, z, h)
\end{aligned}
$$

mit den Funktionen

$$
\begin{aligned}
g_{1}(t, \tau, z, h) & =\frac{1}{\pi} \frac{2 h^{\prime}(\tau)^{\perp} \cdot[h(t)-h(\tau)]}{|z(t)-z(\tau)|^{2}} \\
g_{2}(t, \tau, z, h) & =\frac{-2}{\pi} \frac{h^{\prime}(\tau)^{\perp} \cdot[z(t)-z(\tau)]+z^{\prime}(\tau)^{\perp} \cdot[h(t)-h(\tau)]}{|z(t)-z(\tau)|^{2}} \frac{[z(t)-z(\tau)] \cdot[h(t)-h(\tau)]}{|z(t)-z(\tau)|^{2}} \\
& =-2 f_{1}(t, \tau, z, h) \frac{[z(t)-z(\tau)] \cdot[h(t)-h(\tau)]}{|z(t)-z(\tau)|^{2}}, \\
g_{3}(t, \tau, z, h) & =-2 \frac{\partial f}{\partial z}(t, \tau, z, h) \frac{[z(t)-z(\tau)] \cdot[h(t)-h(\tau)]}{|z(t)-z(\tau)|^{2}}, \\
g_{4}(t, \tau, z, h) & =-2 f(t, \tau, z)\left\{\frac{|h(t)-h(\tau)|^{2}}{|z(t)-z(\tau)|^{2}}-2 \frac{|[z(t)-z(\tau)] \cdot[h(t)-h(\tau)]|^{2}}{|z(t)-z(\tau)|^{4}}\right\} .
\end{aligned}
$$

Die Anteile mit Singularitäten vom Mellin-Typ wurden schon bei $f, f_{1}$ und $\frac{\partial f}{\partial z}$ behandelt. Alle anderen Faktoren und Summanden werden mit der Cauchy-Schwarzschen Ungleichung abgeschätzt. Es ist leicht abzulesen, dass sich alle Summanden $g_{j}(t, \tau, z, h)$ durch $\|h\|_{X}^{2}$ mal eine integrierbare Funktion abschätzen lassen, und zwar gleichmäßig für $z \in B\left[z_{0}, R\right]$.

\subsection{Die Fréchet - Ableitung des Fernfeldoperators}

In diesem Abschnitt wird die Fréchet-Differenzierbarkeit des Fernfeldoperators $\mathcal{F}$,

$$
\mathcal{F}: C_{2 \pi}(\mathbb{R}) \cap C^{2, \alpha}[0,2 \pi] \rightarrow L^{2}(\Omega), z \mapsto u_{\infty},
$$

der den Rand des Streugebiets auf das Fernfeld abbildet, nachgewiesen und die FréchetAbleitung $\mathcal{F}^{\prime}(z, h)$ durch die Lösung eines Randwertproblems charakterisiert. 
Um deutlich zu machen, auf welchem Rand die entsprechenden Funktionen auszuwerten sind, wird für eine zulässige Parametrisierung $z$ die Schreibweise

$$
\partial D_{z}=\{z(t) \mid 0 \leq t \leq 2 \pi\}
$$

vereinbart. Weiterhin wird (für ein Gebiet $G \subset \mathbb{R}^{2}$ ) für die Menge der von $G$ nach $\mathbb{C}$ beschränkten stetigen Funktionen abkürzend das Symbol $C_{b}(G)$ verwendet.

Da $\mathcal{F}$ Komposition verschiedener Operatoren ist, wird für die Anwendung der Kettenregel unter anderem die Differenzierbarkeit der Potenzialoperatoren benötigt. Diese wird durch den nachfolgenden Satz geklärt.

\section{Satz 5.11}

Seien $z_{0}$ eine zulässige Parametrisierung und $G \subset \mathbb{R}^{2}$ ein Gebiet mit $\bar{G} \subset \mathbb{R}^{2} \backslash \partial D_{0}$ sowie $R>0$ hinreichend klein. $P_{z}$ bezeichne den Potenzialoperator

$$
P_{z}: C_{2 \pi}(\mathbb{R}, \mathbb{C}) \rightarrow C_{b}(G)
$$

in parametrisierter Form

$$
\begin{aligned}
\left(P_{z} \psi\right)(x) & =\int_{0}^{2 \pi}\left(f_{K_{k}}-i \eta f_{S_{k}}\right)(x, \tau, z) \psi(\tau) d \tau, x \in G, \\
f_{K_{k}}(x, \tau, z) & :=-\frac{i k}{4} H_{0}^{(1)^{\prime}}(k|x-z(\tau)|) \frac{z^{\prime}(\tau)^{\perp} \cdot(x-z(\tau))}{|x-z(\tau)|}, \\
f_{S_{k}}(x, \tau, z) & :=\frac{i}{4} H_{0}^{(1)}(k|x-z(\tau)|)\left|z^{\prime}(\tau)\right| .
\end{aligned}
$$

Die Abbildung $z \mapsto P_{z}$ ist Fréchet-differenzierbar von $B\left(z_{0}, R\right) \subset U \rightarrow \mathcal{L}\left(C_{2 \pi}(\mathbb{R}, \mathbb{C}), C_{b}(G)\right)$ mit Ableitung

$$
\begin{gathered}
C_{2 \pi}(\mathbb{R}, \mathbb{C}) \cap C^{2, \alpha}[0,2 \pi] \rightarrow \mathcal{L}\left(C_{2 \pi}(\mathbb{R}, \mathbb{C}), C_{b}(G)\right), h \mapsto P^{\prime}(z, h), \\
\left(P^{\prime}(z, h) \psi\right)(x):=\int_{0}^{2 \pi} \frac{\partial}{\partial z}\left(f_{K_{k}}-i \eta f_{S_{k}}\right)(x, \tau, z, h) \psi(\tau) d \tau, x \in G,
\end{gathered}
$$

mit

$$
\begin{aligned}
\frac{\partial f_{S_{k}}}{\partial z}(x, \tau, z, h)= & \frac{i k}{4} H_{0}^{\prime}(k|x-z(\tau)|) \frac{[x-z(\tau)] \cdot(-h(\tau))}{|x-z(\tau)|}\left|z^{\prime}(\tau)\right| \\
& +\frac{i}{4} H_{0}(k|x-z(\tau)|) \frac{z^{\prime}(\tau) \cdot h^{\prime}(\tau)}{\left|z^{\prime}(\tau)\right|}
\end{aligned}
$$




$$
\begin{aligned}
\frac{\partial f_{K_{k}}}{\partial z}(x, \tau, z, h)= & \frac{i k}{4}\left\{\frac{k H_{0}^{\prime \prime}(k|x-z(\tau)|)}{|x-z(\tau)|}-\frac{H_{0}^{\prime}(k|x-z(\tau)|)}{|x-z(\tau)|^{2}}\right\} \times \\
& \times \frac{[x-z(\tau)] \cdot h(\tau)}{|x-z(\tau)|} z^{\prime}(\tau)^{\perp} \cdot(x-z(\tau)) \\
& -\frac{i k}{4} \frac{H_{0}^{\prime}(k|x-z(\tau)|)}{|x-z(\tau)|}\left\{h^{\prime}(\tau)^{\perp} \cdot(x-z(\tau))-z^{\prime}(\tau)^{\perp} \cdot h(\tau)\right\} .
\end{aligned}
$$

Beweis: Vergleiche Bemerkung 5.5 zum Satz 5.2.

Wir erklären die parametrisierten Einfach- und Doppelschichtpotenziale

$$
\begin{aligned}
& \left(E_{k}(z) \varphi\right)(x)=\int_{0}^{2 \pi} f_{S_{k}}(x, \tau, z)[\varphi(\tau)-\varphi(0)] d \tau, x \notin \partial D_{z}, \\
& \left(D_{k}(z) \psi\right)(x)=\int_{0}^{2 \pi} f_{K_{k}}(x, \tau, z)[\psi(\tau)-\psi(0)] d \tau, x \notin \partial D_{z} .
\end{aligned}
$$

Satz 5.12 Für die Potenziale $u(x)=\left(E_{k}(z) \varphi\right)(x)$ und $v(x)=\left(D_{k}(z) \psi\right)(x)$ sowie deren Ableitungen $u^{\prime}(x)=\left(E_{k}^{\prime}(z, h) \varphi\right)(x), v^{\prime}(x)=\left(D_{k}^{\prime}(z, h) \psi\right)(x)$ mit Dichten $\varphi \in C_{2 \pi}^{0, \alpha}(\mathbb{R}, \mathbb{C})$, $\psi \in C_{2 \pi}^{1, \alpha}(\mathbb{R}, \mathbb{C})$ gelten die Sprungbeziehungen

$$
\begin{aligned}
& u_{ \pm}^{\prime}(z(t))=\frac{1}{2}\left(S_{k}^{\prime}(z, h) \varphi\right)(t)-\operatorname{grad} u_{ \pm}(z(t)) \cdot h(t), 0<t<2 \pi, \\
& v_{ \pm}^{\prime}(z(t))=\frac{1}{2}\left(K_{k}^{\prime}(z, h) \psi\right)(t)-\operatorname{grad} v_{ \pm}(z(t)) \cdot h(t), 0<t<2 \pi .
\end{aligned}
$$

Beweis: Vergleiche [22, Theorem 3.2]. In den Beweis gehen lediglich Sprungbeziehungen und Abbildungseigenschaften für Einfach- und Doppelschichtpotenziale ein. Weil wir uns nur für Sprungbeziehungen außerhalb der Ecke interessieren, bleibt die Aussage des Satzes richtig.

Mit dem Restriktionsoperator $R_{z}: C\left(\mathbb{R}^{2}\right) \rightarrow C_{2 \pi}[0,2 \pi]$,

$$
\left(R_{z} f\right)(t):=-2 f(z(t)), 0 \leq t \leq 2 \pi,
$$

und dem Randintegraloperator

$$
A_{z}=K_{k}(z)-i \eta S_{k}(z)+\gamma_{k}(z)
$$


hat das zur einfallenden Welle $u^{i}$ am schallweichen Hindernis $D_{z}$ gestreute Feld $u^{s}$ die Form

$$
u^{s}(x)=\left(P_{z}\left(I+A_{z}\right)^{-1} R_{z} u^{i}\right)(x), x \in \mathbb{R}^{2} \backslash \overline{D_{z}} .
$$

Das zugehörige Fernfeld $u_{\infty}$ ergibt sich als Fernfeld des Potenzials $P_{z}$ mit Dichte $\left(I+A_{z}\right)^{-1} R_{z} u^{i}$, d.h.

$$
u_{\infty}=P_{z, \infty}\left(I+A_{z}\right)^{-1} R_{z} u^{i}
$$

Nach der Kettenregel sind

$$
\begin{aligned}
B\left(z_{0}, R\right) \subset U \rightarrow C^{2}\left(\mathbb{R}^{2} \backslash \overline{D_{z_{0}}}\right), & z \mapsto u^{s}, \\
B\left(z_{0}, R\right) \subset U \rightarrow L^{2}(\Omega), & z \mapsto u_{\infty}
\end{aligned}
$$

Fréchet-differenzierbar mit Ableitung

$$
C_{2 \pi}(\mathbb{R}, \mathbb{C}) \cap C^{2, \alpha}([0,2 \pi], \mathbb{C}) \rightarrow C^{2}\left(\mathbb{R}^{2} \backslash \overline{D_{z_{0}}}\right), h \mapsto v=v_{1}+v_{2}+v_{3},
$$

beziehungsweise $h \mapsto v_{\infty}$, wobei

$$
\begin{aligned}
& v_{1}=P^{\prime}(z, h)\left(I+A_{z}\right)^{-1} R_{z} u^{i}, \\
& v_{2}=-P_{z}\left(I+A_{z}\right)^{-1} A^{\prime}(z, h)\left(I+A_{z}\right)^{-1} R_{z} u^{i}, \\
& v_{3}=P_{z}\left(I+A_{z}\right)^{-1} R^{\prime}(z, h) u^{i}
\end{aligned}
$$

gilt und $v_{\infty}$ das Fernfeld von $v$ darstellt.

Als nächstes soll $v$ durch ein Randwertproblem charakterisiert werden. $v_{2}$ und $v_{3}$ lösen die Helmholtzgleichung in $\mathbb{R}^{2} \backslash \bar{D}$ und erfüllen die Sommerfeldsche Ausstrahlungsbedingung. Da außerhalb des Randes die Fréchet-Differenziation und gewöhnliche Differenziation nach $x$ vertauschbar sind, erfüllt auch $v_{1}$ diese Bedingungen. Die Randwerte von $v$ sind zu bestimmen. $v_{3}$ löst das äußere Dirichletproblem zu den Randwerten $\frac{1}{2} R^{\prime}(z, h) u^{i}$, d.h.

$$
\begin{aligned}
v_{3}(z(t)) & =\frac{1}{2}\left(R^{\prime}(z, h) u^{i}\right)(z(t)) \\
& =-\operatorname{grad} u^{i}(z(t)) \cdot h(t) \\
& =-\operatorname{grad} u(z(t)) \cdot h(t)+\operatorname{grad} u^{s}(z(t)) \cdot h(t) \\
& =-\nu(z(t)) \frac{\partial u}{\partial \nu}(z(t)) \cdot h(t)+\operatorname{grad} u^{s}(z(t)) \cdot h(t), 0<t<2 \pi .
\end{aligned}
$$

Dabei wurden die Gleichungen $u=u^{i}+u^{s}$ und $u(z(t))=0, t \in[0,2 \pi]$, verwendet. Insbesondere wird aus Gleichung (5.17) die Regularität

$$
v_{3} \in C^{2}\left(\mathbb{R}^{2} \backslash \bar{D}_{z}\right) \cap C\left(\mathbb{R}^{2} \backslash D_{z}\right)
$$


deutlich. Desgleichen ergibt sich aufgrund der Definition als kombiniertes Einfach- und Doppelschichtpotenzial

$$
v_{2}(z(t))=-\frac{1}{2}\left(A^{\prime}(z, h)\left(I+A_{z}\right)^{-1} R_{z} u^{i}\right)(z(t))=\frac{1}{2}\left(A^{\prime}(z, h)\left(I+A_{z}\right)^{-1} R_{z} u^{s}\right)(z(t)) .
$$

Aufgrund der Abbildungseigenschaft $A^{\prime}(z, h): C_{2 \pi} \rightarrow C_{2 \pi}$ sind die Randwerte von $v_{2}$ ebenfalls stetig.

Es verbleibt $v_{1}$ zu untersuchen. Es gilt $v_{1}(z(t))=\left(P^{\prime}(z, h) \varphi\right)_{+}(z(t))$ mit der Dichte $\varphi=\left(I+A_{z}\right)^{-1} R_{z} u^{i}$. Es gilt

$$
\begin{aligned}
\left(P^{\prime}(z, h) \varphi\right)(x)= & \int_{0}^{2 \pi} \frac{\partial}{\partial z}\left(f_{K_{k}}-i \eta f_{S_{k}}\right)(x, \tau, z, h)[\varphi(\tau)-\varphi(0)] d \tau \\
& +\varphi(0) \int_{0}^{2 \pi} \frac{\partial}{\partial z}\left(f_{K_{k}}-f_{K_{0}}-i \eta f_{S_{k}}\right)(x, \tau, z, h) d \tau, x \in \mathbb{R}^{2} \backslash \overline{D_{z}} .
\end{aligned}
$$

Weil die Dichte $\varphi$ auf jedem abgeschlossenen Teilstück von $] 0,2 \pi\left[C^{1, \alpha}\right.$-glatt ist, erhält man unter Verwendung von Satz 5.12 für $0<t<2 \pi$

$$
\begin{aligned}
& \left(P^{\prime}(z, h) \varphi\right)_{+}(z(t)) \\
= & \frac{1}{2}\left(\left(K_{k}-i \eta S_{k}\right)^{\prime}(z, h) \varphi\right)(t)-\operatorname{grad}\left(D_{k}(z)-i \eta E_{k}(z)\right) \varphi_{+}(z(t)) \cdot h(t) \\
& +\varphi(0)\left[\frac{1}{2}\left(\left(K_{k}-K_{0}-i \eta S_{k}\right)^{\prime}(z, h) \mathbf{1}\right)(t)-\operatorname{grad}\left(D_{k}(z)-D_{0}(z)-i \eta E_{k}(z)\right) \mathbf{1}_{+}(z(t)) \cdot h(t)\right] \\
= & \frac{1}{2}\left(A^{\prime}(z, h) \varphi\right)(t)-\operatorname{grad}\left(D_{k}(z)-i \eta E_{k}(z)\right) \varphi_{+}(z(t)) \cdot h(t) \\
& -\varphi(0) \operatorname{grad}\left(D_{k}(z)-D_{0}(z)-i \eta E_{k}(z)\right) 1_{+}(z(t)) \cdot h(t) \\
= & -\frac{1}{2}\left(A^{\prime}(z, h)\left(I+A_{z}\right)^{-1} R_{z} u^{s}\right)(z(t))-\operatorname{grad} u^{s}(z(t)) \cdot h(t) .
\end{aligned}
$$

Durch Zusammenfügen der drei Randbedingungen für $v_{j}, j=1,2,3$, erhält man als Randbedingung für $v=v_{1}+v_{2}+v_{3}$ schließlich

$$
v(z(t))=-\nu(z(t)) \frac{\partial u}{\partial \nu}(z(t)) \cdot h(t), 0<t<2 \pi .
$$

Es wurde unter anderem festgestellt, dass $v_{2}$ und $v_{3}$ bis in den Rand von $D_{z}$ stetig sind. Damit lässt sich die Fréchet-Ableitung des Fernfeldoperators durch den nachfolgenden Satz charakterisieren. 
Satz 5.13 Die Fréchet-Ableitung $\mathcal{F}^{\prime}(z, h)$ ist gegeben durch das Fernfeld $v_{\infty}$, wobei $v$ das Randwertproblem

$$
\begin{aligned}
\triangle v+k^{2} v & =0 \text { in } \mathbb{R}^{2} \backslash \overline{D_{z}} \\
v(z(t)) & =-\nu(z(t)) \frac{\partial u}{\partial \nu}(z(t)) \cdot h(t), 0<t<2 \pi,
\end{aligned}
$$

löst derart, dass $v-v_{1}$ stetig in $\mathbb{R}^{2} \backslash D_{z}$ ist.

Ausgewertet wird obige Fréchet-Ableitung dadurch, dass für $b:=v-v_{1}$ das Randwertproblem

$$
\begin{aligned}
\triangle b+k^{2} b & =0 \text { in } \mathbb{R}^{2} \backslash \bar{D} \\
b(z(t)) & =-\operatorname{grad} u^{i}(z(t)) \cdot h(t)-\frac{1}{2}\left(A^{\prime}(z, h)\left(I+A_{z}\right)^{-1} R_{z} u^{i}\right)(z(t)), 0 \leq t \leq 2 \pi,
\end{aligned}
$$

gelöst wird und

$$
v_{\infty}=b_{\infty}+v_{1, \infty}
$$

zu setzen ist.

Mit der Asymptotik (2.4) sowie

$$
H_{0}^{\prime \prime}(t)=-H_{0}^{(1)}(t)\left\{1+O\left(\frac{1}{t}\right)\right\}, t \rightarrow \infty,
$$

erhält man für das Fernfeld $v_{1, \infty}$ von $v_{1}$ die Darstellung

$$
\begin{aligned}
& \left(v_{1, \infty}\right)(\hat{x}) \\
& =\frac{e^{-i \frac{\pi}{4}}}{\sqrt{8 \pi k}} \int_{0}^{2 \pi}\left[k h^{\prime}(\tau)^{\perp} \cdot \hat{x}-i k^{2} z^{\prime}(\tau)^{\perp} \cdot \hat{x} \hat{x} \cdot h(\tau)+\right. \\
& \left.\quad+\eta\left\{\frac{z^{\prime}(\tau) \cdot h^{\prime}(\tau)}{\left|z^{\prime}(\tau)\right|}-i k \hat{x} \cdot h(\tau)\left|z^{\prime}(\tau)\right|\right\}\right] e^{-i k \hat{x} \cdot z(\tau)} \varphi(z(\tau)) d \tau, \quad|\hat{x}|=1,
\end{aligned}
$$

mit der Dichte

$$
\varphi=\left(I+A_{z}\right)^{-1} R_{z} u^{i}
$$

Das Ergebnis (5.20) ist auch plausibel, denn formales Differenzieren nach $z$ in der parametrisierten Form von (4.19) liefert das gleiche Resultat.

Satz 5.13 ermöglicht nun die Auswertung der Fréchet-Ableitung von $\mathcal{F}$. Eine ausführliche Beschreibung des inversen Verfahrens und dessen numerische Umsetzung erfolgt im Abschnitt 6.1] des nächsten Kapitels. 


\section{Kapitel 6}

\section{Numerische Ergebnisse beim inversen Streuproblem}

In diesem Kapitel wird dargestellt, wie sich mit einem regularisierten Newtonverfahren der in Abschnitt 5.3 gewonnene Satz 5.13 zur Rekonstruktion des Streugebietes einsetzen lässt. Dabei wird im Abschnitt 6.1 das Verfahren allgemein erläutert. Im zweiten Teil wird eine Modifizierung im Tikhonov-Funktional vorgenommen und ein zu lösendes Gleichungssystem hergeleitet. Im Abschnitt 6.3 wird auf den Operator $A^{\prime}(z, h)$ und dessen numerische Behandlung eingegangen. In den Abschnitten 6.4 bis 6.6 werden für verschiedene Testsituationen numerische Ergebnisse vorgestellt. Dabei wurde sowohl für eine einfallende ebene Welle, als auch für mehrere Messdaten gerechnet. Desweiteren wurde auch der Fall eines eingeschränkten Messbereichs untersucht.

\subsection{Newtonverfahren und Regularisierung}

Die zu lösende Operatorgleichung

$$
\mathcal{F}(z)=u_{\infty}^{\text {meas }}
$$

für die Parametrisierung $z$ des unbekannten Streugebietes bei gegegeben Meßwerten $u_{\infty}^{\text {meas }}$, ist nichtlinear und schlecht gestellt.

Gleichung 6.1 wird durch die linearisierte Gleichung

$$
\mathcal{F}(z)+\mathcal{F}^{\prime}(z, h)=u_{\infty}^{\text {meas }}
$$


ersetzt, sodass sich bei geeigneter Startparametrisierung $z_{0}$ ein Iterationsverfahren

$$
\begin{aligned}
\mathcal{F}^{\prime}\left(z_{p}, h_{p}\right) & =u_{\infty}^{\text {meas }}-\mathcal{F}\left(z_{p}\right) \\
z_{p+1} & =z_{p}+h_{p}, p=0,1,2, \ldots,
\end{aligned}
$$

ergibt.

Die linearisierte Gleichung (6.3) soll nun auf einem endlichdimensionalen Unterraum $Q_{N}=\operatorname{span}\left\{q_{1}, \ldots, q_{N}\right\} \subset X$ näherungsweise gelöst werden. Dabei wird das semidiskrete Problem

$$
\sum_{j=1}^{N} a_{j}^{(p)} \mathcal{F}^{\prime}\left(z_{p}, q_{j}\right)=u_{\infty}^{\text {meas }}-\mathcal{F}\left(z_{p}\right)
$$

zur Ermittlung von

$$
h_{p}=\sum_{j=1}^{N} a_{j}^{(p)} q_{j} \in Q_{N}
$$

ersetzt durch ein volldiskretes Problem. Durch Wahl von $M$ Kollokationspunkten $\hat{x}_{1}, \ldots, \hat{x}_{M} \in$ $\Omega$ ergibt sich das lineare Gleichungssystem

$$
\sum_{j=1}^{N} a_{j}^{(p)} \mathcal{F}^{\prime}\left(z_{p}, q_{j}\right)\left(\hat{x}_{i}\right)=u_{\infty}^{\text {meas }}\left(\hat{x}_{i}, d\right)-\mathcal{F}\left(z_{p}\right)\left(\hat{x}_{i}\right), \quad i=1, \ldots, M
$$

für die $N$ Unbekannten $a_{1}^{(p)}, \ldots, a_{N}^{(p)} \in \mathbb{R}$. Dann ist

$$
h_{p}=\sum_{j=1}^{N} a_{j}^{(p)} q_{j} \in Q_{N}
$$

eine Näherungslösung von (6.3).

Da sich die Schlechtgestelltheit von (6.1) auf (6.4) vererbt, macht es keinen Sinn, das Gleichungssystem exakt zu lösen.

Grundlage für ein numerisches Verfahren zur Lösung von (6.4) bildet der nachfolgende Satz zur Tikhonov-Regularisierung.

Satz 6.1 Seien $X, Y$ Hilberträume, $A: X \rightarrow Y$ linear, kompakt und $\alpha>0$. Dann gilt:

a) Für A existiert ein singuläres System $\left(\mu_{n}, \varphi_{n}, g_{n}\right)$. 
b) $\alpha I+A^{*} A: X \rightarrow X$ ist bijektiv und beschränkt invertierbar. Insbesondere ist $R_{\alpha}=\left(\alpha I+A^{*} A\right)^{-1} A^{*}$ aus $\mathcal{L}(Y, X)$.

c) Die für $f \in Y$ eindeutig bestimmte Lösung $\varphi_{\alpha} \in X$ der Gleichung

$$
\alpha \varphi_{\alpha}+A^{*} A \varphi_{\alpha}=A^{*} f
$$

hat die Darstellung

$$
\varphi_{\alpha}=\sum_{n=1}^{\infty} \frac{\mu_{n}}{\alpha+\mu_{n}^{2}}\left(f, g_{n}\right) \varphi_{n}
$$

d) Die Optimierungsaufgabe $\min _{\varphi \in X} J(\varphi)$ für das Tikhonov-Funktional

$$
J(\varphi)=\|A \varphi-f\|^{2}+\alpha\|\varphi\|^{2}
$$

besitzt genau eine Lösung $\varphi_{\alpha}$. Diese wird durch (6.5) gegeben.

e) Zusätzlich sei $A$ injektiv und für den Wertebereich gelte $\overline{A(X)}=Y$. Für $\delta>0$, $f \in A(X), f^{\delta} \in Y$ mit $\left\|f-f^{\delta}\right\| \leq \delta<\left\|f^{\delta}\right\|$ existiert genau ein $\alpha=\alpha(\delta)>0$ so, dass gilt

$$
\left\|A R_{\alpha(\delta)} f^{\delta}-f^{\delta}\right\|=\delta .
$$

Für dieses $\alpha(\delta)$ konvergiert (mit $\delta \rightarrow 0$ ) die Lösung $\varphi_{\alpha(\delta)}$ der Gleichung

$$
R_{\alpha(\delta)} \varphi_{\alpha(\delta)}=f^{\delta}
$$

gegen die Lösung von $A \varphi=f$.

Beweis: siehe [7, Theorem 4.13 und 4.16]

Da die Matrixeinträge $\mathcal{F}\left(z_{p}, q_{j}\right)\left(\hat{x}_{i}\right)$ und die rechte Seite $u_{\infty}^{\text {meas }}\left(\hat{x}_{i}, d\right)-\mathcal{F}\left(z_{p}\right)\left(\hat{x}_{i}\right)$ des linearen Gleichungssystems (6.4) komplexwertig sind, wir aber eine reelle Lösung $a_{1}^{(p)}, \ldots, a_{N}^{(p)}$ suchen, ist (6.4) ein Gleichungssystem mit $2 M$ Gleichungen und $N$ Unbekannten. Für $2 M>N$ ist dieses überbestimmt, sodass das lineare Ausgleichsproblem nach der Methode der kleinsten Quadrate zusammen mit Satz 6.1 auf das Problem

$$
\left(\alpha_{p} I+C^{(p)^{t}} C^{(p)}\right) a^{(p)}=C^{(p)^{t}} g^{(p)}
$$


führt, wobei $\alpha_{p}>0$ ein geeigneter Regularisierungsparameter ist und für die Komponenten der Matrix $C^{(p)} \in \mathbb{R}^{2 M \times N}$, beziehungsweise der rechten Seite $g^{(p)} \in \mathbb{R}^{2 M}$, gilt

$$
\begin{array}{rlrl}
c_{i j}^{(p)} & =\operatorname{Re}\left(\mathcal{F}^{\prime}\left(z_{p}, q_{j}\right)\left(\hat{x}_{i}\right)\right), & & i=1, \ldots, M, j=1, \ldots, N, \\
c_{i j}^{(p)} & =\operatorname{Im}\left(\mathcal{F}^{\prime}\left(z_{p}, q_{j}\right)\left(\hat{x}_{i-M}\right)\right), & & i=M+1, \ldots, 2 M, j=1, \ldots, N, \\
C^{(p)} & =\left(c_{i j}^{(p)}\right)_{\substack{1 \leq i \leq 2 M \\
1 \leq j \leq N}} \in \mathbb{R}^{2 M \times N}, & \\
g_{i}^{(p)} & =\operatorname{Re}\left(u_{\infty}^{\text {meas }}\left(\hat{x}_{i}, d\right)-\mathcal{F}\left(z_{p}\right)\left(\hat{x}_{i}\right)\right), & & i=1, \ldots, M, \\
g_{i}^{(p)} & =\operatorname{Im}\left(u_{\infty}^{\text {meas }}\left(\hat{x}_{i-M}, d\right)-\mathcal{F}\left(z_{p}\right)\left(\hat{x}_{i-M}\right)\right), & i=M+1, \ldots, 2 M, \\
g^{(p)} & =\left(g_{i}^{(p)}\right)_{1 \leq i \leq 2 M} \in \mathbb{R}^{2 M}, & \\
a^{(p)} & =\left(a_{j}^{(p)}\right)_{1 \leq j \leq N} \in \mathbb{R}^{N} . &
\end{array}
$$

Ein Iterationsschritt für das Newtonverfahren (6.3) lässt sich wie folgt beschreiben:

1. Für eine gegebene Parametrisierung $z_{p}$ wird die zum Operator $I+A\left(z_{p}\right)$ gemäß (5.16) gehörige Matrix (3.20) mit den Kernen (4.31) und deren $L U$-Zerlegung ermittelt.

Für eine einfallende ebene Welle $u^{i}(\cdot, d)$ ist die rechte Seite gemäß (4.30) zu bestimmen. Die Lösung $\varphi_{i}^{(n)}, i=0, \ldots, 2 n-1$, des zugehörigen linearen Gleichungssystems (3.20) sind Näherungswerte für $\varphi(z(w(i \pi / n)))$.

2. Berechne $\mathcal{F}\left(z_{p}\right)\left(\hat{x}_{i}\right), i=1, \ldots, M$, gemäß (4.32) und hieraus $g^{(p)}$.

3. Berechne $\mathcal{F}^{\prime}\left(z_{p}, h\right)$ für $h=q_{1}, \ldots, q_{N}$ : Dazu sind zu den $N$ rechten Seiten gemäß (5.18) und der unter 1. gewonnenen $L U$-Zerlegung die Fernfelder dieser $N$ direkten Probleme an den Kollokationspunkten $\hat{x}_{1}, \ldots, \hat{x}_{M}$, auszuwerten. Unter Berücksichtigung der Fernfelder der singulären Anteile (siehe Gleichungen (5.19) - (5.20) ergeben sich die Werte $\mathcal{F}^{\prime}\left(z_{p}, q_{j}\right)\left(\hat{x}_{i}\right)$. Stelle die Matrix $C^{(p)}=\left(c_{i j}^{(p)}\right)_{\substack{1 \leq i \leq 2 M \\ 1 \leq j \leq N}}^{\substack{5 \\ 1 \leq 0}}$.

4. Löse (6.6) zur Ermittlung von $a_{j}^{(p)}, j=1, \ldots, N$ und bilde $z_{p+1}=z_{p}+\sum_{j=1}^{N} a_{j}^{(p)} q_{j}$.

5. Bilde das Residuum

$$
\text { Res }:=\frac{\sum_{i=1}^{M}\left|\mathcal{F}\left(z_{p+1}\right)\left(\hat{x}_{i}\right)-u_{\infty}^{\text {meas }}\left(\hat{x}_{i}\right)\right|^{2}}{\sum_{i=1}^{M}\left|u_{\infty}^{\text {meas }}\left(\hat{x}_{i}\right)\right|^{2}} .
$$

Breche ab, falls Res $<\varepsilon$. Andernfalls erhöhe $p$ um 1 und fahre fort mit Schritt 1. 
In jedem Newton-Schritt sind demzufolge $N+1$ direkte Probleme zu lösen. Die dabei zu invertierende Matrix $I+A\left(z_{p}\right)$ ist stets die gleiche.

Es bleibt die Frage nach der Wahl von passenden Ansatzräumen $Q_{N}$ und der benötigten Fernfelddaten $u_{\infty}^{\text {meas }}$. Die Fernfelddaten werden synthetisch erzeugt, wobei zur Vermeidung von „Inverse Crimes“ beim Vorwärtslöser mit einer anderen Stützstellenzahl $n$, einem anderen Kopplungsparameter $\eta$ und mit einer anderen Substitutionsfunktion $w$ gearbeitet wird. Desweiteren darf die eigentlich unbekannte Parametrisierung $z$ nicht im Ansatzraum $Q_{N}$ enthalten sein.

Abschließend wird angemerkt, dass bei sternförmigen Gebieten die Ableitung des Fernfeldoperators zum Dirichlet-Problem sowohl injektiv ist, als auch dichten Wertebereich hat. Das für glatte Ränder bekannte Resultat (siehe Kreß [30]) lässt sich auf den hier vorliegenden Fall übertragen. Die Basiselemente $q_{j}$ von $Q_{N}$ lassen sich in der Form

$$
q_{j}(t)=r_{j}(t)\left(\begin{array}{c}
\cos t \\
\sin t
\end{array}\right), 0 \leq t \leq 2 \pi, j=1, \ldots, N,
$$

schreiben. Dabei sind die Radialfunktionen $r_{j}$ linear unabhängig.

\subsection{Modifizierung im Tikhonov-Funktional}

In diesem Abschnitt wird beschrieben, wie sich durch eine zusätzliche „Bestrafung“ von Ableitungen der Störung im Tikhonov-Funktional das zu lösende Gleichungssystem verändert. Dabei wird ein Ansatz wie in Hohage-Schormann [22] gewählt. Zusätzlich wird davon ausgegangen, dass Messdaten für $D$ Einfallsrichtungen vorliegen, das heißt gegeben sind

$$
u_{\infty}^{\text {meas }}\left(\hat{x}_{i}, d_{l}\right), 1 \leq i \leq M, 1 \leq l \leq D .
$$

Das Tikhonov-Funktional wird ersetzt durch

$$
J\left(h_{p}\right)=\sum_{l=1}^{D}\left\|\mathcal{F}^{\prime}\left(z_{p}, h_{p}\right)\left(\cdot, d_{l}\right)-\left[u_{\infty}^{\text {meas }}-\mathcal{F}\left(z_{p}\right)\right]\left(\cdot, d_{l}\right)\right\|_{L^{2}(\Omega)}^{2}+\alpha_{p}\left\|h_{p}\right\|_{H^{s}[0,2 \pi]}^{2} .
$$

Dabei ist $s \in \mathbb{N}$ und $\|\cdot\|_{H^{s}}$ eine geeignete Sobolev-Norm, etwa

$$
\|\psi\|_{H^{s}[0,2 \pi]}^{2}=\|r\|_{L^{2}[0,2 \pi]}^{2}+\left\|r^{(s)}\right\|_{L^{2}[0,2 \pi]}^{2}, \psi(t)=r(t)(\cos t, \sin t)^{t} .
$$


Mit den Approximationen

$$
\begin{aligned}
\|\psi\|_{L^{2}(\Omega)}^{2} & \approx \frac{1}{M} \sum_{i=1}^{M}\left|\psi\left(\hat{x}_{i}\right)\right|^{2} \\
\|\psi\|_{H^{s}[0,2 \pi]}^{2} & \approx \frac{1}{K} \sum_{i=1}^{K}\left(\left|r\left(\hat{t}_{i}\right)\right|^{2}+\left|r^{(s)}\left(\hat{t}_{i}\right)\right|^{2}\right)
\end{aligned}
$$

hat das zu (6.7) zugehörige endlichdimensionale Problem die Form

$$
\begin{aligned}
& \text { Minimiere } \quad \sum_{l=1}^{D} \sum_{i=1}^{M}\left|\sum_{j=1}^{N} a_{j}^{(p)} \mathcal{F}^{\prime}\left(z_{p}, q_{j}\right)\left(\hat{x}_{i}, d_{l}\right)+\mathcal{F}\left(z_{p}\right)\left(\hat{x}_{i}, d_{l}\right)-u_{\infty}^{\text {meas }}\left(\hat{x}_{i}, d_{l}\right)\right|^{2} \\
& +\sum_{i=1}^{K}\left|\sqrt{\alpha_{p} \frac{M}{K}} \sum_{j=1}^{N} a_{j}^{(p)} r_{j}\left(\hat{t}_{i}\right)\right|^{2}+\sum_{i=1}^{K}\left|\sqrt{\alpha_{p} \frac{M}{K}} \sum_{j=1}^{N} a_{j}^{(p)} r_{j}^{(s)}\left(\hat{t}_{i}\right)\right|^{2}
\end{aligned}
$$

Dabei sind die $K$ Punkte $\hat{t}_{1}, \ldots, \hat{t}_{K}$ zur Auswertung der Sobolev-Norm aus $(0,2 \pi)$ in der Regel verschieden von $s_{i}^{(n)}, i=0, \ldots, 2 n-1$.

Das Minimierungsproblem (6.8) ist äquivalent $\mathrm{zu}$

$$
\text { Minimiere }\left\|C^{(p)} a^{(p)}-g^{(p)}\right\|_{2} \quad \text { beziehungsweise } C^{(p)^{t}} C^{(p)} a^{(p)}=C^{(p)^{t}} g^{(p)} .
$$

Die Komponenten der Matrix $C^{(p)} \in \mathbb{R}^{(2 M D+2 K) \times N}$ und die der Vektoren $a^{(p)} \in \mathbb{R}^{N}$ und $g^{(p)} \in \mathbb{R}^{2 M D+2 K}$ sind gegeben durch

$$
\begin{array}{rlrl}
c_{2 M(l-1)+i, j}^{(p)}=\operatorname{Re}\left(\mathcal{F}^{\prime}\left(z_{p}, q_{j}\right)\left(\hat{x}_{i}, d_{l}\right)\right), & & i=1, \ldots, M, j=1, \ldots, N, \\
c_{2 M(l-1)+i+M, j}^{(p)}=\operatorname{Im}\left(\mathcal{F}^{\prime}\left(z_{p}, q_{j}\right)\left(\hat{x}_{i}, d_{l}\right)\right), & & i=1, \ldots, D, \\
c_{i j}^{(p)}=\sqrt{\alpha_{p} M / K} r_{j}\left(\hat{t}_{i-2 M D}\right), & & i=1, \ldots, D, \\
& & i=2 M D+1, \ldots, 2 M D+K, \\
c_{i j}^{(p)}=\sqrt{\alpha_{p} M / K} r_{j}^{(s)}\left(\hat{t}_{i-2 M D-K}\right), & & j=1, \ldots, N, \\
& & i=2 M D+K+1, \ldots, 2 M D+2 K, \\
g_{2 M(l-1)+i}^{(p)}=\operatorname{Re}\left(u_{\infty}^{\text {meas }}\left(\hat{x}_{i}, d_{l}\right)-\mathcal{F}\left(z_{p}\right)\left(\hat{x}_{i}, d_{l}\right)\right), & i=1, \ldots, M, l=1, \ldots, D, \\
g_{2 M(l-1)+i+M}^{(p)}=\operatorname{Im}\left(u_{\infty}^{\text {meas }}\left(\hat{x}_{i}, d_{l}\right)-\mathcal{F}\left(z_{p}\right)\left(\hat{x}_{i}, d_{l}\right)\right), & i=1, \ldots, M, l=1, \ldots, D, \\
g_{i}^{(p)}=0, & & i=2 M D+1, \ldots, 2 M D+2 K, \\
\hat{t}_{i}=(i-1 / 2) 2 \pi / K, & i=1, \ldots, K .
\end{array}
$$




\subsection{Die rechte Seite der Fréchet - Ableitung}

Für die Auswertung der rechten Seite in (5.18) sind die zu $A^{\prime}(z, h)$ gehörenden Operatoren zu parametrisieren.

Zur vereinfachten Darstellung weichen die Notationen der Kerne von denen im Abschnitt 5.2 leicht ab. Für die Randintegraloperatoren

$$
S_{k}^{\prime}(z), K_{k}^{\prime}(z), K_{0}^{\prime}(z), \gamma_{k}^{\prime}(z): C_{2 \pi} \rightarrow C_{2 \pi}
$$

gelten die Darstellungen

$$
\begin{aligned}
\left(S_{k}^{\prime}(z, h) \varphi\right)(t) & =\int_{0}^{2 \pi} M_{k}^{\prime}(t, \tau, z, h)[\varphi(\tau)-\varphi(0)] d \tau, \\
\left(K_{k}^{\prime}(z, h) \varphi\right)(t) & =\int_{0}^{2 \pi} L_{k}^{\prime}(t, \tau, z, h)[\varphi(\tau)-\varphi(0)] d \tau, \\
\left(K_{0}^{\prime}(z, h) \varphi\right)(t) & =\int_{0}^{2 \pi} L_{0}^{\prime}(t, \tau, z, h)[\varphi(\tau)-\varphi(0)] d \tau, \\
\left(\gamma_{k}^{\prime}(z, h) \varphi\right)(t) & =\varphi(0) \int_{0}^{2 \pi}\left(L_{k}^{\prime}-L_{0}^{\prime}-i \eta M_{k}^{\prime}\right)(t, \tau, z, h) d \tau .
\end{aligned}
$$

Mit der abkürzenden Schreibweise $r(t, \tau)=|z(t)-z(\tau)|$ haben die zugehörigen Kerne die Form

$$
\begin{aligned}
M_{k}^{\prime}(t, \tau, z, h)= & -\frac{i k}{2} H_{1}^{(1)}(k r(t, \tau)) \frac{(z(t)-z(\tau)) \cdot(h(t)-h(\tau))}{r(t, \tau)}\left|z^{\prime}(\tau)\right| \\
& +\frac{i}{2} H_{0}^{(1)}(k r(t, \tau)) \frac{z^{\prime}(\tau) \cdot h^{\prime}(\tau)}{\left|z^{\prime}(\tau)\right|}, \\
L_{k}^{\prime}(t, \tau, z, h)= & \frac{i k}{2} H_{1}^{(1)}(k r(t, \tau)) \frac{h^{\prime}(\tau)^{\perp} \cdot(z(t)-z(\tau))+z^{\prime}(\tau)^{\perp} \cdot(h(t)-h(\tau))}{r(t, \tau)} \\
& +\frac{i}{2}\left\{k^{2} H_{0}^{(1)}(k r(t, \tau))-2 k \frac{H_{1}^{(1)}(k r(t, \tau))}{r(t, \tau)}\right\} \times \\
& \times \frac{(z(t)-z(\tau)) \cdot(h(t)-h(\tau))}{[r(t, \tau)]^{2}} z^{\prime}(\tau)^{\perp} \cdot(z(t)-z(\tau)), \\
L_{0}^{\prime}(t, \tau, z, h)= & \frac{1}{\pi} \frac{h^{\prime}(\tau)^{\perp} \cdot(z(t)-z(\tau))+z^{\prime}(\tau)^{\perp} \cdot(h(t)-h(\tau))}{[r(t, \tau)]^{2}} \\
& -\frac{2}{\pi} \frac{(z(t)-z(\tau)) \cdot(h(t)-h(\tau))}{[r(t, \tau)]^{4}} z^{\prime}(\tau)^{\perp} \cdot(z(t)-z(\tau)) .
\end{aligned}
$$


Dabei wurde die Ableitung $H_{0}^{\prime \prime}$ mit den Identitäten (2.1) und $H_{0}^{(1) \prime}=-H_{1}^{(1)}$ gemäß

$$
-H_{0}^{\prime \prime}(t)=\frac{1}{t} H_{0}^{\prime}(t)+H_{0}(t)=-\frac{1}{t} H_{1}^{(1)}(t)+H_{0}^{(1)}(t), t>0
$$

ersetzt. Die Abspaltung der logarithmischen Anteile führt auf

$$
\begin{aligned}
M_{k}^{\prime}(t, \tau, z, h) & =M_{k}^{\prime(1)}(t, \tau, z, h) \ln \left(4 \sin ^{2} \frac{t-\tau}{2}\right)+M_{k}^{\prime(2)}(t, \tau, z, h), \\
L_{k}^{\prime}(t, \tau, z, h) & =L_{k}^{\prime(1)}(t, \tau, z, h) \ln \left(4 \sin ^{2} \frac{t-\tau}{2}\right)+L_{k}^{\prime(2)}(t, \tau, z, h), \\
L_{0}^{\prime}(t, \tau, z, h) & =L_{0}^{\prime(1)}(t, \tau, z, h) \ln \left(4 \sin ^{2} \frac{t-\tau}{2}\right)+L_{0}^{\prime(2)}(t, \tau, z, h) .
\end{aligned}
$$

Entweder aus der Asymptotik von $H_{0}^{(1)}$ und $H_{1}^{(1)}$ oder durch direktes Differenzieren in den Darstellungen (4.22) - (4.25) ergeben sich die Kerne

$$
\begin{aligned}
M_{k}^{\prime(1)}(t, \tau, z, h)= & \frac{k}{2 \pi} J_{1}(k r(t, \tau)) \frac{(z(t)-z(\tau)) \cdot(h(t)-h(\tau))}{r(t, \tau)}\left|z^{\prime}(\tau)\right| \\
& -\frac{1}{2 \pi} J_{0}(k r(t, \tau)) \frac{z^{\prime}(\tau) \cdot h^{\prime}(\tau)}{\left|z^{\prime}(\tau)\right|} \\
L_{k}^{\prime(1)}(t, \tau, z, h)= & -\frac{k}{2 \pi} J_{1}(k r(t, \tau)) \frac{h^{\prime}(\tau)^{\perp} \cdot(z(t)-z(\tau))+z^{\prime}(\tau)^{\perp} \cdot(h(t)-h(\tau))}{r(t, \tau)} \\
& +\left\{-\frac{k^{2}}{2 \pi} J_{0}(k r(t, \tau))+\frac{k}{\pi} \frac{J_{1}(k r(t, \tau))}{r(t, \tau)}\right\} \times \\
& \times \frac{(z(t)-z(\tau)) \cdot(h(t)-h(\tau))}{[r(t, \tau)]^{2}} z^{\prime}(\tau)^{\perp} \cdot(z(t)-z(\tau)) \\
L_{0}^{\prime(1)}(t, \tau, z, h)= & 0 .
\end{aligned}
$$

Für die Diagonalterme erhält man unter anderem wegen $J_{0}(0)=1, J_{1}(0)=0$, und Taylorentwicklung die Ausdrücke

$$
\begin{aligned}
& M_{k}^{\prime(1)}(t, t, z, h)=-\frac{1}{2 \pi} \frac{z^{\prime}(t) \cdot h^{\prime}(t)}{\left|z^{\prime}(t)\right|}, \\
& M_{k}^{\prime(2)}(t, t, z, h)=\left(\frac{i}{2}-\frac{1}{\pi}\left\{1+C_{E}+\ln \left(\frac{k}{2}\left|z^{\prime}(t)\right|\right)\right\}\right) \frac{z^{\prime}(t) \cdot h^{\prime}(t)}{\left|z^{\prime}(t)\right|} \\
& L_{k}^{\prime(1)}(t, t, z, h)=0,
\end{aligned}
$$




$$
\begin{aligned}
L_{k}^{\prime(2)}(t, t, z, h) & =\frac{1}{2 \pi} \frac{h^{\prime}(t)^{\perp} \cdot z^{\prime \prime}(t)+z^{\prime}(t)^{\perp} \cdot h^{\prime \prime}(t)}{\left|z^{\prime}(t)\right|^{2}}-\frac{1}{\pi} \frac{z^{\prime}(t) \cdot h^{\prime}(t) z^{\prime}(t)^{\perp} \cdot z^{\prime \prime}(t)}{\left|z^{\prime}(t)\right|^{4}} \\
L_{0}^{\prime(1)}(t, t, z, h) & =0 \\
L_{0}^{\prime(2)}(t, t, z, h) & =L_{k}^{\prime(2)}(t, t, z, h) .
\end{aligned}
$$

Für eine feste Einfallsrichtung $d$ und ein $h \in Q_{N}$ wird mit der an den diskreten Stellen $z\left(s_{j}^{(n)}\right), j=0, \ldots, 2 n-1$, ermittelten Dichte

$$
\varphi=\left(I+A_{z}\right)^{-1} R_{z} u^{i} \in C\left(\partial D_{z}\right), \varphi_{j}^{(n)}=\varphi\left(z\left(s_{j}^{(n)}\right)\right)
$$

die rechte Seite in (5.18) ausgewertet durch

$$
\begin{aligned}
& 2 b(z(w(s))) \\
& \begin{aligned}
\approx & -2 i k e^{i k z(w(s)) \cdot d} d \cdot h(w(s)) \\
& -\frac{\pi}{n} \sum_{j=1}^{2 n-1} \alpha_{j}^{(n)}\left(L_{k}^{\prime(2)}-i \eta M_{k}^{\prime(2)}\right)\left(w(s), s_{j}^{(n)}, z, h\right)\left[\varphi_{j}^{(n)}-\varphi_{0}^{(n)}\right] \\
& -\frac{\pi}{n} \sum_{j=1}^{2 n-1} \alpha_{j}^{(n)}\left(L_{k}^{\prime(1)}-i \eta M_{k}^{\prime(1)}\right)\left(w(s), s_{j}^{(n)}, z, h\right) \times \\
& \left.-\varphi_{0}^{(n)} \frac{\pi}{n} \sum_{j=1}^{2 n-1} \alpha_{j}^{(n)}\left(L_{k}^{\prime(2)}-L_{0}^{\prime(2)}-i \eta M_{k}^{\prime(2)}\right)\left(w(s), s_{j}^{(n)}, z, h\right)+\frac{n}{\pi} R_{j}^{(n)}(s)\right]\left[\varphi_{j}^{(n)}-\varphi_{0}^{(n)}\right] \\
& -\varphi_{0}^{(n)} \frac{\pi}{n} \sum_{j=1}^{2 n-1} \alpha_{j}^{(n)}\left(L_{k}^{\prime(1)}-L_{0}^{\prime(1)}-i \eta M_{k}^{\prime(1)}\right)\left(w(s), s_{j}^{(n)}, z, h\right)\left[T\left(s, t_{j}^{(n)}\right)+\frac{n}{\pi} R_{j}^{(n)}(s)\right] .
\end{aligned}
\end{aligned}
$$

Dabei sind die Funktionen $T$ und $R_{j}^{(n)}$ durch (3.18) und (3.16) gegeben und die auftretenden Kerne in (6.9) - (6.20) erklärt. 


\subsection{Rekonstruktionen mit glatten Kurven}

Zunächst wird als Approximationsraum für die radialen Anteile der Raum der trigonometrischen Polynome

$\hat{Q}_{N}=T_{\tilde{N}}=\left\{\varphi \in C_{2 \pi} \mid \varphi(t)=\sum_{j=0}^{\tilde{N}} c_{j} \cos (j t)+\sum_{j=1}^{\tilde{N}} s_{j} \sin (j t), c_{0}, \ldots, c_{\tilde{N}}, s_{1}, \ldots, s_{\tilde{N}} \in \mathbb{R}\right\}$

mit $N=2 \tilde{N}+1$ verwendet.

In diesem Abschnitt wird versucht, die nachfolgenden Kurven zu rekonstruieren

$$
\begin{aligned}
\text { Erdnuss: } z(t) & =\sqrt{\cos ^{2}(t)+0.25 \sin ^{2}(t)}\left(\begin{array}{c}
\cos (t) \\
\sin (t)
\end{array}\right), \\
\text { Drachen: } z(t) & =\left(\begin{array}{c}
\cos (t)+0.65(\cos (2 t)-1) \\
1.5 \sin (t)
\end{array}\right), \\
\text { Tropfen: } z(t) & =\left(\begin{array}{c}
-2 / \sqrt{3} \sin (0.5 t)+0.5 \\
\sin (t)
\end{array}\right), \\
\text { Schmetterling: } z(t) & =\left(\begin{array}{c}
2 / 3 \sin (1.5 t)+0.3 \\
\sin (t)
\end{array}\right) .
\end{aligned}
$$

Die synthetischen Fernfelddaten $u_{\infty}\left(\hat{x}_{i}, d_{l}\right), 1 \leq i \leq M, 1 \leq l \leq D$, wurden mit den Parametern $k=1, \eta=1, n=64$ und der polynomialen Substitution $w_{m}$ (3.9) nach Korobov mit Substitutionsgrad $m=4$ (beim Tropfen und Schmetterling) beziehungsweise $w(t)=t$ (für die Erdnuss und den Drachen) erzeugt.

Für den inversen Löser wurde mit der Substitution (3.11) nach Kreß mit Grad $m=4$, mit exakter Wellenzahl $k=1$ und Kopplungsparameter $\eta=1.2$ gerechnet. Alle übrigen Parameter, wie die Anzahl der Stützstellen 2n, die Dimension $N$ des Ansatzraumes, unterschiedliche Anzahl von Beobachtungs- und Einfallsrichtungen $M$ bzw. $D$, der Regularisierungsparameter $\alpha_{p}$ im $p$-ten Iterationsschritt, die verwendete Sobolevnorm $\|\cdot\|_{H^{s}}$ und zugehörige Anzahl $K$ von Stützstellen sind den Legenden zu entnehmen.

Als Startkurve $z_{0}$ diente jeweils der Einheitskreis

$$
z_{0}(t)=\left(\begin{array}{c}
\cos (t) \\
\sin (t)
\end{array}\right), 0 \leq t \leq 2 \pi .
$$

Es wurden jeweils 30 Newton-Schritte bei der Erdnuss, 70 beim Drachen, 40 beim Tropfen und 50 Iterationen beim Schmetterling durchgeführt, wobei jedoch zur Darstellung die Kurve $z_{p_{\text {end }}}$ zum kleinsten Residuum verwendet wurde. 
Rekonstruktion der Erdnuss mit einer Welle

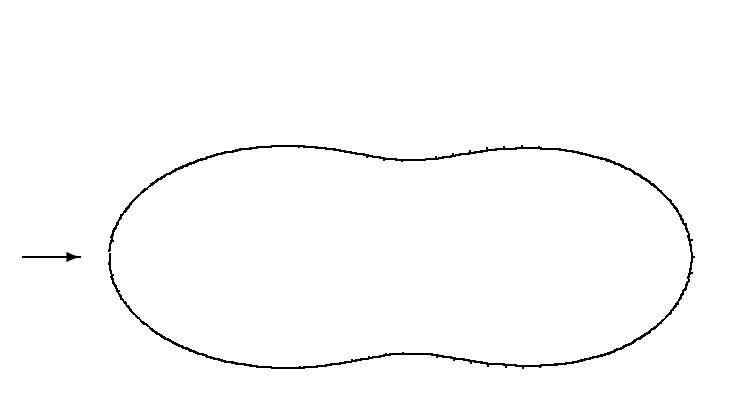

$$
\begin{aligned}
p_{\text {end }} & =30, \\
n & =48, \\
N & =11, \\
M & =64, \\
D & =1, \\
\alpha_{p} & =1.0 \cdot 0.6^{p}, \\
\text { Res } & =1.3 \cdot 10^{-7}, \\
s & =1, \\
K & =128 .
\end{aligned}
$$

Rekonstruktion der Erdnuss mit 4 Wellen

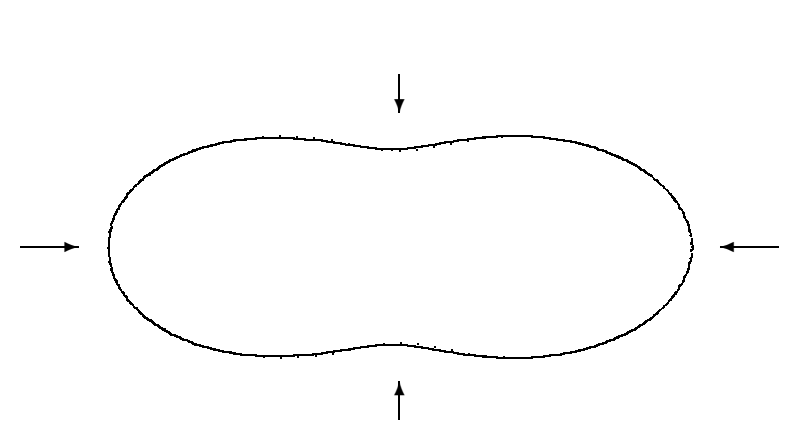

$$
\begin{aligned}
p_{\text {end }} & =26, \\
n & =48, \\
N & =11, \\
M & =64, \\
D & =4, \\
\alpha_{p} & =1.0 \cdot 0.6^{p}, \\
\text { Res } & =4.9 \cdot 10^{-7}, \\
s & =1, \\
K & =128 .
\end{aligned}
$$

Rek. der Erdnuss mit kleinerem $\mathrm{n}$ und mehr Ansatzfunktionen

$$
\begin{aligned}
p_{\text {end }} & =29, \\
n & =24, \\
N & =15, \\
M & =64, \\
D & =4, \\
\alpha_{p} & =1.0 \cdot 0.6^{p}, \\
\text { Res } & =4.9 \cdot 10^{-7}, \\
s & =2, \\
K & =128 .
\end{aligned}
$$


Rekonstruktion des Drachens mit einer Welle

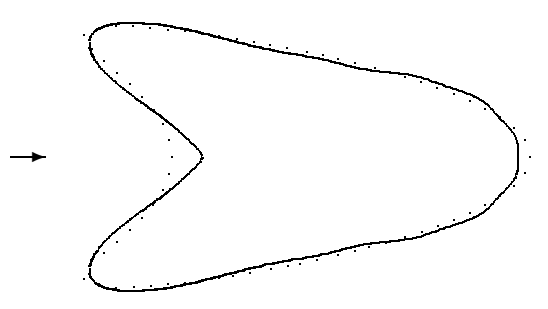

$$
\begin{aligned}
p_{\text {end }} & =70, \\
n & =36, \\
N & =25, \\
M & =64, \\
D & =1, \\
\alpha_{p} & =10.0 \cdot 0.9^{p}, \\
R e s & =1.7 \cdot 10^{-5} \\
s & =1 \\
K & =128 .
\end{aligned}
$$

$$
\begin{aligned}
p_{\text {end }} & =60, \\
n & =36, \\
N & =25, \\
M & =64, \\
D & =4, \\
\alpha_{p} & =2.0 \cdot 0.8^{p}, \\
R e s & =1.3 \cdot 10^{-8} \\
s & =1, \\
K & =256 .
\end{aligned}
$$

Rek. des Drachens mit s=3 und weniger Ansatzfkt.

$$
\begin{aligned}
p_{\text {end }} & =70, \\
n & =24, \\
N & =15, \\
M & =64, \\
D & =4, \\
\alpha_{p} & =10.0 \cdot 0.9^{p}, \\
R e s & =3.9 \cdot 10^{-3}, \\
s & =3, \\
K & =256 .
\end{aligned}
$$


Rekonstruktion des Tropfens mit einer Welle

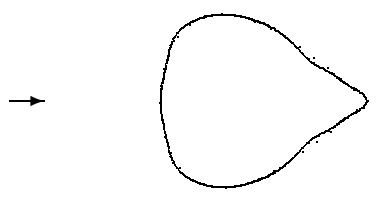

Rekonstruktion des Tropfens mit 4 Wellen

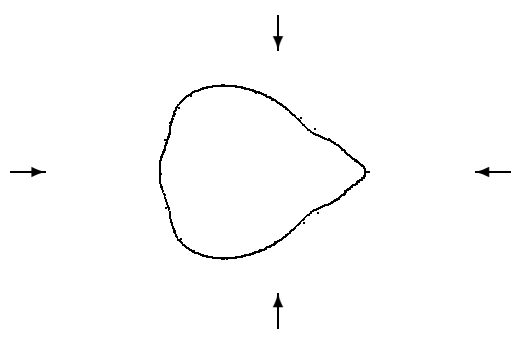

Rek. des Tropfens mit weniger Ansatzfunktionen

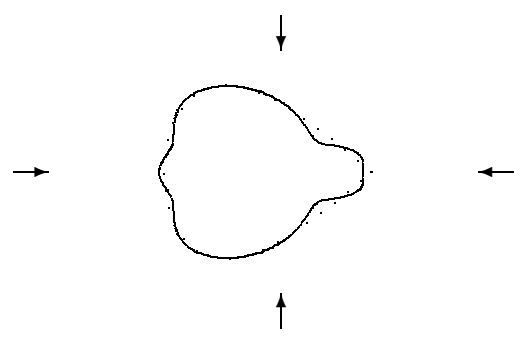

$$
\begin{aligned}
p_{\text {end }} & =40, \\
n & =36, \\
N & =15, \\
M & =64, \\
D & =1, \\
\alpha_{p} & =2.0 \cdot 0.8^{p}, \\
R e s & =9.5 \cdot 10^{-8}, \\
s & =1, \\
K & =128 .
\end{aligned}
$$

$$
\begin{aligned}
p_{\text {end }} & =40, \\
n & =36, \\
N & =15, \\
M & =64, \\
D & =4, \\
\alpha_{p} & =2.0 \cdot 0.8^{p}, \\
R e s & =4.0 \cdot 10^{-8}, \\
s & =1, \\
K & =128 .
\end{aligned}
$$

$$
\begin{aligned}
p_{\text {end }} & =40, \\
n & =24, \\
N & =11, \\
M & =64, \\
D & =4, \\
\alpha_{p} & =2.0 \cdot 0.8^{p}, \\
R e s & =6.2 \cdot 10^{-8}, \\
s & =1, \\
K & =256 .
\end{aligned}
$$


Rekonstruktion des Schmetterlings mit einer Welle

$$
\begin{aligned}
p_{\text {end }} & =50, \\
n & =48, \\
N & =17, \\
M & =64, \\
D & =1, \\
\alpha_{p} & =5.0 \cdot 0.85^{p}, \\
R e s & =1.1 \cdot 10^{-5}, \\
s & =1, \\
K & =128 .
\end{aligned}
$$$$
\rightarrow
$$

Rekonstruktion des Schmetterlings mit 4 Wellen

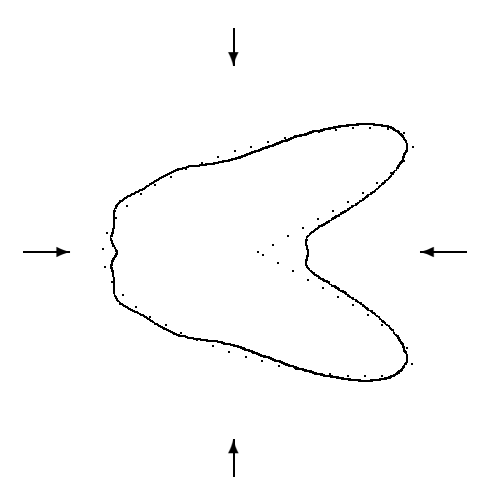

$$
\begin{aligned}
p_{\text {end }} & =50, \\
n & =48, \\
N & =21, \\
M & =64, \\
D & =4, \\
\alpha_{p} & =5.0 \cdot 0.85^{p}, \\
R e s & =1.6 \cdot 10^{-6}, \\
s & =1, \\
K & =128 .
\end{aligned}
$$

Rek. des Schmetterlings mit weniger Ansatzfunktionen

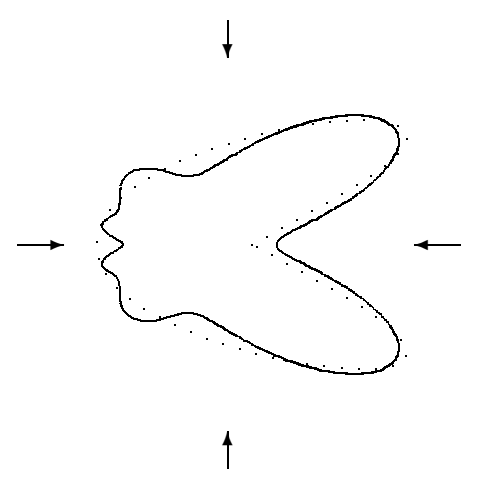

$$
\begin{aligned}
p_{\text {end }} & =50, \\
n & =24, \\
N & =15, \\
M & =64, \\
D & =4, \\
\alpha_{p} & =5.0 \cdot 0.85^{p}, \\
R e s & =3.3 \cdot 10^{-6}, \\
s & =1, \\
K & =128 .
\end{aligned}
$$


Bei den numerischen Experimenten wurden folgende Beobachtungen gemacht:

Die konvexen Gebiete lassen sich stets besser rekonstruieren, als nicht-konvexe Gebiete. Das Verfahren liefert im ersten Fall, sowohl bei glatten Rändern, als auch bei Gebieten mit Ecken, bei geringer Anzahl von Basisfunktionen und wenigen Newton-Schritten gute Rekonstruktionen.

Um gute Approximationen an nicht-konvexe Gebiete zu erhalten, ist eine höhere Anzahl an Basisfunktionen und Newton-Schritten erforderlich. Zusätzlich muss das Verfahren mit einem hohen Regularisierungsparameter gestartet werden, der im Laufe des Verfahrens nicht zu schnell abfallen darf.

Die Experimente, bei denen im Strafterm $\|h\|_{H^{s}}$ der Parameter $s=3$ verwendet wurde, lieferten teilweise unzulässige Ränder, das heißt die ermittelten Parametrisierungen waren auf $[0,2 \pi)$ nicht injektiv.

\subsection{Rekonstruktionen mit nicht-glatten Kurven}

Die Funktionen $r_{1}, r_{2}$, mit

$$
\begin{aligned}
& r_{1}(t)=t^{2}(2 \pi-t), 0 \leq t \leq 2 \pi \\
& r_{2}(t)=t(2 \pi-t)^{2}, 0 \leq t \leq 2 \pi
\end{aligned}
$$

besitzen die Eigenschaften

$$
r_{1}(0)=r_{1}(2 \pi)=0=r_{2}(0)=r_{2}(2 \pi)
$$

und

$$
r_{1}^{\prime}(0)=r_{2}^{\prime}(2 \pi)=0, r_{1}^{\prime}(2 \pi)=-4 \pi^{2}, r_{2}^{\prime}(0)=4 \pi^{2}
$$

Demnach sind

$$
q_{j}(t)=r_{j}(t)(\cos t, \sin t)^{t}, 0 \leq t \leq 2 \pi, j=1,2,
$$

periodisch mit Periode $2 \pi$, auf $[0,2 \pi]$ beliebig oft differenzierbar und die Gebiete mit durch $q_{1}, q_{2}$ parametrisierten Rändern besitzen Ecken.

Für den Ansatzraum werden nun die Terme $\sin (\tilde{N} t)$ und $\cos (\tilde{N} t)$ weggelassen und dafür $r_{1}, r_{2}$ hinzugefügt, das heißt

$$
\hat{Q}_{N}=\left\{\varphi \in C_{2 \pi} \mid \varphi(t)=\sum_{j=0}^{\tilde{N}-1} c_{j} \cos (j t)+\sum_{j=1}^{\tilde{N}-1} s_{j} \sin (j t)+c_{\tilde{N}} r_{1}(t)+s_{\tilde{N}} r_{2}(t)\right\}
$$


$\operatorname{mit} N=2 \tilde{N}+1$.

In diesem Abschnitt wird versucht, die nachfolgenden Kurven zu rekonstruieren

$$
\begin{aligned}
\text { Tropfen: } z(t) & =\left(\begin{array}{c}
-2 / \sqrt{3} \sin (0.5 t)+0.5 \\
\sin (t)
\end{array}\right), \\
\text { Schmetterling: } z(t) & =\left(\begin{array}{c}
2 / 3 \sin (1.5 t)+0.3 \\
\sin (t)
\end{array}\right) .
\end{aligned}
$$

Die synthetischen Fernfelddaten $u_{\infty}\left(\hat{x}_{i}, d_{l}\right), 1 \leq i \leq M, 1 \leq l \leq D$, wurden diesmal mit den Parametern $k=2, \eta=2, n=64$ und der polynomialen Substitution $w_{m}$ (3.9) nach Korobov mit Substitutionsgrad $m=4$ erzeugt.

Für den inversen Löser wurde mit exakter Wellenzahl $k$, dem Kopplungsparameter $\eta=$ 2.5, der Substitution (3.11) von Kreß mit Grad $m=4$ und dem Einheitskreis als Startkurve gerechnet.

Rekonstruktion des Tropfens mit einer Welle

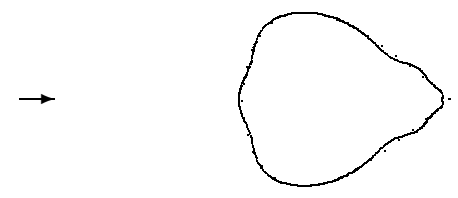

Rekonstruktion des Tropfens mit 4 Wellen

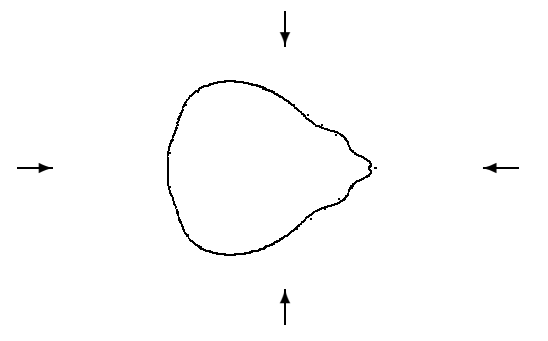

$$
\begin{aligned}
p_{\text {end }} & =40, \\
n & =36, \\
N & =17, \\
M & =64, \\
D & =1, \\
\alpha_{p} & =2.0 \cdot 0.8^{p}, \\
R e s & =1.4 \cdot 10^{-7}, \\
s & =1, \\
K & =128 .
\end{aligned}
$$

$$
\begin{aligned}
p_{\text {end }} & =40, \\
n & =36, \\
N & =17 \\
M & =64, \\
D & =4, \\
\alpha_{p} & =2.0 \cdot 0.8^{p}, \\
R e s & =2.3 \cdot 10^{-7} \\
s & =1, \\
K & =128 .
\end{aligned}
$$


Rek. des Tropfens mit mehr Ansatzfunktionen

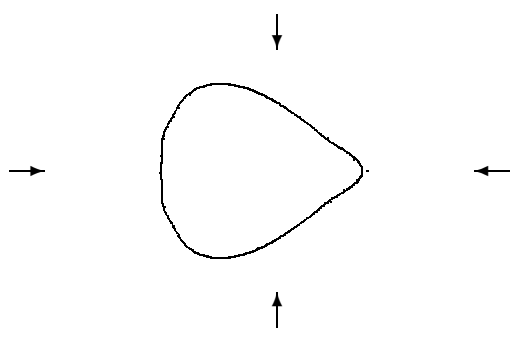

Rekonstruktion des Schmetterlings mit einer Welle

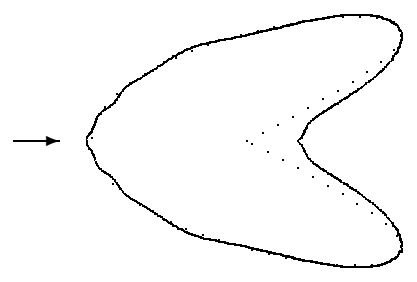

Rekonstruktion des Schmetterlings mit 4 Wellen

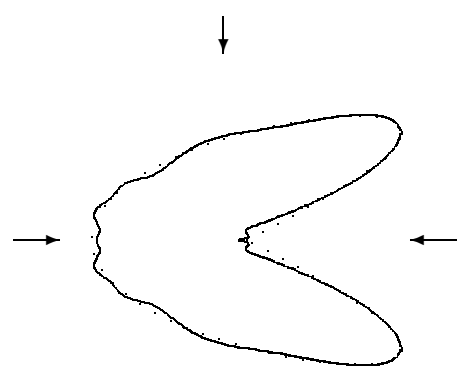

$$
\uparrow
$$

$$
\begin{aligned}
p_{\text {end }} & =40, \\
n & =48, \\
N & =25, \\
M & =64, \\
D & =4, \\
\alpha_{p} & =3.0 \cdot 0.8^{p}, \\
R e s & =8.1 \cdot 10^{-9}, \\
s & =1, \\
K & =128 .
\end{aligned}
$$

$$
\begin{aligned}
p_{\text {end }} & =50, \\
n & =48, \\
N & =25, \\
M & =64, \\
D & =1 \\
\alpha_{p} & =5.0 \cdot 0.85^{p}, \\
R e s & =1.2 \cdot 10^{-5}, \\
s & =1 \\
K & =128 .
\end{aligned}
$$

$$
\begin{aligned}
p_{\text {end }} & =50, \\
n & =48, \\
N & =25, \\
M & =64, \\
D & =4, \\
\alpha_{p} & =5.0 \cdot 0.85^{p}, \\
R e s & =8.8 \cdot 10^{-7}, \\
s & =1, \\
K & =128 .
\end{aligned}
$$


Rek. des Schmetterlings mit weniger Ansatzfkt.

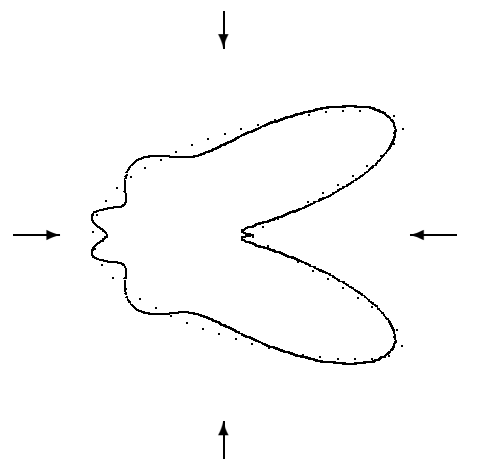

$$
\begin{aligned}
p_{\text {end }} & =50, \\
n & =36, \\
N & =17, \\
M & =64, \\
D & =4, \\
\alpha_{p} & =10.0 \cdot 0.9^{p}, \\
R e s & =2.6 \cdot 10^{-4}, \\
s & =1, \\
K & =128 .
\end{aligned}
$$

An der letzten Darstellung (und weiteren, hier nicht angeführten Beispielen) wird deutlich, dass bei Rekonstruktionen mit einer zu geringen Anzahl von Ansatzfunktionen, die Größe des Eckenwinkels nicht mehr rekonstruiert wird.

Ein Vergleich der Ergebnisse aus den Abschnitten 6.4 und 6.5 lässt keine eindeutige Schlussfolgerung zu. Während sich beim Tropfen die Hinzunahme nicht-glatter Ansatzfunktionen negativ auf das Residuum auswirkt, verhält es sich beim Schmetterling genau umgekehrt. Theoretisch könnte man auf ein anderes Qualitätsmerkmal statt dem Residuum ausweichen, etwa dem Vergleich der Radialanteile von exakter und numerischer Lösung in der $C^{2}$-Norm. Auf der anderen Seite erscheint dieser Ausweg wenig sinnvoll, denn im Allgemeinen ist der exakte Rand unbekannt und ist erst zu rekonstruieren.

\subsection{Eingeschränkter Messbereich}

In diesem Abschnitt wurde versucht, mit den gleichen Ansatzfunktionen wie im Abschnitt 6.5 den Tropfen und Schmetterling zu rekonstruieren, wobei die Messpunkte $\hat{x}_{i}$, an denen das Fernfeld gemessen wird, nicht gleichmäßig auf dem Einheitskreis verteilt sind, sondern nur aus einem eingeschränkten Winkelbereich stammen.

Für die Erzeugung der Messdaten und den Parametern im inversen Löser gilt das in Abschnitt 6.5 gesagte. In den Darstellungen wurde zusätzlich der „beleuchtete Bereich“ gepunktet aufgenommen. Für jedes Objekt wurde mit einer einzigen einfallenden Welle und einem Halbkreis als Messbereich gearbeitet. 
Tropfen: Eckenbereich im Schatten

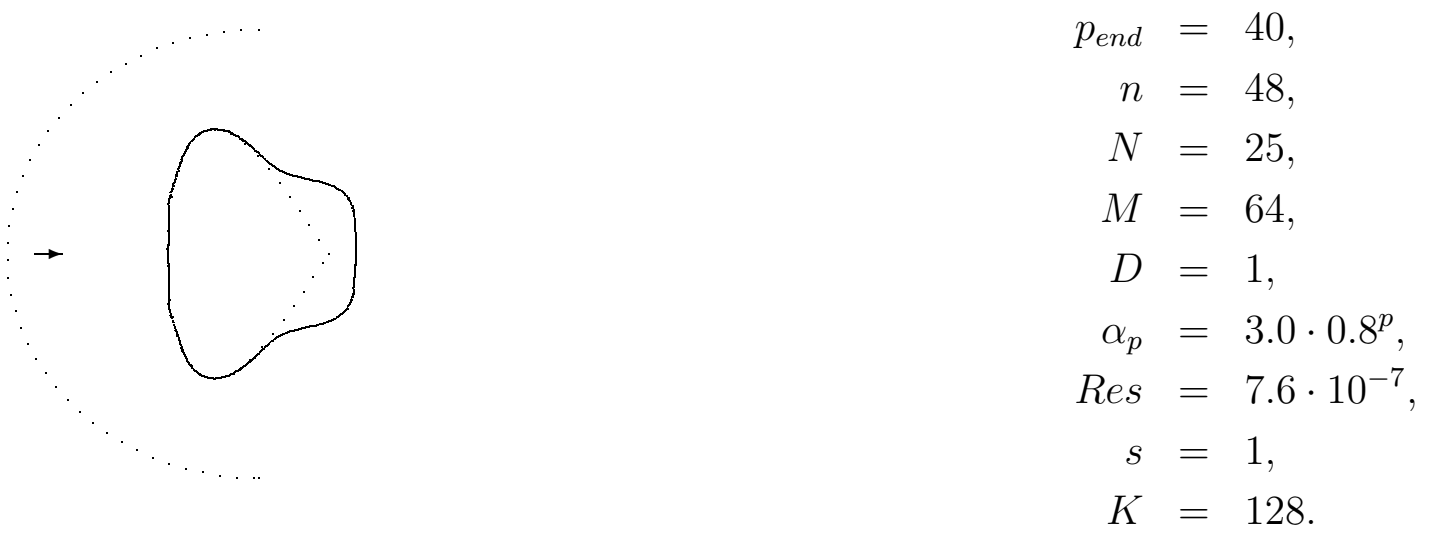

Tropfen: Eckenbereich beleuchtet

$$
\begin{aligned}
p_{\text {end }} & =40, \\
n & =48, \\
N & =25, \\
M & =64, \\
D & =1, \\
\alpha_{p} & =3.0 \cdot 0.8^{p}, \\
\text { Res } & =1.5 \cdot 10^{-6}, \\
s & =1, \\
K & =128 .
\end{aligned}
$$

Tropfen: Messungen von unten

$$
\begin{aligned}
p_{\text {end }} & =40, \\
n & =48, \\
N & =25, \\
M & =64, \\
D & =1, \\
\alpha_{p} & =3.0 \cdot 0.8^{p}, \\
\text { Res } & =3.8 \cdot 10^{-6}, \\
s & =1, \\
K & =128 .
\end{aligned}
$$


Schmetterling: Eckenbereich im Schatten

$$
\begin{aligned}
p_{\text {end }} & =50, \\
n & =48, \\
N & =25, \\
M & =64, \\
D & =1, \\
\alpha_{p} & =5.0 \cdot 0.85^{p}, \\
R e s & =3.1 \cdot 10^{-6}, \\
s & =1, \\
K & =128 .
\end{aligned}
$$

Schmetterling: Eckenbereich beleuchtet

$$
\begin{aligned}
p_{\text {end }} & =50, \\
n & =48, \\
N & =25, \\
M & =64, \\
D & =1, \\
\alpha_{p} & =5.0 \cdot 0.85^{p}, \\
R e s & =9.2 \cdot 10^{-6}, \\
s & =1, \\
K & =128 .
\end{aligned}
$$

Schmetterling: Messungen von unten

$$
\begin{aligned}
p_{\text {end }} & =50, \\
n & =48, \\
N & =25, \\
M & =64, \\
D & =1, \\
\alpha_{p} & =5.0 \cdot 0.85^{p}, \\
R e s & =2.4 \cdot 10^{-6}, \\
s & =1, \\
K & =128 .
\end{aligned}
$$


Aus den zahlreichen numerischen Experimenten lässt sich feststellen, dass die Rekonstruktionen bei eingeschränktem Messbereich deutlich schlechter sind als bei vollem Datensatz. Auch durch Hinzunahme weiterer Basisfunktionen und höhere Anzahl von NewtonSchritten wurde der nicht „beleuchtete Teil“ des Gebietes schlecht rekonstruiert. In den Beispielen, bei denen der Eckenbereich beleuchtet wurde, ergaben sich bessere Ergebnisse. Die mit Abstand besten Rekonstruktionen wurden bei „Beleuchtung“ im unteren (oder oberen) Bereich erzielt. Am Residuum, dem eigentlich einzigen objektiven Kriterium (denn der Rand ist ja unbekannt), ist diese Beobachtung nicht abzulesen.

Wiederum ist festzustellen, dass sich konvexe Gebiete mit weniger Aufwand (kleinere $\left.N, p_{\text {end }}, n\right)$ rekonstruieren lassen, als nicht-konvexe Gebiete. 


\section{Kapitel 7}

\section{Ein spezielles \\ Impedanzrandwertproblem}

In diesem Kapitel wird ein weiteres Randwertproblem betrachtet, bei dem in natürlicher Weise Ecken auftreten. Nach Vergraben eines metallischen Gegenstandes, von dem ein Teil weiterhin aus dem Boden herausragt, werden Korrosionseffekte zu Veränderungen der Oberfläche sorgen. Man ist bestrebt, die nicht einsichtbare Oberfläche aufgrund von (Spannungs-) Messungen zu ermitteln, ohne den Gegenstand aus der Erde auszugraben. Zunächst wird jedoch an dieser Stelle das direkte Problem behandelt.

Nach der mathematischen Formulierung des resultierenden Randwertproblems erfolgt im Abschnitt 7.2 der Nachweis der Existenz und Eindeutigkeit. Für die numerische Umsetzung wird der hypersinguläre Operator $T_{0}$ benötigt, dessen Darstellung im dritten Teil notiert wird. Abschließend sind im Abschnitt 7.4 für verschiedene Objekte numerische Beispiele angeführt.

\subsection{Formulierung des Impedanzrandwertproblems}

Gegeben sei ein beschränktes, leitfähiges Medium $D \subset \mathbb{R}^{2}$, dessen Leitfähigkeit im gesamten Gebiet $D$ konstant ist. Der Rand $\partial D$ von $D$ setze sich aus zwei zusammenhängenden, $C^{3}$-glatten Randstücken $\Gamma, \gamma$ zusammen, sodass gilt

$$
\partial D=\Gamma \cup \gamma, \Gamma \cap \gamma=\left\{x^{(0)}, x^{(1)}\right\}
$$

Desweiteren setze $\Gamma_{0}:=\Gamma \backslash\left\{x^{(0)}, x^{(1)}\right\}$ und $\gamma_{0}:=\gamma \backslash\left\{x^{(0)}, x^{(1)}\right\}$. 


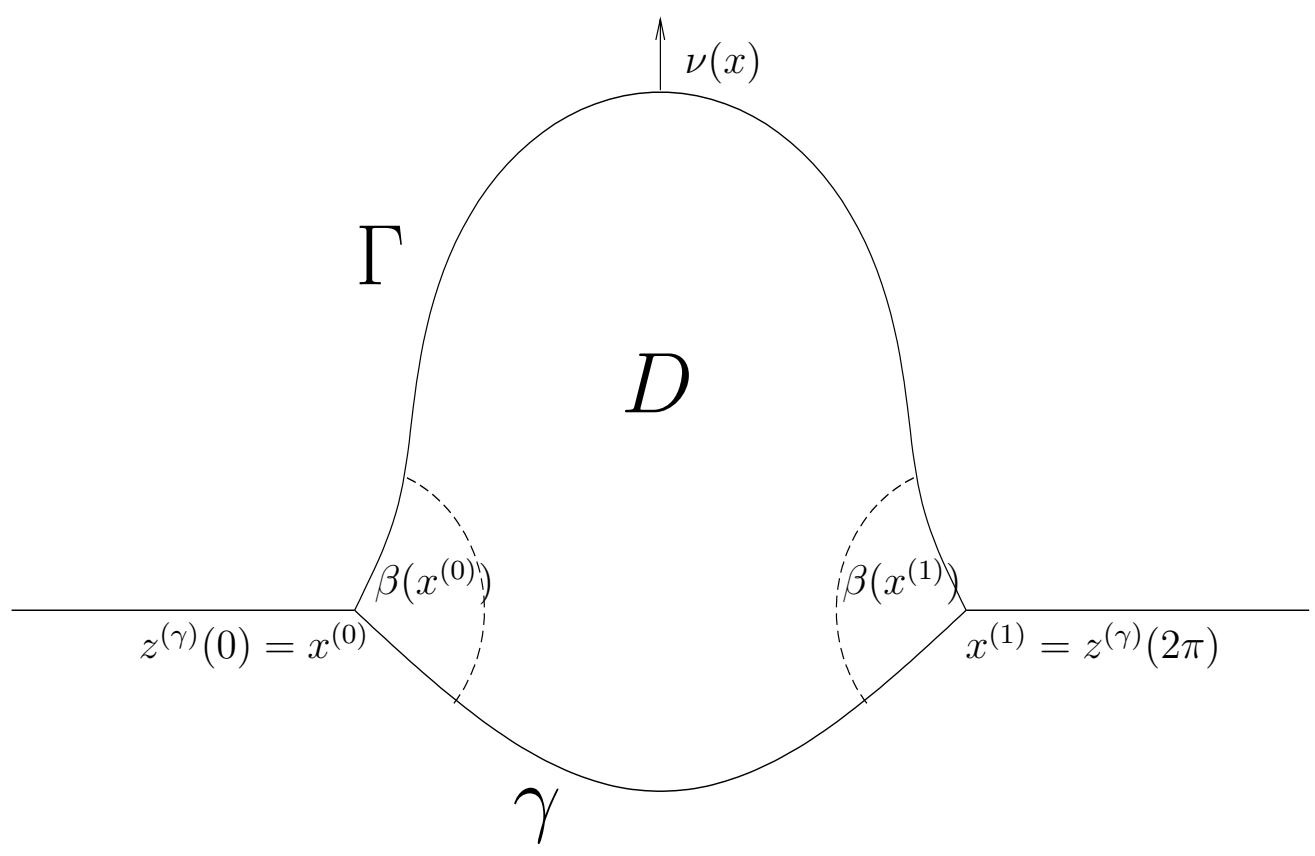

Darstellung zum Impedanzproblem

Ohne Beeinträchtigung der Allgemeinheit wird von den Ecken $x^{(0)}, x^{(1)}$ vorausgesetzt, dass deren zweite Koordinaten Null sind. Desweiteren gelten einschränkend für die Parametrisierungen $z^{(\Gamma)}, z^{(\gamma)}$ der Randstücke $\Gamma, \gamma$ nachfolgende Beziehungen:

$$
\begin{aligned}
z^{(\gamma)}(0)=z^{(\Gamma)}(4 \pi) & =x^{(0)}, \\
z^{(\gamma)}(2 \pi)=z^{(\Gamma)}(2 \pi) & =x^{(1)}, \\
z_{2}^{(\Gamma)}(t) & >0,2 \pi<t<4 \pi, \\
z_{2}^{(\gamma)}(t) & <0,0<t<2 \pi .
\end{aligned}
$$

\section{Definition 7.1 (Inneres Dirichletproblem)}

Gegeben ist $f \in C(\partial D)$. Gesucht wird eine Funktion $u \in C^{2}(D) \cap C(\bar{D})$ als Lösung der Laplacegleichung

$$
\triangle u=0 \text { in } D,
$$


die die Randbedingung

$$
u=f \quad \text { auf } \partial D
$$

erfüllt.

Bei der Modellierung des eingangs von Kapitel 7 geschilderten Problems repräsentiert $u$ eine Spannung. Weil auf dem korrodierten Teil des Randes kein Strom fließt, die Spannung demzufolge Null ist, erhält das direkte Problem die nachfolgende Form.

\section{Definition 7.2 (Direktes Problem)}

Gegeben sind $D$ und $f \in C(\partial D)$. Desweiteren erfülle $f$ die Bedingungen $\left.f\right|_{\Gamma} \in C^{1, \alpha}(\Gamma)$ und $\left.f\right|_{\gamma}=0$. Gesucht werden die Neumannrandwerte

$$
\left.\frac{\partial u}{\partial \nu}\right|_{\Gamma_{0}}
$$

der nach Abschnitt 7.9 eindeutig bestimmten Lösung u von (7.1) - (7.9).

\section{Definition 7.3 (Inverses Problem)}

Für festes $\Gamma$ ist der Dirichlet- nach Neumannoperator gegeben, das heißt für alle $f$ gemäß Definition 7. g sind die Paare $\left(\left.f\right|_{\Gamma},\left.\frac{\partial u}{\partial \nu}\right|_{\Gamma_{0}}\right)$ bekannt. Gesucht wird der Rand $\gamma$.

\subsection{Existenz und Eindeutigkeit beim direkten Impedanzrandwertproblem}

Satz 7.4 Das Randwertproblem (7.1) - (7.9) besitzt höchstens eine Lösung.

Beweis: Das Maximum-Minimum Prinzip für harmonische Funktionen erfordert keine Randglätte.

Satz 7.5 Das Gebiet D erfülle die angegebenen Voraussetzungen. Sei $f \in C(\partial D)$. Dann gilt: Für jede Lösung $\varphi \in C(\partial D)$ der Integralgleichung

$$
-2 f(x)=\frac{2 \pi-\beta(x)}{\pi} \varphi(x)-2 \int_{\partial D} \frac{\partial \Phi_{0}(x, y)}{\partial \nu(y)} \varphi(y) d s(y), x \in \partial D,
$$


wird durch das Doppelschichtpotenzial

$$
u(x)=\int_{\partial D} \frac{\partial \Phi_{0}(x, y)}{\partial \nu(y)} \varphi(y) d s(y), x \in D,
$$

eine Lösung des inneren Dirichletproblems (7.1) - (7.2) gegeben.

Beweis: Das Doppelschichtpotenzial ist harmonisch in $D$ und lässt sich nach Satz 4.12 von $D$ nach $\bar{D}$ stetig fortsetzen mit den Randwerten

$$
2 u_{-}(x)=\frac{\beta(x)-2 \pi}{\pi} \varphi(x)+2 \int_{\partial D} \frac{\partial \Phi_{0}(x, y)}{\partial \nu(y)} \varphi(y) d s(y)=2 f(x), x \in \partial D .
$$

Satz 7.6 Die Integralgleichung (7.3) hat für jedes $f \in C(\partial D)$ genau eine Lösung $\varphi \in C(\partial D)$.

Beweis: Die Injektivität von (7.3) zeigt man wie in [35, Theorem 6.20] unter anderem mit der Eindeutigkeit des äußeren Neumannproblems. Für den Nachweis der Surjektivität ist wie im Beweis von Satz 4.14 eine Aufspaltung in einen beschränkt invertierbaren und einen kompakten Operator hinreichend. Es kann wiederum auf [2ָ, Sätze 3.12 - 3.13] verwiesen werden, wobei wegen der fehlenden logarithmischen Singularität (setze $\eta=0$ ) und dem gleichen singulären Verhalten von $\Phi_{k}$ und $\Phi_{0}$ sich die Darstellung vereinfacht. Für den Spezialfall, bei dem der Rand in der Nähe der Ecken geradlinig verläuft, ist der vereinfachte Beweis in [35, Section 6.5] nachzulesen.

Auf die Randintegralgleichung (7.3) lässt sich das Nyström-Verfahren nicht direkt anwenden, denn die Gleichung hat nicht die Form (3.1) aus Kapitel 3. Daher wird eine Modifizierung erforderlich. Dazu seien $u_{j}, j=0,1$, auf $\mathbb{R}^{2}$ harmonische Funktionen mit $u_{j}\left(x^{(k)}\right)=\delta_{j k}$. Schreibt man $x^{(j)}=\left(x_{1}^{(j)}, x_{2}^{(j)}\right)$, so erfüllen die bezüglich $x_{1}$ linearen Funktionen

$$
\begin{aligned}
& u_{0}(x)=\frac{x_{1}-x_{1}^{(1)}}{x_{1}^{(0)}-x_{1}^{(1)}}, \\
& u_{1}(x)=\frac{x_{1}-x_{1}^{(0)}}{x_{1}^{(1)}-x_{1}^{(0)}},
\end{aligned}
$$


die Forderungen. Mit Hilfe des Darstellungssatzes

$$
\int_{\partial D}\left\{\Phi_{0}(x, y) \frac{\partial u_{l}}{\partial \nu}(y)-\frac{\partial \Phi_{0}(x, y)}{\partial \nu(y)} u_{l}(y)\right\} d s(y)=\frac{\beta(x)}{2 \pi} u_{l}(x), x \in \partial D,
$$

und der Identität

$$
\left(1-\frac{\beta(x)}{\pi}\right) \varphi(x)+\sum_{l=0}^{1} \frac{\beta(x)}{\pi} \varphi\left(x^{(l)}\right) u_{l}(x)=\sum_{l=0}^{1} \varphi\left(x^{(l)}\right) u_{l}(x), x \in \partial D,
$$

wird die rechte Seite in (7.3) umgeformt zu

$$
\begin{aligned}
-2 f(x)= & \varphi(x)-\left(K_{0} \varphi\right)(x) \\
= & \frac{2 \pi-\beta(x)}{\pi} \varphi(x)-2 \int_{\partial D} \frac{\partial \Phi_{0}(x, y)}{\partial \nu(y)}\left\{\varphi(y)-\sum_{l=0}^{1} u_{l}(y) \varphi\left(x^{(l)}\right)\right\} d s(y) \\
& -2 \sum_{l=0}^{1} \varphi\left(x^{(l)}\right) \int_{\partial D} \frac{\partial \Phi_{0}(x, y)}{\partial \nu(y)} u_{l}(y) d s(y) \\
= & \varphi(x)-\left(\tilde{K}_{0} \varphi\right)(x)+\sum_{l=0}^{1} \varphi\left(x^{(l)}\right)\left\{u_{l}(x)-2 \int_{\partial D} \Phi_{0}(x, y) \frac{\partial u_{l}}{\partial \nu}(y) d s(y)\right\} .
\end{aligned}
$$

Dabei sind die Operatoren $K_{0}$ und $\tilde{K}_{0}$ gegeben durch

$$
\begin{aligned}
& \left(K_{0} \varphi\right)(x)=-\left(1-\frac{\beta(x)}{\pi}\right) \varphi(x)+2 \int_{\partial D} \frac{\partial \Phi_{0}(x, y)}{\partial \nu(y)} \varphi(y) d s(y), x \in \partial D, \varphi \in C(\partial D), \\
& \left(\tilde{K}_{0} \varphi\right)(x)=2 \int_{\partial D} \frac{\partial \Phi_{0}(x, y)}{\partial \nu(y)}\left\{\varphi(y)-\sum_{l=0}^{1} u_{l}(y) \varphi\left(x^{(l)}\right)\right\} d s(y), x \in \partial D, \varphi \in C(\partial D) .
\end{aligned}
$$

Insbesondere verschwindet bei $\tilde{K}_{0}$ die Dichte in den Ecken $x^{(0)}$ und $x^{(1)}$, sodass die Gleichung (7.4), abgesehen von geringen Modifizierungen, mit dem bereits in Kapitel 3 erklärten Nyström-Verfahren behandelt werden kann.

Die bereits aus Abschnitt 3.2 bekannten Substitutionen $w:[0,2 \pi] \rightarrow[0,2 \pi]$ werden zur Ermittlung der Näherungswerte

$$
\varphi_{j}^{(n)} \approx \varphi(z(w(j \pi / n))), j=0, \ldots, 4 n-1,
$$


durch

$$
w(t+2 \pi):=2 \pi+w(t), 0 \leq t \leq 2 \pi,
$$

auf $[0,4 \pi]$ fortgesetzt. Mit den Bezeichnungen

$$
\begin{aligned}
& t_{j}^{(n)}= \frac{j \pi}{n}, s_{j}^{(n)}=w\left(\frac{j \pi}{n}\right), \alpha_{j}^{(n)}=w^{\prime}\left(\frac{j \pi}{n}\right), f_{j}^{(n)}=-2 f\left(z\left(s_{j}^{(n)}\right)\right), j=0, \ldots, 4 n, \\
& u_{l, k}^{(0)}=u_{l}\left(z\left(s_{k}^{(n)}\right)\right), \\
& u_{l, k}^{(1)}=\frac{\partial u_{l}}{\partial \nu}\left(z\left(s_{k}^{(n)}\right)\right), l \in\{0,1\}, k=1, \ldots, 2 n-1,2 n+1, \ldots, 4 n-1,
\end{aligned}
$$

den Kernen

$$
\begin{aligned}
L_{0}(t, \tau) & =\frac{1}{\pi} \frac{z^{\prime}(\tau)^{\perp} \cdot(z(t)-z(\tau))}{|z(t)-z(\tau)|^{2}} \\
M_{0}(t, \tau) & =\frac{1}{\pi} \ln \frac{1}{|z(t)-z(\tau)|}\left|z^{\prime}(\tau)\right| \\
& =M_{0}^{(1)}(t, \tau) \ln \left(4 \sin ^{2} \frac{t-\tau}{2}\right)+M_{0}^{(2)}(t, \tau), \\
M_{0}^{(1)}(t, \tau) & =-\frac{1}{2 \pi}\left|z^{\prime}(\tau)\right| \\
M_{0}^{(2)}(t, \tau) & =M_{0}(t, \tau)-M_{0}^{(1)}(t, \tau) \ln \left(4 \sin ^{2} \frac{t-\tau}{2}\right),
\end{aligned}
$$

und den Diagonaltermen

$$
\begin{aligned}
L_{0}(t, t) & =\frac{1}{2 \pi} \frac{z^{\prime}(t)^{\perp} \cdot z^{\prime \prime}(t)}{\left|z^{\prime}(t)\right|^{2}}, \\
M_{0}^{(1)}(t, t) & =-\frac{1}{2 \pi}\left|z^{\prime}(t)\right| \\
M_{0}^{(2)}(t, t) & =0
\end{aligned}
$$

ist das lineare Gleichungssystem

$$
f_{j}^{(n)}=\varphi_{j}^{(n)}-\frac{\pi}{n} \sum_{\substack{k=1 \\ k \neq 2 n}}^{4 n-1} \alpha_{k}^{(n)} L_{0}\left(s_{j}^{(n)}, s_{k}^{(n)}\right)\left\{\varphi_{k}^{(n)}-u_{0, k}^{(0)} \varphi_{0}^{(n)}-u_{1, k}^{(0)} \varphi_{2 n}^{(n)}\right\}
$$




$$
\begin{gathered}
+\sum_{l=0}^{1} \varphi_{2 n l}^{(n)}\left\{u_{l, j}^{(0)}-\frac{\pi}{n} \sum_{\substack{k=1 \\
k \neq 2 n}}^{4 n-1} \alpha_{k}^{(n)}\left(M_{0}^{(2)}\left(s_{j}^{(n)}, s_{k}^{(n)}\right)+M_{0}^{(1)}\left(s_{j}^{(n)}, s_{k}^{(n)}\right) \times\right.\right. \\
\left.\left.\times\left[T\left(t_{j}^{(n)}, t_{k}^{(n)}\right)+\frac{n}{\pi} R_{|k-j|}^{(n)}\right]\right) u_{l, k}^{(1)}\right\},
\end{gathered}
$$

$j=0, \ldots, 4 n-1$, zu lösen. Dabei sind $T$ und $R_{k}^{(n)}$ durch (3.18) und (3.21) erklärt.

\subsection{Der hypersinguläre Operator $T_{0}$}

Zur Auswertung der Normalableitung $\left.\frac{\partial u}{\partial \nu}\right|_{\Gamma_{0}}$ wird der Operator $T_{0}$, mit

$$
\left(T_{0} \varphi\right)(x)=2 \frac{\partial}{\partial \nu(x)} \int_{\partial D} \varphi(y) \frac{\partial}{\partial \nu(y)} \Phi_{0}(x, y) d s(y), x \in \partial D \backslash\left\{x^{(0)}, x^{(1)}\right\},
$$

benötigt. Da die Dichte $\varphi$ auf jeder abgeschlossenen Teilmenge von $\Gamma_{0}$ und $\gamma_{0}$ die Regularität $C^{1, \alpha}$ besitzt und sich $\varphi$ bei $x^{(l)}$ wie $\left(x-x^{(l)}\right)^{\delta}, \delta=\frac{\pi}{\pi+\left|\pi-\beta\left(x^{(l)}\right)\right|}$, verhält (siehe Grisvard [15] und [15]), gilt der nachfolgende Satz.

Satz 7.7 Sei z eine Parametrisierung von $\partial D$ wie im Abschnitt 7.1 beschrieben. Sei $\chi \in C(\partial D)$ und gelte $\chi\left(x^{(0)}\right)=\chi\left(x^{(1)}\right)=0$ sowie $\left.\chi \circ z\right|_{[0,2 \pi]},\left.\chi \circ z\right|_{[2 \pi, 4 \pi]} \in S^{2,1+\delta}$ mit $0<\delta<1$. Dann gilt für die Normalableitung des Doppelschichtpotenzials für $x \in \Gamma_{0} \cup \gamma_{0}$ die Darstellung

$$
\frac{\partial}{\partial \nu(x)} \int_{\partial D} \frac{\partial \Phi_{0}(x, y)}{\partial \nu(y)} \chi(y) d s(y)=\int_{\partial D} \frac{\partial \Phi_{0}(x, y)}{\partial t(x)} \frac{\partial \chi}{\partial t}(y) d s(y) .
$$

Dabei ist $t=\left(t_{1}, t_{2}\right)=\left(-\nu_{2}, \nu_{1}\right)$ der Tangentialvektor.

Beweis: Das obige Resultat ist sowohl für geschlossene Ränder $\partial D \in C^{2}$ mit Dichte $\chi \in C^{1, \alpha}(\partial D)$ (siehe Kress [35, Theorem 7.29]), als auch beim offenen Bogen der Klasse $C^{3}$ mit Dichte $\chi \in C^{1, \alpha, *}$ (siehe Mönch [44, Satz 2.8]) bekannt.

Hierbei ist $\chi$ im Funktionenraum $C^{1, \alpha, *}(\Gamma)$ genau dann, wenn die Dichte in den Endpunkten verschwindet, die parametrisierte Dichte $\chi \circ z$ differenzierbar in $\Gamma_{0}$ ist, die Ableitung $(\chi \circ z)^{\prime}$ ein wurzelsinguläres Verhalten an den Endpunkten aufweist und im Inneren hölderstetig ist. 
Mit einer geeigneten Abschneidetechnik wird das Problem auf die bereits bewiesenen Gleichungen reduziert.

Dazu sei $x \in \Gamma_{0}$ fest. Dann existieren Teilbögen $\Gamma_{2}, \Gamma_{1}$ und eine Funktion $\rho \in C^{2}(\Gamma)$ mit den Eigenschaften:

- $\Gamma_{1}$ lässt sich zu einer geschlossenen $C^{2}$-glatten Kurve $\tilde{\Gamma}$ ergänzen.

- $x \in \Gamma_{2} \subset \Gamma_{1} \subset \Gamma_{0}$.

- $\left.\rho\right|_{\Gamma_{2}} \equiv 1,\left.\rho\right|_{\Gamma \backslash \Gamma_{1}} \equiv 0$.

Die Dichte $\chi$ wird aufgespalten in

$$
\chi=\rho \chi+(1-\rho) \chi .
$$

Weil $\rho \chi$ außerhalb von $\Gamma_{1}$ verschwindet, ergibt sich mit dem bereits erwähnten Satz [35., Theorem 7.29], angewendet auf den Rand $\tilde{\Gamma}$,

$$
\frac{\partial}{\partial \nu(x)} \int_{\partial D} \frac{\partial \Phi_{0}(x, y)}{\partial \nu(y)} \rho(y) \chi(y) d s(y)=\int_{\partial D} \frac{\partial \Phi_{0}(x, y)}{\partial t(x)} \frac{\partial(\rho \chi)}{\partial t}(y) d s(y) .
$$

Desgleichen folgt aus [44, Satz 2.8], angewendet auf 3 Bogenstücke ( $\gamma$ sowie den linken und rechten Teil von $\left.\Gamma \backslash \Gamma_{2}\right)$ und die Dichte $(1-\rho) \chi \in C^{1, \alpha, *}$, die Gleichung

$$
\frac{\partial}{\partial \nu(x)} \int_{\partial D} \frac{\partial \Phi_{0}(x, y)}{\partial \nu(y)}(1-\rho(y)) \chi(y) d s(y)=\int_{\partial D} \frac{\partial \Phi_{0}(x, y)}{\partial t(x)} \frac{\partial}{\partial t(y)}((1-\rho) \chi)(y) d s(y) .
$$

Addieren von (7.16) und (7.17) ergibt die Behauptung.

Aus der Darstellung

$$
\begin{aligned}
u(x)= & \int_{\partial D} \frac{\partial \Phi_{0}(x, y)}{\partial \nu(y)} \varphi(y) d s(y) \\
= & \int_{\partial D} \frac{\partial \Phi_{0}(x, y)}{\partial \nu(y)}\left\{\varphi(y)-\sum_{l=0}^{1} \varphi\left(x^{(l)}\right) u_{l}(y)\right\} d s(y) \\
& +\sum_{l=0}^{1} \varphi\left(x^{(l)}\right)\left\{\int_{\partial D} \Phi_{0}(x, y) \frac{\partial u_{l}}{\partial \nu}(y) d s(y)-u_{l}(x)\right\}, x \in D,
\end{aligned}
$$


gewinnt man für die zu ermittelnde Normalableitung

$$
\frac{\partial u}{\partial \nu}(x)=\frac{1}{2}\left(\tilde{T}_{0} \varphi\right)(x)+\sum_{l=0}^{1} \varphi\left(x^{(l)}\right)\left[\frac{1}{2}\left(K_{0}^{*} \frac{\partial u_{l}}{\partial \nu}\right)(x)-\frac{1}{2} \frac{\partial u_{l}}{\partial \nu}(x)\right], x \in \Gamma_{0} .
$$

Hierbei ist $\tilde{T}_{0}$ der Operator, bei dem nicht die Dichte $\varphi$, sondern die in den Ecken verschwindende Dichte $\varphi-\sum_{l=0}^{1} \varphi\left(x^{(l)}\right) u_{l}$ eingetragen wird.

Zur Vereinfachung der Darstellung sei nun $\chi:=\varphi-\sum_{l=0}^{1} \varphi\left(x^{(l)}\right) u_{l}$, also eine Dichte, die nach den o.g. Regularitätsresultaten von Grisvard den Voraussetzungen von Satz 7.7 genügt. Dann gilt für $2 \pi<t<4 \pi$

$$
\left(\tilde{T}_{0} \varphi\right)(z(t))=\frac{1}{\left|z^{\prime}(t)\right|} \frac{1}{2 \pi} \int_{0}^{4 \pi} K_{T_{0}}(t, \tau)(\chi \circ z)^{\prime}(\tau) d \tau,
$$

wobei der auf der Diagonalen $t=\tau$ singuläre Kern $K_{T_{0}}$ gegeben ist durch

$$
K_{T_{0}}(t, \tau)=2 \frac{z^{\prime}(t) \cdot(z(\tau)-z(t))}{|z(t)-z(\tau)|^{2}}, t \neq \tau
$$

Während der Integrand über $[0,2 \pi]$ glatt ist, muss beim zweiten Anteil die Singularität berücksichtigt werden.

Aus diesem Grund wird die Cotangens-Singularität auf nachfolgende Weise abgespalten.

$$
\begin{aligned}
\left(\tilde{T}_{0} \varphi\right)(z(t))= & \frac{1}{\left|z^{\prime}(t)\right|} \frac{1}{2 \pi} \int_{0}^{2 \pi} K_{T_{0}}(t, \tau)(\chi \circ z)^{\prime}(\tau) d \tau \\
& +\frac{1}{\left|z^{\prime}(t)\right|} \frac{1}{2 \pi} \int_{2 \pi}^{4 \pi}\left[K_{T_{0}}(t, \tau)-\cot \frac{\tau-t}{2}\right](\chi \circ z)^{\prime}(\tau) d \tau \\
& +\frac{1}{\left|z^{\prime}(t)\right|} \frac{1}{2 \pi} \int_{2 \pi}^{4 \pi} \cot \frac{\tau-t}{2}(\chi \circ z)^{\prime}(\tau) d \tau .
\end{aligned}
$$

Partielle Integration beim ersten und zweiten Summanden führen für $2 \pi<t<4 \pi$ auf

$$
\begin{aligned}
& \left(\tilde{T}_{0} \varphi\right)(z(t)) \\
& =\frac{1}{\left|z^{\prime}(t)\right|}\left\{\int_{0}^{2 \pi} N_{0}^{(1)}(t, \tau)(\chi \circ z)(\tau) d \tau+\int_{2 \pi}^{4 \pi} N_{0}^{(2)}(t, \tau)(\chi \circ z)(\tau) d \tau\right\} \\
& \quad+\frac{1}{\left|z^{\prime}(t)\right|} \frac{1}{2 \pi} \int_{2 \pi}^{4 \pi} \cot \frac{\tau-t}{2}(\chi \circ z)^{\prime}(\tau) d \tau .
\end{aligned}
$$


Dabei sind die Kerne $N_{0}^{(1)}$ und $N_{0}^{(2)}$ gegeben durch

$$
\begin{aligned}
N_{0}^{(1)}(t, \tau)= & \frac{-1}{2 \pi} \frac{\partial}{\partial \tau} K_{T_{0}}(t, \tau) \\
= & \frac{2}{\pi} \frac{z^{\prime}(t) \cdot(z(t)-z(\tau)) z^{\prime}(\tau) \cdot(z(t)-z(\tau))}{|z(t)-z(\tau)|^{4}}-\frac{1}{\pi} \frac{z^{\prime}(t) \cdot z^{\prime}(\tau)}{|z(t)-z(\tau)|^{2}}, \\
N_{0}^{(2)}(t, \tau)= & \frac{-1}{2 \pi} \frac{\partial}{\partial \tau}\left[K_{T_{0}}(t, \tau)-\cot \frac{\tau-t}{2}\right] \\
= & \frac{2}{\pi} \frac{z^{\prime}(t) \cdot(z(t)-z(\tau)) z^{\prime}(\tau) \cdot(z(t)-z(\tau))}{|z(t)-z(\tau)|^{4}}-\frac{1}{\pi} \frac{z^{\prime}(t) \cdot z^{\prime}(\tau)}{|z(t)-z(\tau)|^{2}} \\
& -\frac{1}{4 \pi} \frac{1}{\sin ^{2} \frac{t-\tau}{2}}, 2 \pi<t<4 \pi, 2 \pi \leq \tau \leq 4 \pi
\end{aligned}
$$

Während die Diagonalterme für $N_{0}^{(1)}$ nicht benötigt werden, ergibt sich für $N_{0}^{(2)}$

$$
N_{0}^{(2)}(t, t)=-\frac{1}{12 \pi}-\frac{\left|z^{\prime}(t) \cdot z^{\prime \prime}(t)\right|^{2}}{2 \pi\left|z^{\prime}(t)\right|^{4}}+\frac{\left|z^{\prime \prime}(t)\right|^{2}}{4 \pi\left|z^{\prime}(t)\right|^{2}}+\frac{z^{\prime}(t) \cdot z^{\prime \prime \prime}(t)}{6 \pi\left|z^{\prime}(t)\right|^{2}} .
$$

Eine Substitution $t=w(s), \tau=w(\sigma)$ in (7.19) und die Vereinbarung

$$
h(s, \sigma):=\left\{\begin{array}{cl}
\frac{\cot \frac{w(\sigma)-w(s)}{2}}{\cot \frac{\sigma-s}{2}} & : 0 \leq s, \sigma \leq 4 \pi,|s-\sigma| \notin\{0,2 \pi, 4 \pi\} \\
\frac{1}{w^{\prime}(\sigma)} & : 2 \pi<s=\sigma<4 \pi
\end{array},\right.
$$

führen für $2 \pi<s<4 \pi \mathrm{zu}$

$$
\begin{aligned}
\left(\tilde{T}_{0} \varphi\right)(z(w(s)))= & \frac{1}{\left|z^{\prime}(w(s))\right|} \int_{0}^{2 \pi} N_{0}^{(1)}(w(s), w(\sigma))(\chi \circ z \circ w)(\sigma) w^{\prime}(\sigma) d \sigma \\
& +\frac{1}{\left|z^{\prime}(w(s))\right|} \int_{2 \pi}^{4 \pi} N_{0}^{(2)}(w(s), w(\sigma))(\chi \circ z \circ w)(\sigma) w^{\prime}(\sigma) d \sigma \\
& +\frac{1}{\left|z^{\prime}(w(s))\right|} \frac{1}{2 \pi} \int_{0}^{2 \pi} \cot \frac{\sigma-s}{2} h(s, \sigma)(\chi \circ z \circ w)^{\prime}(\sigma+2 \pi) d \sigma .
\end{aligned}
$$

Beim letzten Integral wurde von $w(\sigma+2 \pi)=2 \pi+w(\sigma)$ und der Periodizität vom Cotangens Gebrauch gemacht. 
Für den dritten Summanden in (7.24) sind Quadraturformeln

$$
\begin{aligned}
\frac{1}{2 \pi} \int_{0}^{2 \pi} \cot \frac{\sigma-s}{2} \psi^{\prime}(\sigma+2 \pi) d \sigma & \approx \frac{1}{2 \pi} \int_{0}^{2 \pi} \cot \frac{\sigma-s}{2}\left(P_{n} \psi\right)^{\prime}(\sigma) d \sigma \\
\frac{1}{2 \pi} \int_{0}^{2 \pi} \cot \frac{\sigma-s}{2} \psi(\sigma+2 \pi) d \sigma & \approx \frac{1}{2 \pi} \int_{0}^{2 \pi} \cot \frac{\sigma-s}{2}\left(P_{n} \psi\right)(\sigma) d \sigma
\end{aligned}
$$

erforderlich. Dabei ist $P_{n}$ der trigonometrische Interpolationsoperator

$$
P_{n}: C_{2 \pi} \rightarrow T_{n}, P_{n} \psi=\sum_{j=2 n}^{4 n-1} \psi\left(t_{j}^{(n)}\right) l_{j}^{(n)}=\sum_{j=0}^{2 n-1} \psi\left(t_{j+2 n}^{(n)}\right) l_{j}^{(n)}
$$

zu den äquidistanten Knoten $t_{j}^{(n)}=j \pi / n, j=2 n, \ldots, 4 n-1$. Durch exaktes Integrieren von

$$
T_{j}^{(n)}(s)=\frac{1}{2 \pi} \int_{0}^{2 \pi} \cot \frac{\sigma-s}{2} l_{j}^{(n)^{\prime}}(\sigma) d \sigma
$$

und

$$
U_{j}^{(n)}(s)=\frac{1}{2 \pi} \int_{0}^{2 \pi} \cot \frac{\sigma-s}{2} l_{j}^{(n)}(\sigma) d \sigma
$$

ergeben sich die Quadraturformeln (vergleiche Mönch [43, Kapitel 3.1] und Kreß [26])

$$
\begin{aligned}
\frac{1}{2 \pi} \int_{0}^{2 \pi} \cot \frac{\sigma-s}{2} \psi^{\prime}(\sigma+2 \pi) d \sigma & \approx \sum_{j=0}^{2 n-1} \psi\left(t_{j+2 n}^{(n)}\right) T_{j}^{(n)}(s), \\
\frac{1}{2 \pi} \int_{0}^{2 \pi} \cot \frac{\sigma-s}{2} \psi(\sigma+2 \pi) d \sigma & \approx \sum_{j=0}^{2 n-1} \psi\left(t_{j+2 n}^{(n)}\right) U_{j}^{(n)}(s)
\end{aligned}
$$

mit den Quadraturgewichten

$$
\begin{aligned}
T_{j}^{(n)}(s) & =-\frac{1}{n} \sum_{m=1}^{n-1} m \cos m\left(s-t_{j}^{(n)}\right)-\frac{1}{2} \cos n\left(s-t_{j}^{(n)}\right), j=0, \ldots, 2 n-1, \\
U_{j}^{(n)}(s) & =\frac{1}{2 n}\left(1-(-1)^{j} \cos (n s)\right) \cot \frac{t_{j}^{(n)}-s}{2}, j=0, \ldots, 2 n-1 .
\end{aligned}
$$

Mit der abkürzenden Schreibweise

$$
T_{|j-l|}^{(n)}:=T_{j}^{(n)}\left(t_{l}^{(n)}\right), U_{j-l}^{(n)}:=U_{j}^{(n)}\left(t_{l}^{(n)}\right)=-U_{l}^{(n)}\left(t_{j}^{(n)}\right),
$$


ergeben sich die Matrixelemente

$$
\begin{aligned}
& T_{k}^{(n)}=-\frac{1}{n} \sum_{m=1}^{n-1} m \cos \left(\frac{m k \pi}{n}\right)-\frac{(-1)^{k}}{2}, k=0, \ldots, 2 n-1, \\
& U_{k}^{(n)}=\left\{\begin{array}{cl}
0 & : k \text { ist gerade } \\
\frac{1}{n} \cot \frac{k \pi}{2 n} & : \quad k \text { ist ungerade }
\end{array}, k=-2 n+1, \ldots, 2 n-1 .\right.
\end{aligned}
$$

Die Produktregel $h \psi^{\prime}=(h \psi)^{\prime}-h^{\prime} \psi$ motiviert mit den Funktionen $\hat{U}_{j}^{(n)}$,

$$
\hat{U}_{j}^{(n)}(s)=h\left(s, t_{j+2 n}^{(n)}\right) T_{j}^{(n)}(s)-\frac{\partial h}{\partial \sigma}\left(s, t_{j+2 n}^{(n)}\right) U_{j}^{(n)}(s), j=0, \ldots, 2 n-1,
$$

die Approximation

$$
\frac{1}{2 \pi} \int_{0}^{2 \pi} \cot \frac{\sigma-s}{2} h(s, \sigma) \psi^{\prime}(\sigma+2 \pi) d \sigma \approx \sum_{j=0}^{2 n-1} \psi\left(t_{j+2 n}^{(n)}\right) \hat{U}_{j}^{(n)}(s) .
$$

In dieser Arbeit wird stattdessen

$$
\begin{aligned}
\frac{1}{2 \pi} \int_{0}^{2 \pi} \cot \frac{\sigma-s}{2} h(s, \sigma) \psi^{\prime}(\sigma+2 \pi) & \approx \sum_{j=0}^{2 n-1} h\left(s, t_{j+2 n}^{(n)}\right) \psi^{\prime}\left(t_{j+2 n}^{(n)}\right) U_{j}^{(n)}(s) \\
& \approx \sum_{j, k=0}^{2 n-1} h\left(s, t_{j+2 n}^{(n)}\right) \psi\left(t_{k+2 n}^{(n)}\right) l_{k}^{(n)^{\prime}}\left(t_{j+2 n}^{(n)}\right) U_{j}^{(n)}(s)
\end{aligned}
$$

verwendet. Wegen der Singularitäten der Kerne ist nicht zu erwarten, dass obige Quadraturformeln gleichmäßig bezüglich $s \in(2 \pi, 4 \pi)$ konvergieren. Eine gleichmäßige Konvergenz auf kompakten Teilmengen von $(2 \pi, 4 \pi)$ erscheint plausibel.

Insgesamt ergibt sich für den gemäß (7.18) benötigten hypersingulären Anteil die Approximation

$$
\begin{aligned}
\left(\tilde{T}_{0} \varphi\right)(z(w(s))) \approx & \frac{1}{\left|z^{\prime}(w(s))\right|} \frac{\pi}{n} \sum_{j=1}^{2 n-1} \alpha_{j}^{(n)} N_{0}^{(1)}\left(w(s), s_{j}^{(n)}\right) \chi_{j}^{(n)} \\
& +\frac{1}{\left|z^{\prime}(w(s))\right|} \frac{\pi}{n} \sum_{j=2 n+1}^{4 n-1} \alpha_{j}^{(n)} N_{0}^{(2)}\left(w(s), s_{j}^{(n)}\right) \chi_{j}^{(n)} \\
& +\frac{1}{\left|z^{\prime}(w(s))\right|} \sum_{j, k=0}^{2 n-1} h\left(s, t_{j+2 n}^{(n)}\right) l_{k}^{(n)^{\prime}}\left(t_{j+2 n}^{(n)}\right) U_{j}^{(n)}(s) \chi_{k+2 n}^{(n)} .
\end{aligned}
$$




\subsection{Numerische Ergebnisse beim direkten Impedanz- randwertproblem}

In diesem Abschnitt werden die nachfolgenden Parametrisierungen $z_{j}$ als Beispiele für $\gamma$ betrachtet:

$$
\begin{aligned}
& z_{1}(t)=-(\cos (t / 2), \sin (t / 2)), 0 \leq t \leq 2 \pi \\
& z_{2}(t)=\frac{0.5(t-2 \pi)-1}{1+\frac{\pi}{2}}(\cos (t / 2), \sin (t / 2))+\left(\frac{\frac{\pi}{2}}{1+\frac{\pi}{2}}, 0\right), 0 \leq t \leq 2 \pi, \\
& z_{3}(t)=-\frac{1+0.5 t}{1+\frac{\pi}{2}}(\cos (t / 2), \sin (t / 2))-\left(\frac{\frac{\pi}{2}}{1+\frac{\pi}{2}}, 0\right), 0 \leq t \leq 2 \pi, \\
& z_{4}(t)=-\left(1-\frac{1}{3} \sin (t / 2)+\frac{1}{6} \sin (1.5 t)\right)(\cos (t / 2), \sin (t / 2)), 0 \leq t \leq 2 \pi, \\
& z_{5}(t)=-(1+\sin (t / 2))(\cos (t / 2), \sin (t / 2)), 0 \leq t \leq 2 \pi, \\
& z_{6}(t)=\left(\frac{1}{\pi}(t-\pi),-\sin (t / 2)\right), 0 \leq t \leq 2 \pi .
\end{aligned}
$$

Durch Spiegelung an der $x_{1}$-Achse erhält man ebenfalls 6 Parametrisierungen für $\Gamma$, sodass durch Kombination mit denen für $\gamma$ hinreichend viele Gebiete mit Ecken zur Verfügung stehen. In Abbildung 7.1 sind die Kurven, abgesehen von Skalierungsfaktoren in $x_{2^{-}}$

Richtung, dargestellt. Entsprechend der Parametrisierung sind sie mit $\gamma_{j}, \Gamma_{j}, j=1, \ldots, 6$ bezeichnet.

Beispiel 7.8 Der Poisson-Kern

$$
u(x)=\frac{1}{2 \pi} \frac{|y|^{2}-|x|^{2}}{|x-y|^{2}}, x \neq y,
$$

ist harmonisch in $\mathbb{R}^{2} \backslash\{y\}$. Für $\gamma$ wird der Halbkreis (7.31) verwendet. Für $\Gamma_{6}$ wähle $y=0.5 \sqrt{2}(1,1)^{t}$ und in den anderen Fällen setze $y=(0,1)^{t}$, wobei für $\Gamma_{1}, \Gamma_{2}, \Gamma_{3}$ und $\Gamma_{5}$ noch ein geeigneter Skalierungsfaktor (hier $1 / 3$ ) für die $x_{2}$-Richtung zu wählen ist, damit die Singularität y außerhalb des Gebietes D liegt. In allen Fällen hat u auf $\gamma$ homogene Dirichletwerte. Es gilt

$$
\operatorname{grad} u(x)=\frac{-1}{\pi}\left[\frac{\left(|y|^{2}-|x|^{2}\right)(x-y)}{|x-y|^{4}}+\frac{x}{|x-y|^{2}}\right], x \neq y .
$$


Abbildung 7.1: Darstellungen von $\gamma_{j}$ und $\Gamma_{j}$.
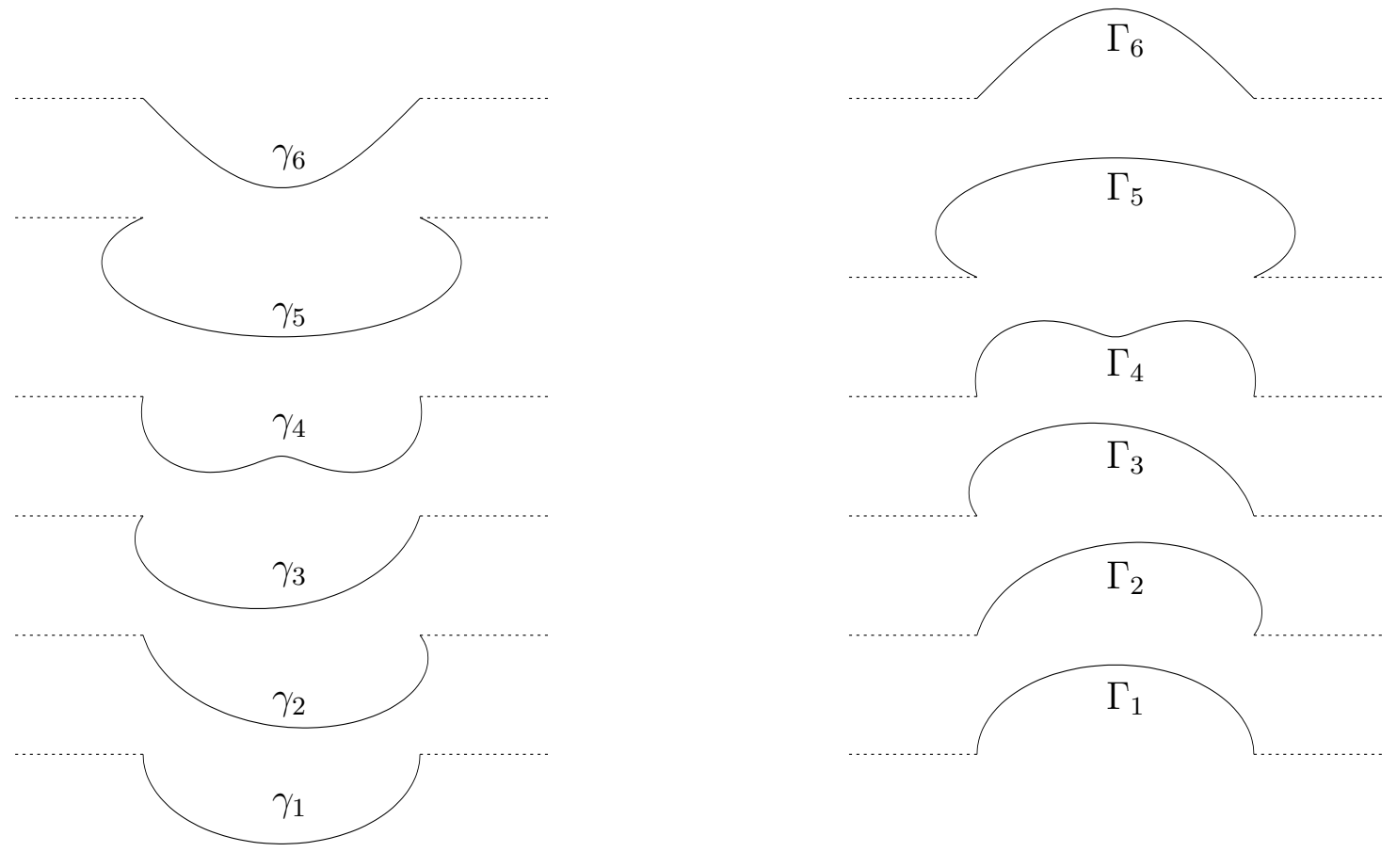

In Tabelle 7.1 ist für verschiedene Punkte $x \in \Gamma$ die Differenz zwischen exakter Lösung $\frac{\partial u}{\partial \nu}(x)$ und dem numerisch ermittelten Wert dargestellt. Die erwartete schnelle Konvergenz ist ersichtlich, obwohl die Lösung eine Singularität in der Nähe des Randes besitzt.

Beispiel 7.9 Die in Polarkoordinaten $(r, \vartheta) \in \mathbb{R}^{+} \times[-\pi, \pi)$ dargestellte Funktion

$$
u(r, \vartheta)=r^{\alpha} \sin (\alpha \vartheta), \alpha>0,
$$

ist harmonisch in $\mathbb{R}^{2} \backslash\left\{y \in \mathbb{R}^{2} \mid y_{1} \leq 0, y_{2}=0\right\}$. Durch Transformation des Koordinatenursprungs in die Ecke $x^{(0)}$ ist die Lösung mit den durch (7.37) gegebenen Randwerten in der Regel $(\alpha<1)$ in $x^{(0)}$ nicht mehr differenzierbar. Es gilt

$$
\operatorname{grad} u(x)=\frac{\partial u}{\partial r}(\cos \vartheta, \sin \vartheta)^{t}+\frac{1}{r} \frac{\partial u}{\partial \vartheta}(-\sin \vartheta, \cos \vartheta)^{t}, x \neq(0,0)^{t} .
$$

In Tabelle 7.9 ist für Punkte $x \in \Gamma$ (nicht zu nahe bei $\left.x^{(0)}\right)$ der Fehler zwischen exakter Lösung und Näherungslösung dargestellt. Die Ergebnisse sind mit dem unteren Rand 
(7.34) und $\alpha=1.5$ erzeugt worden. Die Konvergenz ist in allen Beispielen ersichtlich. Aus weiteren hier nicht angeführten numerischen Ergebnissen kann der Einfluss von $\alpha$ auf die Konvergenzgeschwindigkeit abgelesen werden.

Beispiel 7.10 Die durch

$$
u(y)=y_{1}^{2}-y_{2}^{2}, y \in \mathbb{R}^{2},
$$

gegebene Funktion ist harmonisch mit dem Gradienten

$$
\operatorname{grad} u(y)=\left(2 y_{1},-2 y_{2}\right)^{t} \text {. }
$$

Die numerischen Ergebnisse zu diesem Beispiel sind der Tabelle 7.3 zu entnehmen, wobei als unterer Rand die Parametrisierung (7.35) benutzt wurde. 
Tabelle 7.1: Ergebnisse zu Beispiel 7.8.

Substitution $w$ mit kubischem $v$ von Kreß

\begin{tabular}{|c|c|c|c|c|c|}
\hline$p$ & $n$ & $\begin{array}{c}\Gamma_{1} \\
x=z_{1}(3 \pi)\end{array}$ & $\begin{array}{c}\Gamma_{2} \\
x=z_{2}(3 \pi)\end{array}$ & $\begin{array}{c}\Gamma_{4} \\
x=z_{4}(3.5 \pi)\end{array}$ & $\begin{array}{c}\Gamma_{5} \\
x=z_{5}(2.5 \pi)\end{array}$ \\
\hline 3 & $\begin{array}{r}8 \\
16 \\
32 \\
64\end{array}$ & $\begin{array}{l}0.04088360 \\
0.00074931 \\
0.00000031 \\
0.00000002\end{array}$ & $\begin{array}{l}0.05049941 \\
0.00100419 \\
0.00000783 \\
0.00000009\end{array}$ & $\begin{array}{l}0.17503877 \\
0.01842683 \\
0.00004801 \\
0.00000001\end{array}$ & $\begin{array}{l}0.96828029 \\
0.53299242 \\
0.08639986 \\
0.00110266\end{array}$ \\
\hline 4 & $\begin{array}{r}8 \\
16 \\
32 \\
64\end{array}$ & $\begin{array}{l}0.04133535 \\
0.00071517 \\
0.00000013 \\
0.00000000\end{array}$ & $\begin{array}{l}0.05309112 \\
0.00097180 \\
0.00000725 \\
0.00000007\end{array}$ & $\begin{array}{l}0.19044679 \\
0.02108091 \\
0.00005583 \\
0.00000000\end{array}$ & $\begin{array}{l}0.92087003 \\
0.50384811 \\
0.08132141 \\
0.00103137\end{array}$ \\
\hline 5 & $\begin{array}{r}8 \\
16 \\
32 \\
64\end{array}$ & $\begin{array}{l}0.04158528 \\
0.00067048 \\
0.00000011 \\
0.00000000\end{array}$ & $\begin{array}{l}0.05423901 \\
0.00096883 \\
0.00000685 \\
0.00000002\end{array}$ & $\begin{array}{l}0.21091141 \\
0.02464884 \\
0.00006677 \\
0.00000000\end{array}$ & $\begin{array}{l}0.90181860 \\
0.49011772 \\
0.07873004 \\
0.00099171\end{array}$ \\
\hline
\end{tabular}


Tabelle 7.2: Ergebnisse zu Beispiel 7.9.

Substitution $w$ mit kubischem $v$ von Kreß

\begin{tabular}{|c|c|c|c|c|c|}
\hline$p$ & $n$ & $\begin{array}{c}\Gamma_{2} \\
x=z_{2}(3 \pi)\end{array}$ & $\begin{array}{c}\Gamma_{4} \\
x=z_{4}(3 \pi)\end{array}$ & $\begin{array}{c}\Gamma_{5} \\
x=z_{5}(3.5 \pi)\end{array}$ & $\begin{array}{c}\Gamma_{6} \\
x=z_{6}(2.5 \pi)\end{array}$ \\
\hline 3 & $\begin{array}{r}8 \\
16 \\
32 \\
64\end{array}$ & $\begin{array}{l}0.22945000 \\
0.08930281 \\
0.01503920 \\
0.00411923\end{array}$ & $\begin{array}{l}0.07289228 \\
0.00010944 \\
0.00000242 \\
0.00000024\end{array}$ & $\begin{array}{l}0.02033373 \\
0.01237641 \\
0.00693285 \\
0.00361821\end{array}$ & $\begin{array}{l}0.02378610 \\
0.01045901 \\
0.00490836 \\
0.00018632\end{array}$ \\
\hline 4 & $\begin{array}{r}8 \\
16 \\
32 \\
64\end{array}$ & $\begin{array}{l}0.23192281 \\
0.08891620 \\
0.00942235 \\
0.00030716\end{array}$ & $\begin{array}{l}0.08646251 \\
0.00006236 \\
0.00000011 \\
0.00000000\end{array}$ & $\begin{array}{l}0.01853098 \\
0.01033554 \\
0.00534853 \\
0.00229175\end{array}$ & $\begin{array}{l}0.02380779 \\
0.00971269 \\
0.00048331 \\
0.00000952\end{array}$ \\
\hline 5 & $\begin{array}{r}8 \\
16 \\
32 \\
64\end{array}$ & $\begin{array}{l}0.24106712 \\
0.08734712 \\
0.00536076 \\
0.00021094\end{array}$ & $\begin{array}{l}0.10780456 \\
0.00005093 \\
0.00000000 \\
0.00000000\end{array}$ & $\begin{array}{l}0.01159671 \\
0.00613904 \\
0.00215999 \\
0.00029054\end{array}$ & $\begin{array}{l}0.02414860 \\
0.00915583 \\
0.00033804 \\
0.00000053\end{array}$ \\
\hline
\end{tabular}


Tabelle 7.3: Ergebnisse zu Beispiel 7.10.

Substitution $w$ mit kubischem $v$ von Kreß

\begin{tabular}{|c|c|c|c|c|c|}
\hline$p$ & $n$ & $\begin{array}{c}\Gamma_{2} \\
x=z_{2}(3 \pi)\end{array}$ & $\begin{array}{c}\Gamma_{4} \\
x=z_{4}(3 \pi)\end{array}$ & $\begin{array}{c}\Gamma_{5} \\
x=z_{5}(3.5 \pi)\end{array}$ & $\begin{array}{c}\Gamma_{6} \\
x=z_{6}(2.5 \pi)\end{array}$ \\
\hline 3 & $\begin{array}{r}8 \\
16 \\
32 \\
64\end{array}$ & $\begin{array}{l}0.51023910 \\
0.00360023 \\
0.00005687 \\
0.00000105\end{array}$ & $\begin{array}{l}0.13156863 \\
0.00007646 \\
0.00000010 \\
0.00000000\end{array}$ & $\begin{array}{l}0.00129772 \\
0.00000002 \\
0.00000000 \\
0.00000000\end{array}$ & $\begin{array}{l}0.00009755 \\
0.00002093 \\
0.00000260 \\
0.00000029\end{array}$ \\
\hline 4 & $\begin{array}{r}8 \\
16 \\
32 \\
64\end{array}$ & $\begin{array}{l}0.52840933 \\
0.00370941 \\
0.00005327 \\
0.00000087\end{array}$ & $\begin{array}{l}0.16794200 \\
0.00006325 \\
0.00000000 \\
0.00000000\end{array}$ & $\begin{array}{l}0.00861640 \\
0.00000084 \\
0.00000000 \\
0.00000000\end{array}$ & $\begin{array}{l}0.00094984 \\
0.00000114 \\
0.00000005 \\
0.00000000\end{array}$ \\
\hline 5 & $\begin{array}{r}8 \\
16 \\
32 \\
64\end{array}$ & $\begin{array}{l}0.53700627 \\
0.00371588 \\
0.00005004 \\
0.00000067\end{array}$ & $\begin{array}{l}0.21474953 \\
0.00012555 \\
0.00000000 \\
0.00000000\end{array}$ & $\begin{array}{l}0.02691411 \\
0.00000460 \\
0.00000000 \\
0.00000000\end{array}$ & $\begin{array}{l}0.00128323 \\
0.00000075 \\
0.00000000 \\
0.00000000\end{array}$ \\
\hline
\end{tabular}




\section{Kapitel 8}

\section{Das inverse Impedanzrandwertproblem}

In diesem Kapitel wird das inverse Impedanzrandwertproblem behandelt. Es ist die Gestalt des Körpers $D$ unter Kenntnis des oberen Randes $\Gamma$ zu ermitteln. Als Daten stehen Paare $\left(\left.u\right|_{\Gamma},\left.\frac{\partial u}{\partial \nu}\right|_{\Gamma_{0}}\right)$ zur Verfügung. Im gesamten Kapitel 8 werden nur zulässige Gebiete $D \subset \mathbb{R}^{2}$, wie zu Beginn von Kapitel 7 erläutert, betrachtet.

Im Abschnitt 8.1 wird das Problem der Eindeutigkeit untersucht, im Abschnitt 8.2 die Fréchet-Differenzierbarkeit des Randwerteoperators gezeigt und die Ableitung durch ein Randwertproblem charakterisiert. Im letzten Teil des Kapitels werden numerische Beispiele vorgestellt.

\subsection{Eindeutigkeit beim inversen Impedanzrandwert- problem}

Satz 8.1 Es seien $D_{1}$ und $D_{2}$ zwei Gebiete mit gemeinsamen oberen Rand $\Gamma$ und jeweiligem unteren Rand $\gamma_{j}$. Weiterhin seien $\tilde{\Gamma}$ ein nichtleeres zusammenhängendes Teilstück von $\Gamma_{0}$ und

$$
u_{j} \in C^{2}\left(D_{j}\right) \cap C\left(\overline{D_{j}}\right) \cap C^{1}\left(\overline{D_{j}} \backslash\left\{x^{(0)}, x^{(1)}\right\}\right), j=1,2,
$$

Lösungen von

$$
\triangle u_{j}=0 \quad \text { in } D_{j}
$$




$$
\begin{aligned}
u_{1} & =u_{2} \not \equiv 0 \quad \text { auf } \Gamma, \\
\frac{\partial u_{1}}{\partial \nu} & =\frac{\partial u_{2}}{\partial \nu} \quad \text { auf } \tilde{\Gamma} \subset \Gamma_{0}, \\
u_{j} & =0 \quad \text { auf } \gamma_{j} .
\end{aligned}
$$

Dann stimmen die Gebiete $D_{1}$ und $D_{2}$ überein.

Beweis: 1. Für die Parametrisierungen $z^{\left(\gamma_{j}\right)}$ und $z^{(\Gamma)}$ gilt nach Voraussetzung

$$
z_{2}^{\left(\gamma_{j}\right)}(t)<0<z_{2}^{(\Gamma)}(t+2 \pi), 0<t<2 \pi .
$$

Demnach ist $D:=D_{1} \cap D_{2}$ ein nichtleeres Gebiet. Die auf $D$ erklärte Funktion $u:=u_{1}-u_{2}$ ist harmonisch und hat auf $\tilde{\Gamma}$ Cauchy-Randwerte Null. Nach dem Satz von Holmgren (siehe Rauch [4.9, Section 1.7]) verschwindet $u$ in $D$.

2. Angenommen es gilt $\gamma_{1} \neq \gamma_{2}$.

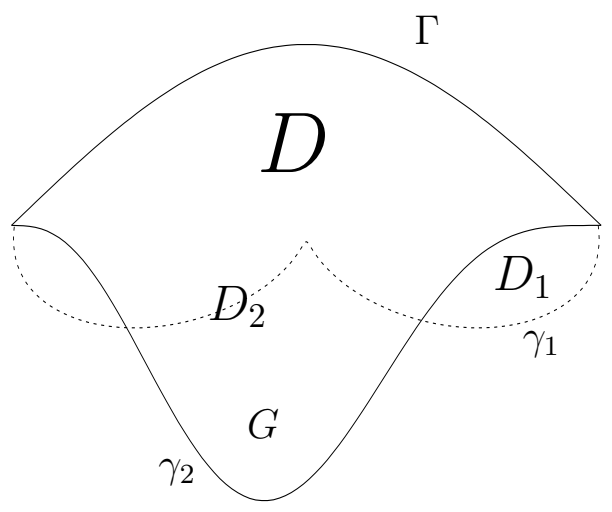

Dann existiert ein $t_{0} \in \mathbb{R}$ so, dass die Gerade durch $\left(t_{0}, 0\right)$ mit Richtungsvektor $(0,1)$ die Kurven $\gamma_{j}$ in Punkten $P_{j}, j=1,2$, schneidet, wobei $P_{1} \neq P_{2}$ gilt. Demnach existiert ein Gebiet $G$ so, dass ohne Beeinträchtigung der Allgemeinheit gilt

$$
G \subset D_{2} \backslash D_{1} \text { und } \partial G \subset \gamma_{1} \cup \gamma_{2} \text {. }
$$

(Anmerkung: Falls sich $\gamma_{j}$ durch $\left(t, z_{2}^{\left(\gamma_{j}\right)}(t)\right), 0 \leq t \leq 2 \pi, j=1,2$, parametrisieren lässt, so seien $0 \leq t_{A}<t_{E} \leq 2 \pi$ mit

$$
\begin{gathered}
\left|z_{2}^{\left(\gamma_{1}\right)}(t)-z_{2}^{\left(\gamma_{2}\right)}(t)\right|>0, \quad t_{A}<t<t_{B}, \\
z_{2}^{\left(\gamma_{1}\right)}\left(t_{A}\right)=z_{2}^{\left(\gamma_{2}\right)}\left(t_{A}\right) \\
z_{2}^{\left(\gamma_{1}\right)}\left(t_{E}\right)=z_{2}^{\left(\gamma_{2}\right)}\left(t_{E}\right) .
\end{gathered}
$$

Dann erfüllt $G$ mit Rand

$$
\partial G=\left\{\max _{j=1,2} z_{2}^{\left(\gamma_{j}\right)}(t) \mid t_{A} \leq t \leq t_{E}\right\} \cup\left\{\min _{j=1,2} z_{2}^{\left(\gamma_{j}\right)}(t) \mid t_{A} \leq t \leq t_{E}\right\} .
$$


obige Forderung.)

Die Funktion $u_{2}$ ist auf $G$ definiert und harmonisch. Auf $\partial G \cap \gamma_{2}$ verschwindet $u_{2}$ nach Voraussetzung. Auf $\partial G \cap \gamma_{1}$ gilt nach Teil 1

$$
\left.u_{2}\right|_{\partial G \cap \gamma_{1}}=\left.u_{1}\right|_{\partial G \cap \gamma_{1}}=0 .
$$

Das Maximum-Minimum Prinzip in $G$ und die Analytizität in $D_{2}$ liefern $u_{2}=0$ in $\overline{D_{2}}$ und daher einen Widerspruch zur Voraussetzung, nach der $u_{2}$ auf $\Gamma$ nicht identisch verschwindet.

\subsection{Die Fréchet - Ableitung des Randwerteoperators}

In diesem Abschnitt soll bei festem $f \in C_{0}^{2, \alpha}(\Gamma):=\left\{g \in C^{2, \alpha}(\Gamma) \mid g\left(x^{(0)}\right)=g\left(x^{(1)}\right)=0\right\}$ die Fréchet-Differenzierbarkeit der Abbildung

$$
z \longmapsto \frac{\partial u}{\partial \nu}
$$

gezeigt werden, wobei die zugehörigen normierten Räume noch zu konkretisieren sind. (Die höhere Regularität von $f$, nämlich $C^{2, \alpha}$ statt $C^{1, \alpha}$ wird benötigt, damit eine Beziehung zwischen $\mathcal{T}_{0}^{\prime}(z, h)$ und der Normalableitung von der Fréchet-Ableitung des Doppelschichtpotenzials genutzt werden kann.)

Dazu sei $\tilde{\Gamma} \subset \Gamma_{0}$ ein zusammenhängendes abgeschlossenes Kurvenstück von $\Gamma_{0}$ mit der Eigenschaft

$$
\operatorname{dist}\left(\tilde{\Gamma}, x^{(j)}\right) \geq C>0, j=0,1
$$

mit einer Konstanten $C$. Weiterhin sei $\hat{z}$ eine Funktion mit den Eigenschaften:

- $\hat{z} \in C_{4 \pi}\left(\mathbb{R}, \mathbb{R}^{2}\right), \hat{z}$ ist injektiv auf $[0,4 \pi)$ und gegen den Uhrzeigersinn orientiert,

- $\hat{z}(0)=x^{(0)}, \hat{z}(2 \pi)=x^{(1)},\left.\hat{z}\right|_{[0,2 \pi]} \in C^{3, \alpha}[0,2 \pi], \hat{z}([2 \pi, 4 \pi])=\Gamma$,

- $\left|\hat{z}^{\prime}(t)\right|>0$ auf $[0,4 \pi]$ und $\hat{z}_{2}(t)<0,0<t<2 \pi$.

Desweiteren werden die Räume $X$ und $U$ erklärt durch

$$
\begin{gathered}
X=\left\{z \in C_{4 \pi}\left(\mathbb{R}, \mathbb{R}^{2}\right)\left|z(t)=(0,0) \in \mathbb{R}^{2} \forall 2 \pi \leq t \leq 4 \pi, z\right|_{[0,2 \pi]} \in C^{3, \alpha}\right\}, \\
U=\left\{z \in X \mid z \text { ist injektiv auf }[0,2 \pi], z_{2}(t)<0 \text { für alle } 0<t<2 \pi\right\} \\
\left.\left|z^{\prime}(t)\right|>0,0 \leq t \leq 2 \pi .\right\}
\end{gathered}
$$


und mit der Norm

$$
\|z\|_{X}=\max \left\{\|z\|_{\infty},\left\|z^{\prime}\right\|_{\infty},\left\|z^{\prime \prime}\right\|_{\infty},\left\|z^{\prime \prime \prime}\right\|_{\infty}\right\}
$$

versehen. $U$ ist offen in $X$.

Für eine zulässige Parametrisierung $z \in U$ werden nun, um (einheitlich) Integrale über geschlossenen Rändern zu erhalten, die auftretenden Operatoren zum Rand $z+\hat{z}$ betrachtet. Unter dem Symbol $\partial D_{z+\hat{z}}$ wird demzufolge

$$
\partial D_{z+\hat{z}}=\{z(t)+\hat{z}(t) \mid 0 \leq t \leq 4 \pi\}
$$

verstanden. Bei der Notation der Kerne $L_{0}, M_{0}, M_{0}^{(1)}$ und $M_{0}^{(2)}$ gemäß (7.7) - (7.10) wird die Parametrisierung mit aufgenommen, das heißt

$$
\begin{aligned}
L_{0}(t, \tau, z+\hat{z}) & =\frac{1}{\pi} \frac{(z+\hat{z})^{\prime}(\tau)^{\perp} \cdot((z+\hat{z})(t)-(z+\hat{z})(\tau))}{|(z+\hat{z})(t)-(z+\hat{z})(\tau)|^{2}}, t \neq \tau, \\
L_{0}(t, t, z+\hat{z}) & =\frac{1}{2 \pi} \frac{(z+\hat{z})^{\prime}(t)^{\perp} \cdot(z+\hat{z})^{\prime \prime}(t)}{\left|(z+\hat{z})^{\prime}(t)\right|^{2}}, \\
M_{0}(t, \tau, z+\hat{z}) & =\frac{1}{\pi} \ln \frac{1}{|(z+\hat{z})(t)-(z+\hat{z})(\tau)|}\left|(z+\hat{z})^{\prime}(\tau)\right| \\
& =M_{0}^{(1)}(t, \tau, z+\hat{z}) \ln \left(4 \sin ^{2} \frac{t-\tau}{2}\right)+M_{0}^{(2)}(t, \tau, z+\hat{z}) .
\end{aligned}
$$

Wie im Kapitel 5 wird zunächst der zur Gleichung (7.4) gehörende Randintegraloperator in parametrisierter Form diskutiert.

Dazu sei $A_{0}(z) \in \mathcal{L}\left(C_{4 \pi}, C_{4 \pi}\right)$ gegeben durch

$$
\left(A_{0}(z) \varphi\right)(t):=-\left(1-\frac{\beta(z(t)+\hat{z}(t))}{\pi}\right) \varphi(t)+\int_{0}^{4 \pi} L_{0}(t, \tau, z+\hat{z}) \varphi(\tau) d \tau, 0 \leq t \leq 4 \pi .
$$

Für den Nachweis der Existenz von $A_{0}^{\prime}(z, h)$ und eine geeignete Darstellung dieser Ableitung werden weitere Operatoren eingeführt. Analog zum Einfachschichtpotenzial $S_{k}(z)$ zur Helmholtzgleichung gemäß (5.6) wird in diesem Abschnitt der Operator $S_{0}(z) \in$ $\mathcal{L}\left(C_{4 \pi}, C_{4 \pi}\right)$ benötigt, wobei gilt

$$
\left(S_{0}(z) \varphi\right)(t):=\int_{0}^{4 \pi} M_{0}(t, \tau, z+\hat{z}) \varphi(\tau) d \tau, 0 \leq t \leq 4 \pi, \varphi \in C_{4 \pi} .
$$


Es wird angemerkt, dass $S_{0}(z)$ der Einfachschichtpotenzialoperator ist, bei dem (abweichend zum Kapitel 5) nicht eine konstante Dichte subtrahiert wird. Neben dem bereits bekannten Raum $C_{4 \pi}$ wird unter $C_{4 \pi}$ null der Teilraum verstanden, bei denen die Funktionen in 0 und $2 \pi$ verschwinden.

Weiterhin wird unter $z \mapsto \frac{\partial u_{l}}{\partial \nu}(z)$ ebenfalls ein Operator verstanden, das heißt

$$
\left(\frac{\partial u_{l}}{\partial \nu}(z)\right)(\tau)=\nu((z+\hat{z})(\tau)) \cdot \operatorname{grad} u_{l}((z+\hat{z})(\tau)), \tau \notin 2 \pi \mathbb{Z} .
$$

Es gelte

$$
\begin{aligned}
\left(B_{0}(z) \varphi\right)(t) & :=\int_{0}^{4 \pi} L_{0}(t, \tau, z+\hat{z}) \varphi(\tau) d \tau, 0 \leq t \leq 4 \pi, \varphi \in C_{4 \pi \text { null }} \\
\left(B_{1}(z) \varphi\right)(t) & :=\varphi(t)-\sum_{l=0}^{1} \varphi(2 \pi l) u_{l}((z+\hat{z})(t)), 0 \leq t \leq 4 \pi, \varphi \in C_{4 \pi} \\
(C(z) \varphi)(t) & :=\sum_{l=0}^{1} \varphi(2 \pi l)\left[\left(S_{0}(z) \frac{\partial u_{l}}{\partial \nu}(z)\right)(t)-u_{l}((z+\hat{z})(t))\right], 0 \leq t \leq 4 \pi, \varphi \in C_{4 \pi}
\end{aligned}
$$

Die Operatoren wurden gerade so gewählt, dass einerseits

$$
A_{0}(z)=B_{0}(z) \circ B_{1}(z)+C(z)
$$

gilt und andererseits die Fréchet-Differenzierbarkeit leicht abzulesen ist.

Satz 8.2 Die Abbildungen $U \rightarrow \mathcal{L}\left(C_{4 \pi \text { null }}, C_{4 \pi}\right), z \mapsto B_{0}(z), U \rightarrow \mathcal{L}\left(C_{4 \pi}, C_{4 \pi \text { null }}\right), z \mapsto$ $B_{1}(z)$ und $U \rightarrow \mathcal{L}\left(C_{4 \pi}, C_{4 \pi}\right), z \mapsto C(z)$ bzw. $z \mapsto A_{0}(z)$ sind Fréchet-differenzierbar. Die Ableitungen sind gegeben durch

$$
\begin{aligned}
\left(B_{0}^{\prime}(z, h) \varphi\right)(t) & =\int_{0}^{4 \pi} \frac{\partial L_{0}}{\partial z}(t, \tau, z+\hat{z}, h) \varphi(\tau) d \tau \\
\left(B_{1}^{\prime}(z, h) \varphi\right)(t) & =-\sum_{l=0}^{1} \varphi(2 \pi l) \operatorname{grad} u_{l}((z+\hat{z})(t)) \cdot h(t), \\
\left(C^{\prime}(z, h) \varphi\right)(t) & =\sum_{l=0}^{1} \varphi(2 \pi l)\left[\left(S_{0}^{\prime}(z, h) \frac{\partial u_{l}}{\partial \nu}(z)\right)(t)+\left(S_{0}(z) \frac{\partial}{\partial z} \frac{\partial u_{l}}{\partial \nu}(z, h)\right)(t)\right. \\
A_{0}^{\prime}(z, h) & =B_{0}^{\prime}(z, h) \circ B_{1}(z)+B_{0}(z) \circ B_{1}^{\prime}(z, h)+C^{\prime}(z, h) .
\end{aligned}
$$


Beweis: Für $B_{1}(z)$ ist die Aussage offensichtlich. Bei den Operatoren $B_{0}(z)$ und $C(z)$ wird auf den Differenziationssatz 5.2 , Lemma 5.8 sowie die Sätze 5.9 und 5.10 verwiesen. (Für $B_{0}(z)$ ist der Definitionsbereich $C_{4 \pi}$ null erforderlich.)

Die Aussage für $A_{0}(z)$ folgt aus der Produktregel Satz 2.3.

Der nachfolgende Satz kann als Analogon zu Satz 5.11 angesehen werden.

Satz 8.3 Seien $z_{0} \in U$ und $G \subset \mathbb{R}^{2}$ ein Gebiet mit $\bar{G} \subset \mathbb{R}^{2} \backslash \partial D_{z_{0}+\hat{z}}$. Für hinreichend kleines $R>0$ sei z aus $B\left(z_{0}, R\right) \subset U . P(z)$ bezeichne den Potenzialoperator

$$
P(z): C_{4 \pi}(\mathbb{R}, \mathbb{R}) \longrightarrow C_{b}(G)
$$

in parametrisierter Form

$$
(P(z) \psi)(x)=\int_{0}^{4 \pi} f_{K_{0}}(x, \tau, z+\hat{z}) \psi(\tau) d \tau, x \in G,
$$

mit dem auf $G \times[0,4 \pi] \times\left\{z \mid z-\hat{z} \in B\left(z_{0}, R\right) \subset U\right\}$ erklärten Kern

$$
f_{K_{0}}(x, \tau, z+\hat{z})=\frac{1}{2 \pi} \frac{(z+\hat{z})^{\prime}(\tau)^{\perp} \cdot(x-z(\tau)-\hat{z}(\tau))}{|x-(z+\hat{z})(\tau)|^{2}} .
$$

Die Abbildung $z \mapsto P(z)$ ist Fréchet-differenzierbar von $B\left(z_{0}, R\right) \subset U$ nach $\mathcal{L}\left(C_{4 \pi}(\mathbb{R}, \mathbb{R}), C_{b}(G)\right)$ mit Ableitung

$$
\begin{aligned}
& X \longrightarrow \mathcal{L}\left(C_{4 \pi}(\mathbb{R}, \mathbb{R}), C_{b}(G)\right), h \mapsto P^{\prime}(z, h), \\
& \left(P^{\prime}(z, h) \psi\right)(x)=\int_{0}^{4 \pi} \frac{\partial}{\partial z} f_{K_{0}}(x, \tau, z+\hat{z}, h) \psi(\tau) d \tau, x \in G .
\end{aligned}
$$

Beweis: Siehe Bemerkung 5.5 zum Satz 5.2 .

Desweiteren wird ein Operator benötigt, der ein gegebenes $f \in C_{0}^{2, \alpha}(\Gamma)$ zu einer auf einer geschlossenen Kurve stetigen Funktion fortsetzt.

Lemma 8.4 Für $z \in U$ wird $R(z): C_{0}^{2, \alpha}(\Gamma) \rightarrow C_{4 \pi}(\mathbb{R}, \mathbb{R})$ erklärt durch

$$
(R(z) f)(t):=\left\{\begin{array}{cl}
0 & : 0 \leq t \leq 2 \pi, \\
-2 f(z(t)+\hat{z}(t)) & : \quad 2 \pi \leq t \leq 4 \pi .
\end{array}\right.
$$

Dann ist $z \mapsto R(z)$ Fréchet-differenzierbar von $U$ nach $\mathcal{L}\left(C_{0}^{2, \alpha}(\Gamma), C_{4 \pi}(\mathbb{R}, \mathbb{R})\right)$ mit Ableitung $R^{\prime}(z, h)=0 \in C_{4 \pi}(\mathbb{R}, \mathbb{R})$. 
Beweis: Für $z \in U$ und $h \in X$ gilt $z(t)=h(t)=0,2 \pi \leq t \leq 4 \pi$, also $R(z+h)=R(z)$.

Als nächstes soll der Operator $T_{0}$ in parametrisierter Form betrachtet werden. Dazu wird an die Darstellung (7.19) mit den Kernen $N_{0}^{(1)}, N_{0}^{(2)}$ gemäß (7.20) - (7.22) erinnert.

Wegen der Identität (siehe (7.18))

$$
T_{0} \varphi=\tilde{T}_{0} \varphi+\sum_{l=0}^{1} \varphi\left(x^{(l)}\right)\left[K_{0}^{*} \frac{\partial u_{l}}{\partial \nu}-\frac{\partial u_{l}}{\partial \nu}\right] \text { auf } \Gamma_{0}
$$

ergibt sich für die parametrisierte Form

$$
\mathcal{T}_{0}(z)=\mathcal{T}_{0,1}(z) \circ B_{2}(z)+\mathcal{T}_{0,2}(z)
$$

mit den Operatoren

$$
\begin{aligned}
\left(B_{2}(z) \varphi\right)(\tau)= & \frac{\partial}{\partial \tau}\left(B_{1}(z) \varphi\right)(\tau) \\
= & \varphi^{\prime}(\tau)-\sum_{l=0}^{1} \varphi(2 \pi l) \operatorname{grad} u_{l}((z+\hat{z})(\tau)) \cdot(z+\hat{z})^{\prime}(\tau), \\
& 0 \leq \tau \leq 4 \pi, \varphi \in C_{4 \pi}^{1, \alpha} \\
\left(\mathcal{T}_{0,1} \varphi\right)(t)= & \frac{1}{\left|(z+\hat{z})^{\prime}(t)\right|} \frac{1}{2 \pi} \int_{2 \pi}^{4 \pi} \cot \frac{\tau-t}{2} \varphi(\tau) d \tau, t_{a} \leq t \leq t_{e}, \varphi \in C_{4 \pi}^{0, \alpha}, \\
\left(\mathcal{T}_{0,2} \varphi\right)(t)= & \frac{1}{\left|(z+\hat{z})^{\prime}(t)\right|}\left[\int_{0}^{2 \pi} N_{0}^{(1)}(t, \tau, z+\hat{z})\left(B_{1}(z) \varphi\right)(\tau) d \tau+\right. \\
& +\sum_{l=0}^{1} \varphi(2 \pi l)\left[\left(A_{0}^{*}(z) \frac{\partial u_{l}}{\partial \nu}(z)\right)(t)-\left(\frac{\partial u_{l}}{\partial \nu}(z)\right)(t)\right], \\
& t_{a} \leq t \leq t_{e}, \varphi \in C_{4 \pi}^{1, \alpha} .
\end{aligned}
$$

Hierbei sind $2 \pi<t_{a}<t_{e}<4 \pi$ die eindeutig bestimmten Zahlen mit $\hat{z}\left(\left[t_{a}, t_{e}\right]\right)=\tilde{\Gamma}$ und $A_{0}^{*}(z)$ im Wesentlichen der durch Vertauschen der Kernvariablen bei $L_{0}$ aus $A_{0}(z)$ entstehende Operator. Es sind aber die Definitions- und Wertebereiche von $A_{0}(z)$ und $A_{0}^{*}(z)$ verschieden. Insbesondere gilt für $t_{a} \leq t \leq t_{e}$

$\left(A_{0}^{*}(z) \frac{\partial u_{l}}{\partial \nu}(z)\right)(t)=\int_{0}^{4 \pi} L_{0}(\tau, t, z+\hat{z}) \frac{\left|(z+\hat{z})^{\prime}(\tau)\right|}{\left|(z+\hat{z})^{\prime}(t)\right|} \frac{(z+\hat{z})^{\prime}(\tau)^{\perp} \cdot \operatorname{grad} u_{l}((z+\hat{z})(\tau))}{\left|(z+\hat{z})^{\prime}(\tau)\right|} d \tau$. 
Satz 8.5 Die Abbildungen

$$
\begin{aligned}
U \longrightarrow \mathcal{L}\left(C_{4 \pi}^{1, \alpha}(\mathbb{R}, \mathbb{R}), C_{4 \pi}^{0, \alpha}(\mathbb{R}, \mathbb{R})\right), & z \mapsto B_{2}(z), \\
U \longrightarrow \mathcal{L}\left(C_{4 \pi}^{0, \alpha}(\mathbb{R}, \mathbb{R}), C\left(\left[t_{a}, t_{e}\right], \mathbb{R}\right)\right), & z \mapsto \mathcal{T}_{0,1} \\
U \longrightarrow \mathcal{L}\left(C_{4 \pi}^{1, \alpha}(\mathbb{R}, \mathbb{R}), C\left(\left[t_{a}, t_{e}\right], \mathbb{R}\right)\right), & z \mapsto \mathcal{T}_{0,2}(z), \mathcal{T}_{0}(z)
\end{aligned}
$$

sind Fréchet-differenzierbar. Insbesondere gilt

$$
\mathcal{T}_{0}^{\prime}(z, h)=\mathcal{T}_{0,1}^{\prime}(z, h) \circ B_{2}(z)+\mathcal{T}_{0,1}(z) \circ B_{2}^{\prime}(z, h)+\mathcal{T}_{0,2}^{\prime}(z, h) .
$$

Beweis: Anwendung der Produktregel.

Im nächsten Satz wird für hinreichend glatte Dichten $\varphi$ der Zusammenhang von $\mathcal{T}_{0}^{\prime}(z, h) \varphi$ und $\frac{\partial}{\partial \nu} P^{\prime}(z, h) \varphi$ geklärt.

Satz 8.6 Seien $z \in U$ eine zulässige Parametrisierung und $\varphi \in C_{4 \pi}(\mathbb{R}, \mathbb{R})$ eine Dichte mit den zusätzlichen Eigenschaften $\left.\varphi\right|_{[0,2 \pi]},\left.\varphi\right|_{[2 \pi, 4 \pi]} \in C^{2, \alpha}$ sowie $\left.\varphi \circ z\right|_{[0,2 \pi]},\left.\varphi \circ z\right|_{[2 \pi, 4 \pi]} \in$ $S^{2,1+\delta}, 0<\delta<1$. Dann gilt

$$
\left(\frac{\partial}{\partial \nu} P^{\prime}(z, h) \varphi\right)((z+\hat{z})(t))=\frac{1}{2}\left(\mathcal{T}_{0}^{\prime}(z, h) \varphi\right)(t) \text { für } t_{a} \leq t \leq t_{e} .
$$

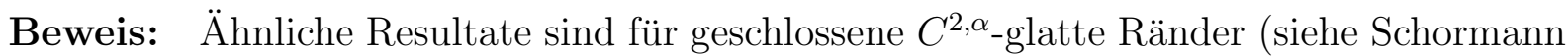
[5], Satz 4.16]) und Bögen der Klasse $C^{3}$ (siehe Mönch [44, Satz 2.5]) bekannt.

Sei $t \in\left[t_{a}, t_{e}\right]$ fest vorgegeben und $x=\hat{z}(t)$. Weiterhin seien $\Gamma_{1}, \Gamma_{2}$ und $\rho \in C^{3}(\Gamma)$ wie im Beweis von Satz 7.7 gewählt. Insbesondere lässt sich $\Gamma_{1}$ zu einer geschlossenen $C^{2, \alpha}$-glatten Kurve $\partial G_{z}$ fortsetzen.

Die Dichte $\varphi$ wird aufgespalten in

$$
\varphi=\rho \varphi+(1-\rho) \varphi
$$

Auf $\rho \varphi$ wird Satz 4.16 aus Schormann [5] angewendet. Demnach gilt für das Doppelschichtpotenzial $v$ zur Helmholtzgleichung über dem Rand $\partial G_{z} \in C^{2, \alpha}$ und Dichte $\psi \in C_{2 \pi}^{2, \alpha}(\mathbb{R}, \mathbb{C})$ die Sprungbeziehung

$$
\begin{aligned}
\frac{\partial v_{ \pm}^{\prime}}{\partial \nu}(z(t))= & \frac{1}{2}\left(\mathcal{T}_{k}^{\prime}(z, h) \psi\right)(t)-\operatorname{grad} v_{ \pm}(z(t)) \cdot \frac{\partial \nu}{\partial z}(z(t), h) \\
& -\sum_{i, j=1}^{2} \frac{\partial^{2} v_{ \pm}(z(t))}{\partial x_{i} \partial x_{j}} h_{i}(t) \nu_{j}(z(t)), 0 \leq t \leq 2 \pi .
\end{aligned}
$$


In dem hier vorliegenden Fall mit Dichte $\psi=\rho \varphi$ und $\hat{z}(t) \in \tilde{\Gamma}$ verschwinden wegen $z(t)=h(t)=(0,0), 2 \pi<t<4 \pi$ die letzten beiden Summanden in (8.13).

Desgleichen folgt aus [44, Satz 2.5], angewendet auf 3 Bogenstücke (und zwar den linken und rechten Rand von $\Gamma \backslash \Gamma_{2}$ sowie $\left.(z+\hat{z})(t), 0 \leq t \leq 2 \pi\right)$ das Resultat für die Dichte $(1-\rho) \varphi \in C^{1, \alpha, *}$.

Da sich die parametrisierte Randintegralgleichung (7.3) in Operatorform durch

$$
\left(I-A_{0}(z)\right) \varphi=-2 f
$$

schreiben lässt, sind die Lösung $u$ von (7.1) - (7.2) und deren Normalableitung auf $\tilde{\Gamma}$ gegeben durch die Funktionswerte der Abbildungen

$$
\begin{array}{ll}
\mathcal{P}: & U \subset X \longrightarrow C^{2}(D) \\
& z \longmapsto \mathcal{P}(z)=\left(P(z) \circ\left(I-A_{0}(z)\right)^{-1} \circ R(z)\right) f, \\
\mathcal{F}: & U \subset X \longrightarrow C(\tilde{\Gamma}) \\
& z \longmapsto \mathcal{F}(z)=\frac{1}{2}\left(\mathcal{T}_{0}(z) \circ\left(I-A_{0}(z)\right)^{-1} \circ R(z)\right) f .
\end{array}
$$

Nach der Produktregel sind $\mathcal{P}$ und $\mathcal{F}$ in $z \in U$ Fréchet-differenzierbar mit Ableitungen

$$
\begin{aligned}
\mathcal{F}^{\prime}(z, h) & =\frac{\partial}{\partial \nu} \mathcal{P}^{\prime}(z, h) \text { auf } \tilde{\Gamma} \\
\mathcal{P}^{\prime}(z, h) & =v_{1}+v_{2}+v_{3} \text { mit } \\
v_{1} & =\left(P^{\prime}(z, h)\left(I-A_{0}(z)\right)^{-1} R(z) f\right. \\
v_{2} & =P(z)\left(I-A_{0}(z)\right)^{-1} A_{0}^{\prime}(z, h)\left(I-A_{0}(z)\right)^{-1} R(z) f \\
v_{3} & =P(z)\left(I-A_{0}(z)\right)^{-1} R^{\prime}(z, h) f .
\end{aligned}
$$

Die Funktion $v_{3}$ ist identisch Null. Die Funktionen $v_{1}, v_{2}$ sind harmonisch in $D_{z+\hat{z}}$. Es sind die Randwerte von $v_{j}$ auf $\partial D_{z+\hat{z}}$ zu bestimmen.

Die Randwerte von $v_{2}$ sind wegen der Abbildungseigenschaft von $A_{0}^{\prime}(z, h)$ stetig und es gilt

$$
v_{2}((z+\hat{z})(t))=-\frac{1}{2}\left(A_{0}^{\prime}(z, h)\left(I-A_{0}(z)\right)^{-1} R(z) f\right)((z+\hat{z})(t)), 0 \leq t \leq 4 \pi .
$$

Für die Randwerte von $v_{1}$ gilt nach Satz 5.12 außerhalb der Ecken,

$$
v_{1}((z+\hat{z})(t))=\frac{1}{2}\left(A_{0}^{\prime}(z, h) \psi\right)(t)-\operatorname{grad}(P(z) \psi)((z+\hat{z})(t)) \cdot h(t), t \notin 2 \pi \mathbb{Z},
$$


wobei abkürzend $\psi=\left(I-A_{0}(z)\right)^{-1} R(z) f$ gesetzt wurde.

Durch Addition der drei Randbedingungen ergibt sich der nachfolgende Satz.

Satz 8.7 Die Fréchet-Ableitung $\mathcal{F}^{\prime}(z, h)$ des Randwerteoperators ist gegeben durch die Normalableitung $\frac{\partial}{\partial \nu} v$ auf $\tilde{\Gamma}$, wobei $v$ das zum Gebiet $\partial D_{z+\hat{z}}$ gehörige innere Dirichletproblem

$$
\begin{aligned}
\triangle v & =0 \quad \text { in } D_{z+\hat{z}} \\
v((z+\hat{z})(t)) & =-\left(\operatorname{grad} P(z)\left(I-A_{0}(z)\right)^{-1} R(z) f\right)_{-}((z+\hat{z})(t)) \cdot h(t), t \notin 2 \pi \mathbb{Z},
\end{aligned}
$$

löst derart, dass $v-v_{1}$ stetig bis in den Rand ist.

Für die stetigen Randwerte von $v_{2}$ ist das innere Dirichletproblem zu lösen und mit dem Vorwärtslöser ist die Normalableitung auf dem Rand zu ermitteln. Hierfür ist das Implementieren von $A_{0}^{\prime}(z, h)$ erforderlich. Aus Satz 8.2 wird deutlich, dass $A_{0}^{\prime}(z, h)$ eine geeignete Linearkombination von Einfach- und Doppelschichtpotenzialen sowie deren Fréchet-Ableitungen ist.

Für die Berechnung der Normalableitung von $v_{1}$ ist der Operator $\mathcal{T}_{0}^{\prime}(z, h)$ zu implementieren. Auch hierbei treten neben $\mathcal{T}_{0}$ nur stetige oder schwach singuläre Kerne auf.

\subsection{Numerische Ergebnisse beim inversen Impedanz- randwertproblem}

Seien $f \in C_{0}^{2, \alpha}(\Gamma)$ und $\frac{\partial u}{\partial \nu}^{\text {meas }}$ die zugehörigen Messwerte für die exakten Neumannrandwerte gemäß Definition 7.2. Die Gleichung

$$
\mathcal{F}(z)=\frac{\partial u}{\partial \nu}^{\text {meas }}
$$

ist zu lösen. (Zur Erinnerung: Bei der Abbildung $z \mapsto \mathcal{F}(z)$ wird das direkte Problem bezüglich des Randes $z+\hat{z}$ gerechnet.) Dabei liegen die Messwerte $\frac{\partial u}{\partial \nu}^{\text {meas }}$ nur an diskreten Stellen $\hat{x}_{1}, \ldots, \hat{x}_{M} \in \tilde{\Gamma}$ vor.

Analog zu der im Kapitel 6 beschriebenen Linearisierung, Wahl eines endlichdimensionalen Teilraumes $Q_{N}=\operatorname{span}\left\{q_{1}, \ldots, q_{N}\right\} \subset U$ mit $N<M$ und einer Regularisierung (ohne Bestrafung höherer Ableitungen) führt dies zu einem Iterationsverfahren

$$
z_{p+1}=z_{p}+h_{p}, p=0,1,2, \ldots
$$


wobei $h_{p}$ gegeben ist durch

$$
h_{p}=\sum_{j=1}^{N} a_{j}^{(p)} q_{j} \in Q_{N}
$$

mit den reellen Koeffizienten $a_{j}^{(p)}$, die ein Gleichungssystem der Form

$$
\left(\alpha_{p} I+C^{(p)^{t}} C^{(p)}\right) a^{(p)}=C^{(p)^{t}} g^{(p)}
$$

erfüllen.

Die Komponenten der Matrix $C^{(p)} \in \mathbb{R}^{M \times N}$ und die der Vektoren $a^{(p)} \in \mathbb{R}^{N}, g^{(p)} \in \mathbb{R}^{M}$ sind gegeben durch

$$
\begin{aligned}
c_{i j}^{(p)} & =\mathcal{F}^{\prime}\left(z_{p}, q_{j}\right)\left(\hat{x}_{i}\right), i=1, \ldots, M, j=1, \ldots, N, \\
g_{i}^{(p)} & =\frac{\partial u^{\text {meas }}}{\partial \nu}\left(\hat{x}_{i}\right)-\mathcal{F}\left(z_{p}\right)\left(\hat{x}_{i}\right), i=1, \ldots, M, \\
g^{(p)} & =\left(g_{i}^{(p)}\right)_{1 \leq i \leq M}, \\
a^{(p)} & =\left(a_{j}^{(p)}\right)_{1 \leq j \leq N} .
\end{aligned}
$$

Die Basiselemente für den Approximationsraum $Q_{N}, N \geq 3$, seien durch

$$
q_{j}(t)=-r_{j}(t)\left(\begin{array}{c}
\cos (t / 2) \\
\sin (t / 2)
\end{array}\right), j=1, \ldots, N, 0 \leq t \leq 2 \pi
$$

mit den Radialanteilen

$$
r_{1}(t)=t(2 \pi-t)^{2}, \quad r_{2}(t)=t^{2}(2 \pi-t)
$$

und

$$
r_{j}(t)=\sin \left(\frac{(j-2) t}{2}\right), j=3, \ldots, N
$$

gegeben. Die Startkurven $z_{0} \in U$ und $\hat{z}$ sind so zu wählen, dass sie den Anforderungen aus Abschnitt 8.2 genügen. Insbesondere sind $\hat{z}(0)=x^{(0)}, \hat{z}(2 \pi)=x^{(1)}$ und die Negativität der zweiten Komponente von $\hat{z}(t)$ auf $0<t<2 \pi$ zu erfüllen.

Für $p \geq 1$ hat die Parametrisierung des unteren Randes die Form

$$
z_{p}(t)=z_{0}(t)+\sum_{j=1}^{N} \tilde{a}_{j}^{(p)} r_{j}(t)\left(\begin{array}{c}
\cos (t / 2) \\
\sin (t / 2)
\end{array}\right), 0 \leq t \leq 2 \pi, \tilde{a}_{j}^{(p)}=-\sum_{l=0}^{p-1} a_{j}^{(l)} .
$$


Differenzieren führt zu

$$
z_{p}^{\prime}(t)=z_{0}^{\prime}(t)+\sum_{j=1}^{N} \tilde{a}_{j}^{(p)} r_{j}^{\prime}(t)\left(\begin{array}{r}
\cos (t / 2) \\
\sin (t / 2)
\end{array}\right)+\sum_{j=1}^{N} \tilde{a}_{j}^{(p)} r_{j}(t) \frac{1}{2}\left(\begin{array}{r}
-\sin (t / 2) \\
\cos (t / 2)
\end{array}\right) .
$$

Aus der letzten Zeile sind die Werte $z_{p}^{\prime}(0)$ und $z_{p}^{\prime}(2 \pi)$ ablesbar. Insbesondere wird deutlich, dass neben $z_{0}+\hat{z}$ ausschließlich $q_{1}$ den Innenwinkel bei $x^{(0)}$ und $q_{2}$ den Innenwinkel bei $x^{(1)}$ kontrollieren.

In den numerischen Beispielen sind die Ecken stets durch $x^{(0)}=(-1,0)$ und $x^{(1)}=(1,0)$ gegeben und als Startkurven werden

$$
z_{0}(t)=(0,0), \hat{z}(t)=-\left(\begin{array}{c}
\cos (t / 2) \\
\sin (t / 2)
\end{array}\right), 0 \leq t \leq 2 \pi
$$

gewählt.

Es wird versucht, nachfolgende aus Abschnitt 7.4 bekannte Kurven zu rekonstruieren:

$$
\begin{aligned}
& z_{2}^{(\gamma)}(t)=\frac{0.5(t-2 \pi)-1}{1+\frac{\pi}{2}}(\cos (t / 2), 3 \sin (t / 2))+\left(\frac{\frac{\pi}{2}}{1+\frac{\pi}{2}}, 0\right) \\
& z_{4}^{(\gamma)}(t)=-\left(1-\frac{1}{3} \sin (t / 2)+\frac{1}{6} \sin (1.5 t)\right)(\cos (t / 2), 4 \sin (t / 2)) \\
& z_{6}^{(\gamma)}(t)=\left(\frac{1}{\pi}(t-\pi),-3 \sin (t / 2)\right), 0 \leq t \leq 2 \pi
\end{aligned}
$$

Durch die Wahl von unterschiedlichen Skalierungsfaktoren vor $\cos (t / 2)$ und $\sin (t / 2)$ ist sichergestellt, dass die zu rekonstruierenden Kurven nicht im Ansatzraum vorkommen.

Beispiel 8.8 Der obere Rand $\Gamma$ wird gegeben durch die halbe Erdnuss

$$
z_{4}^{(\Gamma)}(t)=-\left(1+\frac{1}{3} \sin (t / 2)-\frac{1}{6} \sin (1.5 t)\right)(\cos (t / 2), 4 \sin (t / 2)), 2 \pi \leq t \leq 4 \pi .
$$

Die Randwerte $f$ auf $\Gamma$ lauten $f(z(t))=|z(t)|^{2}-1,2 \pi \leq t \leq 4 \pi$.

Beispiel 8.9 Der obere Rand $\Gamma$ wird gegeben durch

$$
z_{5}^{(\Gamma)}(t)=-(1-\sin (t / 2))(\cos (t / 2), 2 \sin (t / 2)), 2 \pi \leq t \leq 4 \pi .
$$


Die vorgegebenen Randwerte $f$ lauten in Polarkoordinaten $(r, \vartheta) \in \mathbb{R}^{+} \times[-\pi, \pi)$ (mit dem Koordinatenursprung in $x^{(0)}$ )

$$
f(r, \vartheta)=r^{3.5} \sin (3.5 \vartheta),(r, \vartheta) \in \Gamma .
$$

Die synthetischen Daten wurden mit der Substitution nach Korobov mit Grad $m=4$ und der Stützstellenzahl $4 n=4 \cdot 32$ erzeugt. Die Messpunkte $\hat{x}_{1}, \ldots, \hat{x}_{M}$ stammen aus dem Beobachtungsbereich $z^{(\Gamma)}(t), \frac{5 \pi}{2} \leq t \leq \frac{7 \pi}{2}$, das heißt

$$
\hat{x}_{i}=z^{(\Gamma)}\left(\frac{5 \pi}{2}+\frac{(i-1) \pi}{M-1}\right), i=1, \ldots, M .
$$

Beim inversen Löser wurden zur Vermeidung von Inverse Crimes mit anderer Stützstellenzahl und einer anderen Substitution (nach Kreß mit $m=5$ ) gerechnet. Die jeweiligen Parameter wie Anzahl der Iterationsschritte $p$, Anzahl der Stützstellen 4n, die Dimension des Ansatzraums $N$, Anzahl der Messpunkte $M$, der Regularisierungsparameter $\alpha_{p}$ im $p$-ten Newton-Schritt und das Residuum sind den Legenden zu entnehmen.

Für die Beschreibung eines Iterationsschrittes zum Newtonverfahren (einschließlich des Abbruchkriteriums) wird auf Abschnitt 6.1 verwiesen. Es sind wiederum in jedem Schritt $N+1$ direkte Probleme zu lösen.

Der rekonstruierte Teil ist mit einer durchgehenden, das Originalgebiet mit einer gestrichelten Linie dargestellt.

Der Algorithmus liefert gute Ergebnisse, obwohl in der Regel die Größen der Innenwinkel nicht rekonstruiert werden.

Es ist denkbar, dass sich bei Modifizierung des Strafterms im Tikhonov-Funktional durch Berücksichtigung höherer Ableitungen oder Verwendung von mehr Messreihen (mehrere direkte Probleme) die Rekonstruktionen weiter verbessern lassen. 


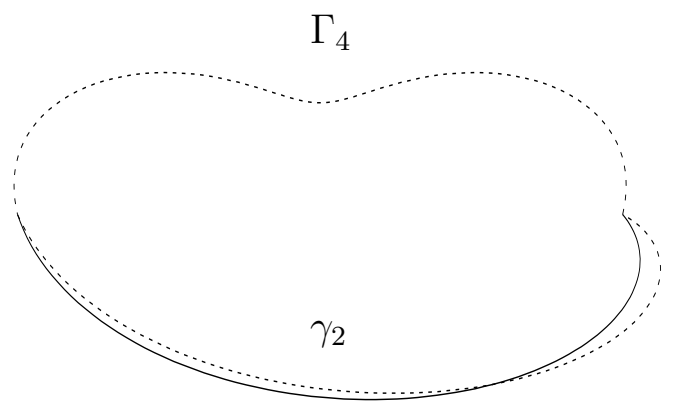

$$
\begin{aligned}
p_{\text {end }} & =42, \\
n & =36, \\
N & =15, \\
M & =32, \\
\alpha_{p} & =1.0 \cdot 0.8^{p}, \\
\text { Res } & =3.6 \cdot 10^{-4},
\end{aligned}
$$

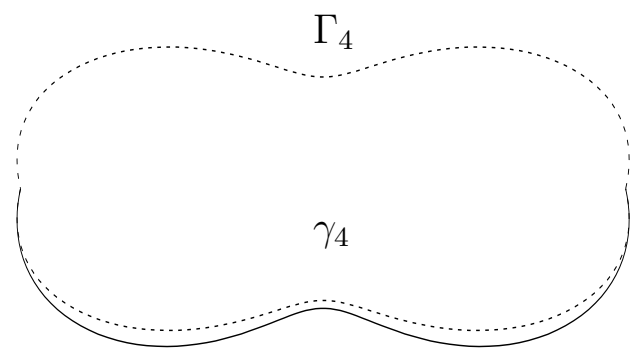

$$
\begin{aligned}
p_{\text {end }} & =46, \\
n & =36, \\
N & =15, \\
M & =32, \\
\alpha_{p} & =1.0 \cdot 0.8^{p}, \\
\text { Res } & =2.1 \cdot 10^{-6},
\end{aligned}
$$

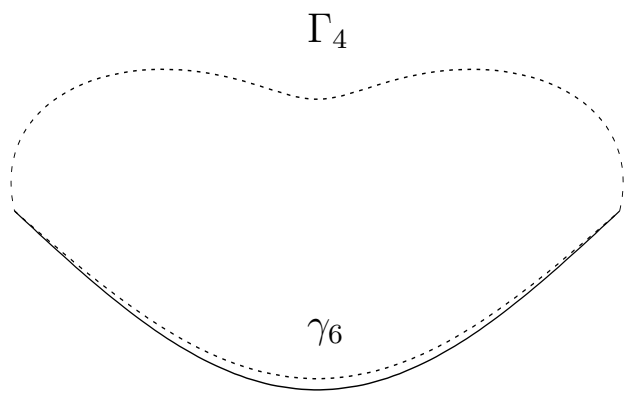

$$
\begin{aligned}
p_{\text {end }} & =51, \\
n & =36 \\
N & =15 \\
M & =32 \\
\alpha_{p} & =1.0 \cdot 0.85^{p}, \\
\text { Res } & =3.8 \cdot 10^{-6}
\end{aligned}
$$

Rekonstruktionen aus Beispiel 8.8 


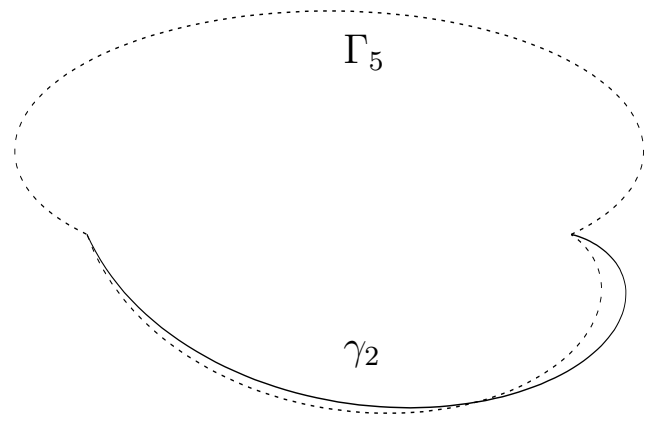

$$
\begin{aligned}
p_{\text {end }} & =51, \\
n & =48, \\
N & =18, \\
M & =32, \\
\alpha_{p} & =1.0 \cdot 0.75^{p}, \\
\text { Res } & =4.1 \cdot 10^{-3},
\end{aligned}
$$

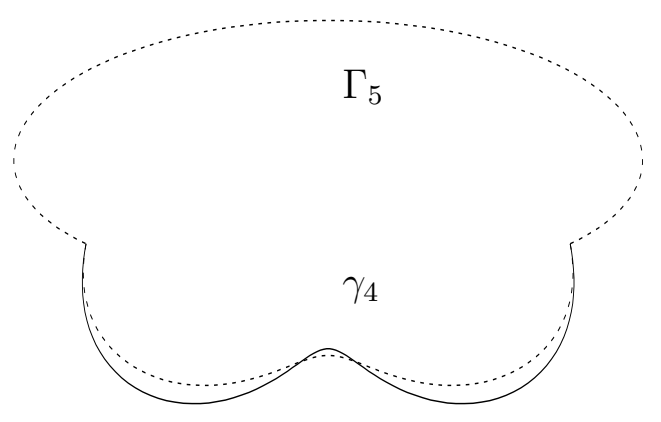

$$
\begin{aligned}
p_{\text {end }} & =46, \\
n & =48 \\
N & =18 \\
M & =32 \\
\alpha_{p} & =1.0 \cdot 0.8^{p} \\
\text { Res } & =1.1 \cdot 10^{-5}
\end{aligned}
$$

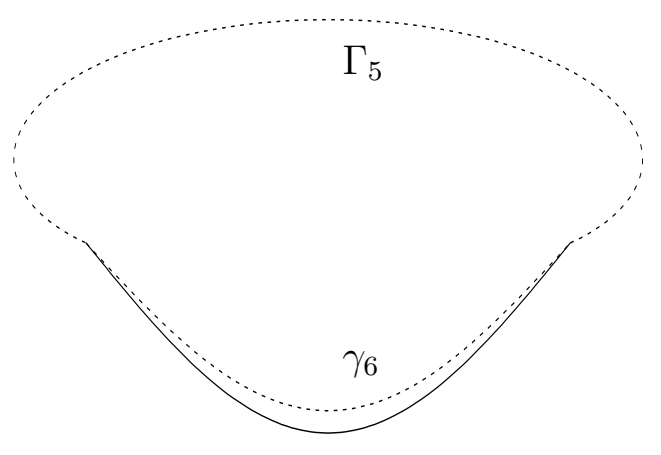

$$
\begin{aligned}
p_{\text {end }} & =61, \\
n & =48 \\
N & =18 \\
M & =32 \\
\alpha_{p} & =1.0 \cdot 0.85^{p}, \\
\text { Res } & =7.2 \cdot 10^{-5},
\end{aligned}
$$

Rekonstruktionen aus Beispiel 8.9 


\section{Literaturverzeichnis}

[1] R.Adams, Sobolev Spaces, Academic Press, New York, San Francisco, London, 1975.

[2] F.Boeckh, Die numerische Behandlung eines Randwertproblems mit einer Integralgleichungsmethode bei einem unendlich ausgedehnten Rand mit Ecken, Diplomarbeit, Institut für Numerische und Angewandte Mathematik Universität Göttingen, Göttingen, 1988.

[3] A.L.Bukhgeim, J.Cheng and M.Yamamoto, Stability for an inverse boundary problem of determining a part of boundary, UTMS 98-32, University of Tokyo, Graduate school of mathematical sciences, Komaba, Tokyo, 1998.

[4] A.L.Bukhgeim, J.Cheng and M.Yamamoto, Conditional stability in an inverse problem of determining non-smooth boundary, UTMS 98-36, University of Tokyo, Graduate school of mathematical sciences, Komaba, Tokyo, 1998.

[5] R.Chapko and R.Kress, On a quadrature method for a logarithmic integral equation of the first kind, Contributions in Numerical Mathematics, World Scientific Series in Applicable Analysis 2, World Scientific, Singapore, 1993.

[6] D.Colton and R.Kress, Integral Equation Methods in Scattering Theory, WileyInterscience Publication, New York, 1983.

[7] D.Colton and R.Kress, Inverse Acoustic and Electromagnetic Scattering Theory, Springer-Verlag, Berlin, Heidelberg, New York, 1992.

[8] M.Costabel, M.Dauge, S.Nicaise, Boundary value problems and integral equations in nonsmooth domains, Lecture notes in pure and applied mathematics 167, Marcel Dekker inc., New York, Basel, Hong Kong, 1995.

[9] R.Courant and D.Hilbert, Methods of Mathematical Physics, Volume 1, Interscience Publishers, New York, 1966. 
[10] M.Dauge, Elliptic Boundary Value Problems on Corner Domains, Lecture Notes in Mathematics 1341, Springer-Verlag, Berlin, Heidelberg, 1988.

[11] P.Deuflhard, H.P.Engl and O.Scherzer, A convergence analysis of iterative methods for the solution of nonlinear ill-posed problems under affinely invariant conditions, Inverse Problems 14, 1081-1106, (1998).

[12] J.Elschner and E.P.Stephan, A discrete collocation method for Symm's integral equation on curves with corners, Journal of Computational and Applied Mathematics 75, 131-146, (1996).

[13] J.Elschner, Y.Jeon, I.H.Sloan and E.P.Stephan, Discrete qualocation methods for logarithmic-kernel integral equations on a piecewise smooth boundary, Advances in Computational Mathematics 7, 547-571, (1997).

[14] T.Gerlach, Die Streuung zeitharmonischer elektromagnetischer Wellen an chiralen Medien, Dissertation, Institut für Numerische und Angewandte Mathematik Universität Göttingen, Göttingen, 1999.

[15] P.Grisvard, Elliptic Problems in Nonsmooth Domains, Pitman Advanced Publishing Program, Boston-London-Melbourne, 1985.

[16] P.Grisvard, Singularities in Boundary Value Problems, Springer-Verlag, BerlinHeidelberg-New York, 1992.

[17] C.W.Groetsch, Inverse Problems in the Mathematical Sciences, Vieweg, Braunschweig, 1993.

[18] W.Hackbusch, Integralgleichungen: Theorie und Numerik, Teubner, Stuttgart, 1989.

[19] R.Hartke, Direktes und Inverses Dirichletsches Randwertproblem zur Helmholtzgleichung in einer lokal gestörten Halbebene, Diplomarbeit, Institut für Numerische und Angewandte Mathematik Universität Göttingen, Göttingen, 1993.

[20] E.Heinz, Vorlesung zu Differential- und Integralrechnung III, Wintersemester 1986/87 Mathematisches Institut Universität Göttingen, Göttingen, 1987.

[21] T.Hohage, Newton-Verfahren beim inversen Neumann-Problem zur Helmholtzgleichung, Diplomarbeit, Institut für Numerische und Angewandte Mathematik Universität Göttingen, Göttingen, 1996. 
[22] T.Hohage and C.Schormann, A Newton-type method for a transmission problem in inverse scattering, Inverse Problems 14, 1207-1227, (1998).

[23] O.D.Kellogg, Foundations of Potential Theory, Springer-Verlag, Berlin, Heidelberg, New York, 1929.

[24] J.K.Knowles and T.A.Pucik, Uniqueness for plane crack problems in linear elastostatics, Journal of Elasticity 3, 155-160, (1973).

[25] H.König, Jahresbericht der DMV 66, Teubner, Stuttgart, 1964, 119-138.

[26] R.Kress, Über die numerische Berechnung konjugierter Funktionen, Computing 10, 177-187, (1972).

[27] R.Kress, A boundary integral equation method for a Neumann boundary problem for force - free fields, Journal of Engineering Mathematics 15, 29-48, (1981).

[28] R.Kress, A Nyström method for boundary integral equations in domains with cornerns, Numerische Mathematik 58, 145-161, (1990).

[29] R.Kress and I.H.Sloan, On the numerical solution of a logarithmic integral equation of the first kind for the Helholtzequation, Numerische Mathematik 66, 199-214, (1993).

[30] R.Kress, A Newton method in inverse obstacle scattering, In: Inverse Problems in Engineering Mechanics, (Bui et al, eds) 425-432, Balkema, Rotterdam, 1994.

[31] R.Kress, Inverse scattering from an open arc, Mathematical Methods in the Applied Science 18, 267-293, (1995).

[32] R.Kress, Fréchet differentiability of the far field operator for scattering from a crack, Journal on Inverse and Ill-Posed Problems 3, 305-313, (1995).

[33] R.Kress, On the numerical solution of a hypersingular integral equation in scattering theory, Journal of Computational and Applied Mathematics 61, 345-360, (1995).

[34] R.Kress, I.H.Sloan and F.Stenger, A sinc quadrature method for the doublelayer integral equation in planar domains with corners, Journal of Integral Equations and Applications 10, 291-317, (1998).

[35] R.Kress, Linear Integral Equations, Springer-Verlag, Berlin, Heidelberg, New York, Second Edition, 1999. 
[36] R.Kress, On the low wave number behavior of two-dimensional scattering problems for an open arc, Journal for Analysis and its Applications 18, 297-305, (1999).

[37] R.Kress and T.Tran Inverse scattering for a locally perturbed half-plane, Inverse Problems 16, 1541-1559, (2000).

[38] R.Kussmaul, Ein numerisches Verfahren zur Lösung des Neumannschen Aussenraumproblems für die Helmholtzsche Schwingungsgleichung, Computing 4, 246-273, (1969).

[39] P.Kümpel, Das Nyström Verfahren für das Dirichlet Problem zur Helmholtzgleichung in zweidimensionalen Gebieten mit Ecken, Diplomarbeit, Institut für Numerische und Angewandte Mathematik Universität Göttingen, Göttingen, 1997.

[40] R.Leis, Zur Dirichletschen Randwertaufgabe des Außenraums der Schwingungsgleichung, Mathematische Zeitschrift 90, 205-211, (1965).

[41] P.A.Lewis and G.R.Wickham, The diffraction of $S H$ waves by an arbitrary shaped crack in two dimensions, Proceedings of the Royal Society of London 340, 503-529, (1992).

[42] E.Martensen, Über eine Methode zum räumlichen Neumannschen Problem mit einer Anwendung für torusartige Berandungen., Acta Math. 109, 75-135, (1963).

[43] L.Mönch Ein Quadraturformelverfahren für eine hypersinguläre Integralgleichung bei der Helmholtzgleichung im $\mathbb{R}^{2}$, Diplomarbeit, Institut für Numerische und Angewandte Mathematik Universität Göttingen, Göttingen, 1994.

[44] L.Mönch Die numerische Lösung des inversen akustischen Streuproblems am schallharten Kurvenbogen, Dissertation, Institut für Numerische und Angewandte Mathematik Universität Göttingen, Göttingen, 1994.

[45] R.Potthast, Fréchet differentiability of boundary integral operators in inverse acoustic scattering, Inverse Problems 10, 431-447, (1994).

[46] R.Potthast, Fréchet Differenzierbarkeit von Randintegraloperatoren und Randwertproblemen zur Helmholtzgleichung und den zeitharmonischen Maxwellgleichungen, Dissertation, Institut für Numerische und Angewandte Mathematik Universität Göttingen, Göttingen, 1994.

[47] R.Potthast, Domain Derivatives in Electromagnetic Scattering, Mathematical Methods in the Applied Sciences 19, 1157-1175, (1996). 
[48] A.G.Ramm, New method for proving uniqueness theorems for obstacle inverse scattering problems, Applied Mathematics Letters 6, 19-21, (1993).

[49] J.Rauch, Partial Differential Equations, Springer-Verlag, New York, 1991.

[50] C.Ruland, Ein Verfahren zur Lösung von $\left(\triangle+k^{2}\right) u=0$ in Außengebieten mit Ecken, Applicable Analysis 7, 69-79, (1978).

[51] C.Schormann, Newton-Verfahren für ein inverses Transmissionsproblem bei elektromagnetischen Wellen, Diplomarbeit, Institut für Numerische und Angewandte Mathematik Universität Göttingen, Göttingen, 1996.

[52] C.Schormann, Analytische und numerische Untersuchungen bei inversen Transmissionsproblemen zur zeitharmonischen Wellengleichung, Dissertation, Institut für Numerische und Angewandte Mathematik Universität Göttingen, Göttingen, 2000.

[53] A.Sidi, A new variable transformation for numerical integration, In: Numerical Integration IV, International Series of Numerical Mathematics, Birkhäuser Verlag Basel 112, (1993), 359-373.

[54] I.H.Sloan, Analysis of general quadrature methods for integral equations of the second kind, Numerische Mathematik 38, 263-278, (1981).

[55] A.Vogt, Substitutionsmethoden beim Nyström - Verfahren für Integralgleichungen zweiter Art, Diplomarbeit, Institut für Numerische und Angewandte Mathematik Universität Göttingen, Göttingen, 1997.

[56] G.R.Wickham, The diffraction of stress waves by a plane finite crack in two dimensions: uniqueness and existence, Proceedings of the Royal Society of London 378, 241-261, (1981).

[57] A.Willers, Integralgleichungsmethoden bei der Reflexion an lokal gestörten Ebenen, Dissertation, Institut für Numerische und Angewandte Mathematik Universität Göttingen, Göttingen, 1985. 



\section{Lebenslauf}

persönliche Daten

Name

Andreas Vogt

Geburtsdatum

28.06.1972

Geburtsort

Leinefelde

Staatsangehörigkeit

deutsch

Eltern

Erich Vogt, Bäckermeister

Ingeburg Vogt, geb. Liepert, Bürokauffrau

E-Mail

avogt@math.uni-goettingen.de

Ausbildung

$09.1979-08.1989 \quad$ Polytechnische Oberschule Worbis

09.1989 - $06.1991 \quad$ Spezialklassen für Mathematik und Physik an der Martin-Luther-Universität Halle-Wittenberg

Juni 1991 Abschluss mit dem Abitur

WS 91/92 - SS 1997

Studium der Mathematik mit Nebenfach Physik an der Georg-August-Universität in Göttingen

Mai 1997

Abschluss mit dem Diplom

$09.1997-06.1998 \quad$ Wehrdienst

seit WS 98/99

Promotionsstudium in Mathematik

Tätigkeiten

WS 93/94, SS 94, wissenschaftliche Hilfskraft am Institut für Numerische SS 95, WS 95/96 und Angewandte Mathematik der Universität Göttingen

$06.1997-08.1997$ und wissenschaftlicher Mitarbeiter am Institut für Numerische seit 07.1998 und Angewandte Mathematik der Universität Göttingen 